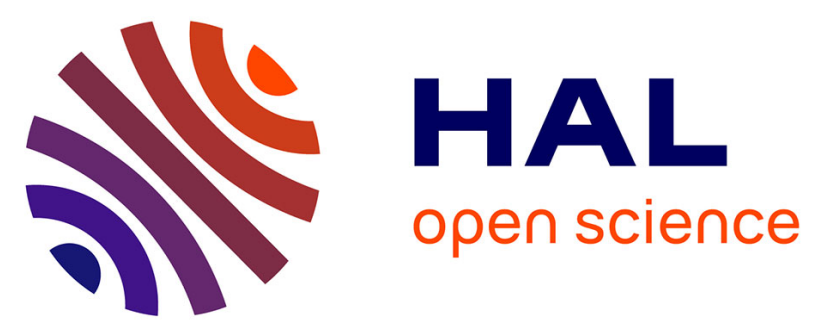

\title{
Isoprostanes, neuroprostanes and phytoprostanes: An overview of 25 years of research in chemistry and biology
}

Jean-Marie Galano, Yiu Yiu Lee, Camille Oger, Claire Vigor, Joseph Vercauteren, Thierry Durand, Martin Giera, Jetty Chung-Yung Lee

\section{- To cite this version:}

Jean-Marie Galano, Yiu Yiu Lee, Camille Oger, Claire Vigor, Joseph Vercauteren, et al.. Isoprostanes, neuroprostanes and phytoprostanes: An overview of 25 years of research in chemistry and biology. Progress in Lipid Research, 2017, 68, pp.83-108. 10.1016/j.plipres.2017.09.004 . hal-02594327

\section{HAL Id: hal-02594327 \\ https://hal.science/hal-02594327}

Submitted on 15 May 2020

HAL is a multi-disciplinary open access archive for the deposit and dissemination of scientific research documents, whether they are published or not. The documents may come from teaching and research institutions in France or abroad, or from public or private research centers.
L'archive ouverte pluridisciplinaire HAL, est destinée au dépôt et à la diffusion de documents scientifiques de niveau recherche, publiés ou non, émanant des établissements d'enseignement et de recherche français ou étrangers, des laboratoires publics ou privés. 


\title{
Isoprostanes, neuroprostanes and phytoprostanes: An overview of 25 years of research in chemistry and biology
}

\author{
Jean-Marie Galano ${ }^{\mathrm{a}}$, Yiu Yiu Lee ${ }^{\mathrm{b}}$, Camille Oger ${ }^{\mathrm{a}}$, Claire Vigor ${ }^{\mathrm{a}}$, Joseph Vercauteren ${ }^{\mathrm{a}}$, \\ Thierry Durand ${ }^{\mathrm{a}}$, Martin Giera ${ }^{\mathrm{c}}$, Jetty Chung-Yung Lee ${ }^{\mathrm{b}, *}$ \\ a Institut des Biomolécules Max Mousseron, UMR 5247 CNRS, ENSCM, Université de Montpellier, France \\ b School of Biological Sciences, The University of Hong Kong, Hong Kong Special Administrative Region \\ ${ }^{\mathrm{c}}$ Leiden University Medical Center, Center for Proteomics and Metabolomics, Albinusdreef 2, 2300RC Leiden, The Netherlands
}

\begin{abstract}
A B S T R A C T
Since the beginning of the 1990's diverse types of metabolites originating from polyunsaturated fatty acids, formed under autooxidative conditions were discovered. Known as prostaglandin isomers (or isoprostanoids) originating from arachidonic acid, neuroprostanes from docosahexaenoic acid, and phytoprostanes from $\alpha$-linolenic acid proved to be prevalent in biology. The syntheses of these compounds by organic chemists and the development of sophisticated mass spectrometry methods has boosted our understanding of the isoprostanoid biology. In recent years, it has become accepted that these molecules not only serve as markers of oxidative damage but also exhibit a wide range of bioactivities. In addition, isoprostanoids have emerged as indicators of oxidative stress in humans and their environment. This review explores in detail the isoprostanoid chemistry and biology that has been achieved in the past three decades.
\end{abstract}

\section{Introduction}

The discovery of isoprostanes (IsoPs) nearly three decades ago as biomarker of oxidative stress [1] opened a new field of research in arachidonic acid (AA) and its eicosanoid metabolism. Today, it is accepted that IsoPs are more than only biomarkers and bystanders of physiological processes. IsoPs have been shown to act as lipid mediators in vasoconstriction and platelet aggregation, and may participate in intracellular signaling through the activation of prostanoid receptors [2]. Unlike the prostaglandins (PGs), IsoP biosynthesis is not dependent on the cyclooxygenase enzyme, instead it is propagated by free radical reactions, mainly driven by reactive oxygen species (ROS). Presently, it is well accepted that IsoP generation takes place in situ by the reaction of ROS with glycerophospholipids, mainly phosphatidylcholine. The esterified IsoPs can be released from the phospholipid by the action of phospholipase $\mathrm{A}_{2}$ and platelet activating factor acetylhydrolase. While a plethora of isomeric IsoPs have been described, undoubtedly $15-\mathrm{F}_{2 \mathrm{t}^{-}}$ Isoprostane $\left(15-\mathrm{F}_{2 \mathrm{t}}\right.$-IsoP) is the predominant one investigated. Once released into the circulation, the free $15-\mathrm{F}_{2 \mathrm{t}}$-IsoP is $\beta$-oxidized to form an intermediate compound, 2,3-dinor- $15-\mathrm{F}_{2 \mathrm{t}}$-IsoP that can further undergo reduction to 2,3-dinor-5,6-dihydro- $15-\mathrm{F}_{2 \mathrm{t}}$-IsoP for urinary excretion [3]. However, an alternative $\beta$-oxidation processes takes place in rodents to produce 2,3,4,5-tetranor- $15-\mathrm{F}_{2 \mathrm{t}}$-IsoP (Section 2.2) $[4,5]$.

Unlike the IsoPs, the metabolism of neuroprostanes (NeuroPs) and phytoprostanes (PhytoPs) originating from docosahexaenoic acid (DHA) and $\alpha$-linolenic acid (ALA) are not well elucidated. While the formation of NeuroPs and PhytoPs follows the same pathways as the IsoPs, their metabolism and excretion are yet poorly explored. Nevertheless, the importance of NeuroPs and PhytoPs as significant lipid mediators has clearly been established only in recent years [6-9].

In turn, understanding both, the chemistry and biology of the isoprostanoids (IsoPs, NeuroPs and PhytoPs) are necessary to improve the

Abbreviations: AA, Arachidonic acid; AD, Alzheimer's disease; AdA, Adrenic acid; ALA, $\alpha$-Linolenic acid; APCI, Atmospheric pressure chemical ionization; BHT, Butylated hydro-

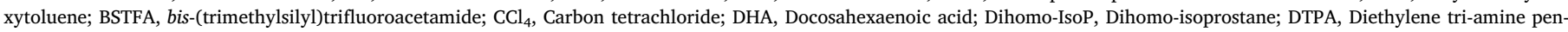

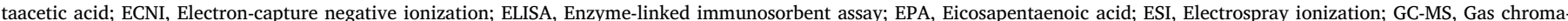

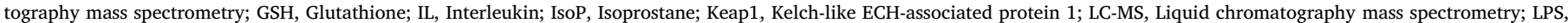

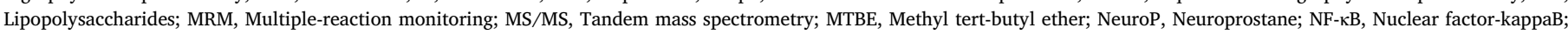

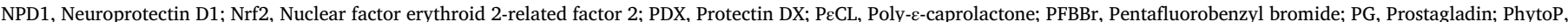

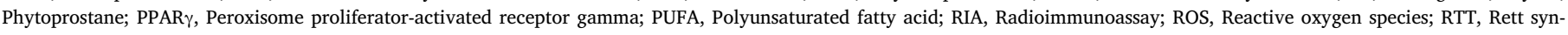
drome; SIM, Selected-ion monitoring; SPE, Solid phase extraction; TLC, Thin layer chromatography; TPP, Triphenylphosphine; TPR, Thromboxane receptor

* Corresponding author at: School of Biological Sciences, The University of Hong Kong, Pokfulam Road, Hong Kong Special Administrative Region.

E-mail address: jettylee@hku.hk (J.C.-Y. Lee). 


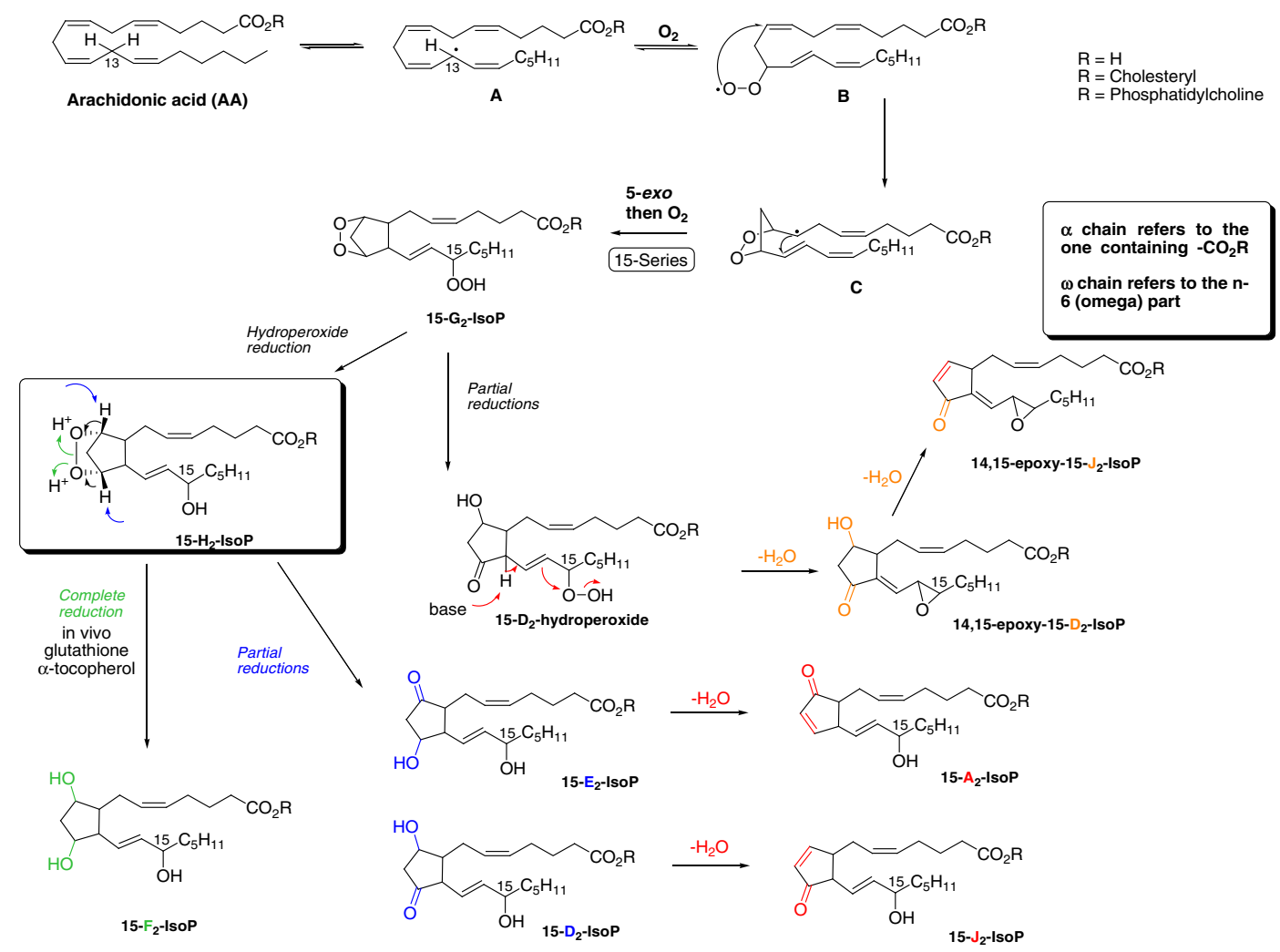

Scheme 1. Biosynthesis of isoprostanoids type A, D, E, F and J and epoxy-isomers.

analytical approaches in order to understand the metabolism and biology of isoprostanoids. In this review, we present a concise overview of the current knowledge concerning the chemistry and biology of IsoPs, NeuroPs and PhytoPs and hope that our compendium will help others to further explore the biological relevance of isoprostanoids.

\section{Understanding isoprostanoids in vivo formation}

The word isoprostanoid refers to isomers of PGs. In fact, the lateral chains bearing by the cyclopentane ring are in 1,2-trans relationship (spatially opposed) for the PGs as they are in 1,2-cis relationship (in the same plane) for the isoprostanes (IsoPs). The biosynthesis of PGs is well established since the 1970s and involves enzymatic COX-mediated mechanisms. However, the formation of IsoPs was uncovered in the 1990s through publication in the field of PGs [1]. Morrow and coworkers showed that arachidonic acid (AA, C20:4 n-6) exposed to carbon tetrachloride $\left(\mathrm{CCl}_{4}\right)$ was oxidized in vivo to racemic IsoPs and racemic PGs, independent from any enzymatic COX action.

The biosynthesis of these metabolites, described in Scheme 1 is now well-established and proceeds through several radical steps $[1,10]$. Polyunsaturated fatty acids (PUFA) such as AA are composed of bisallylic structures; double bonds separated by a methylene group $\left(-\mathrm{CH}=\mathrm{CH}-\mathrm{CH}_{2}-\mathrm{CH}=\mathrm{CH}-\right.$ ), where $\alpha$-hydrogen atoms are particularly easy to remove by the actions of free radicals, supported by the mesomeric stabilization of the resulting radical (Scheme 1). Physiologically, after oxidative stress injury and the release of free radicals in the cellular membranes, one hydrogen atom is abstracted from AA (intermediate A). The resulting carbon radical, in the presence of oxygen, further proceeds to form a peroxyl radical (compound B) and eventually the formation of an endoperoxide carbon centred radical (compound $\mathrm{C}$ ). This intermediate then proceeds through a cyclization step to form the cyclopentane ring. The second consecutive 5-exo-trig cyclization follows the radical chemistry rules (Baldwin rules [11,12]) and is the determinant step going along with the main PGs and IsoPs difference (e. g. lateral chains relationships), as it predominantly leads to a cis configuration of the lateral chains along with the trans configuration (PG isomers). The generation of the cyclopentane ring results in the formation of another radical which reacts with molecular oxygen to produce an endoperoxide-hydroperoxide intermediate after final $\mathrm{H}$ atom transfer, also known as $\mathrm{G}_{2}$-IsoP. Prior to the endoperoxide reduction, the hydroperoxide is first reduced to form the $\mathrm{H}_{2}$-IsoP.

Depending on the reductive conditions $\mathrm{H}_{2}$-IsoP may subsequently form several IsoP families. Once the endoperoxide is completely reduced (black and green arrows simultaneously in Scheme 1) it leads to the F-type of IsoPs; a cyclopentane ring with two alcohol functions (in green). If the endoperoxide is partially reduced (arrow in blue and black, one side of the ring only in Scheme 1), the types D- and E- of the IsoPs are produced where the cyclopentane bears a hydroxy-ketone (in blue). The D-type refers to the ketone $(-\mathrm{C}=\mathrm{O})$ moiety on the cyclopentane on the same side as the $\omega$-chain. In the E-type the ketone moiety is on the same side as the $\alpha$-chain. The ratio between F-type, and D- and E-types mainly depends on the number of reductive agents, e.g. glutathione and $\alpha$-tocopherol, in cells [13]. Moreover, under physiological conditions, dehydration can occur especially in the hydroxylketone structures (type D- and E-). Subsequently, A- and J-types (in red Scheme 1) of the IsoPs are formed by the dehydration of E- and D-type respectively. As like D- and E-types, A- and J-types are recognizable with the ketone moieties on the side of the $\alpha$-chain (A-type) or $\omega$-chain (J-type).

Other IsoP metabolites, such as the epoxy-isoprostanes (epoxyIsoPs) are also formed in vivo and detected in mildly oxidized LDL of atherosclerotic lesions $[14,15]$. The epoxy-IsoPs biosynthesis (in orange Scheme 1) follows the same pathway as the formation of the $G_{2}$-IsoP intermediate, but the endoperoxide is first reduced into the hydroperoxy- $\mathrm{D}_{2}$-IsoP compound. Then, dehydration occurs due to the acidity of the $\alpha$-keto hydrogen atom and the hydroperoxy function, leading to the formation of an epoxide (orange arrows Scheme 1) to give epoxy$\mathrm{D}_{2}$-IsoPs. Epoxy- $\mathrm{D}_{2}$-IsoPs were shown to dehydrate spontaneously to 


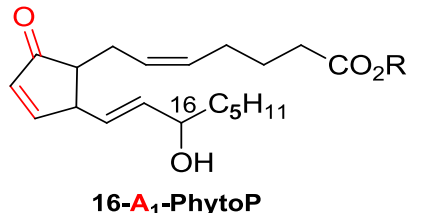

Basic conditions

16-A - -PhytoP<smiles>[R]OC(=O)CCC/C=C/CC1C=CC(=O)C1/C=C/[C@@H]([13CH2])O</smiles>

16-J1-PhytoP<smiles>[R]OC(=O)CCC/C=C/CC1=C(/C=C/[C@H]([10BH2])O)CCC1=O</smiles>

Scheme 2. Generation of tetrasubstituted phytoprostanes (PhytoP) $\mathrm{B}_{1}$ and $\mathrm{L}_{1}$ in basic condition from cyclopentenone derivatives.
epoxy-J $J_{2}$-IsoPs. Recently, epoxy-IsoPs were shown to exert anti-inflammatory activity and may be a valuable group of compounds in inflammatory diseases $[15,16]$.

Two other types of isoprostanoids should also be noted; the B- and L-types (Scheme 2). Under basic conditions, A- and J-types undergo facile isomerization of the double bond. This isomerization is easily explained by the formation of the thermodynamically more stable metabolites. Therefore, the A-type isomerizes into B-type, the J-type leads to the L-type.

However, as AA and other PUFA have several bis-allylic positions in their structures allowing for numerous opportunities for free radical attack, not only the 15-series isoprostanes as shown in Scheme 1 but also 5-, 8- and 12-series isomers can be produced. As described in Scheme 3, hydrogen abstraction on C7 (in red Scheme 3) will give the 5-series of IsoPs, whereas 8- and 12-series are produced through C10 hydrogen abstraction. Consequently, starting from AA, 4 series of IsoPs may be formed, 5-, 8-, 12- and 15-series, and it should be highlighted that the 5- and the 15-series are the most abundant $[17,18]$. Considering the unique formation of F-type IsoPs, the formation of 5 stereogenic centres give rise to 64 different IsoP isomers (enantiomers included).

Both n- 6 and n-3 are essential PUFA in humans. The n-3 PUFA are frequently recommended because of possible health benefits. AA is the main $n-6$ PUFA in human cell membranes, whereas $\alpha$-linolenic acid (ALA, C18:3 n-3) is the main n-3 PUFA in plant leaves. In fact, ALA metabolites, were first discovered by Mueller and co-workers in 1998, and were named phytoprostanes (PhytoPs) [19]. As shown in Scheme 4, ALA contains two bis-allylic positions (C11 and C14) leading to two series of F-type PhytoPs; the 9-series and the 16-series, namely 32 isomers (enantiomers included).

Eicosapentaenoic acid (EPA, C20:5 n-3) together with docosahexaenoic acid (DHA, C22:6 n-3) are mostly provided by the diet from marine resources such as fish or algae. The peroxidation of EPA [20] and its four bis-allylic positions will provide 6 series of IsoPs (5-, 8-, 11-, 12-, 15- and 18-, Scheme 5). The 5- and 18-series are highlighted to be the most abundant series among the other. As for DHA, 8 series can be formed (4-, 7-, 10-, 11-, 13-, 14-, 17- and 20-, Scheme 6). Predominant in the grey matter of the brain, the term neuro- was affixed and the DHA metabolites $[21,22]$ were named NeuroPs. Among the metabolites, the 4- and 20-series were shown to be the most abundant [23].

Mostly found in the white matter of the brain, adrenic acid (AdA, C22:4 n-6) was probably the least considered PUFA, but due to its potential beneficial activities in humans AdA is now more and more studied. In order to differentiate AdA isoprostanoids from the AA derived ones, the term dihomo-isoprostanes (dihomo-IsoPs) was introduced by VanRollins et al. in 2008 [24]. Four series of dihomo-IsoPs are produced by AdA oxidation, namely the 7-, 10-, 14- and 17-series (Scheme 7), with the 7- and 17-series as the most abundant.

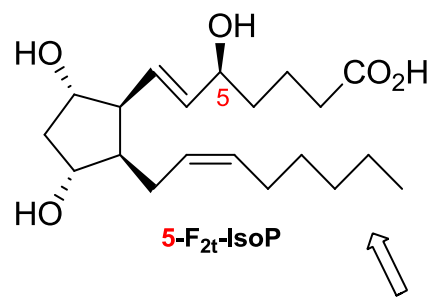<smiles>CCCCC[C@H]1[C@@H](O)C[C@@H](O)[C@@H]1/C=C/[C@@H](O)C/C=C\CCCC(=O)O</smiles>

Scheme 3. The main $\mathrm{F}_{3}$-isoprostane isomers derived from arachidonic acid. IsoP: isoprostane. (For interpretation of the references to color in this figure, the reader is referred to the web version of this article.)<smiles>CCCCC/C=C\C/C=C\CCCC(=O)O</smiles>

Arachidonic acid (AA, C20:4 n-6)

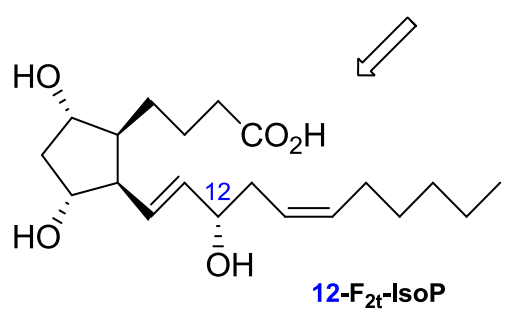

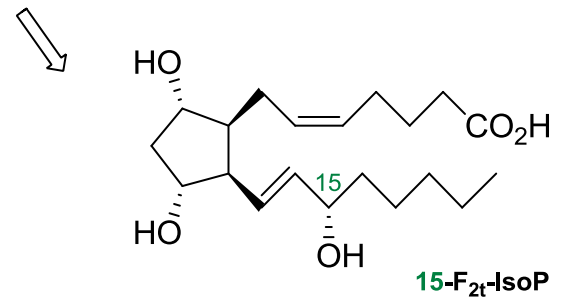




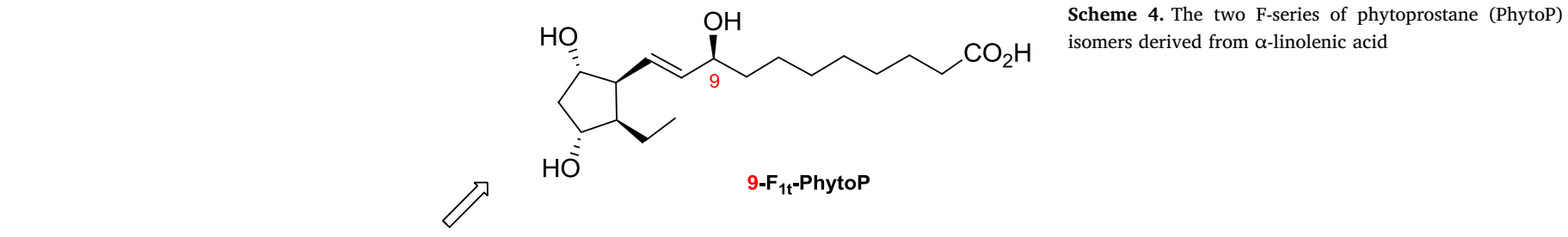

$\mathrm{CO}_{14}=\mathrm{CO}_{2} \mathrm{H}$

$\alpha$-linolenic acid (ALA, C18:3 n-3)
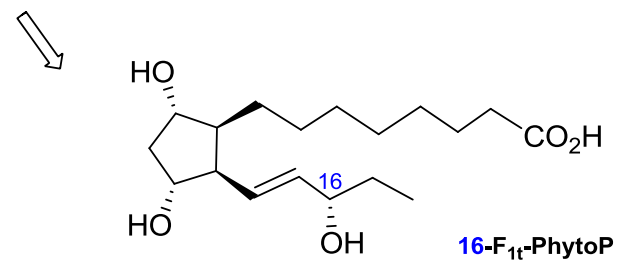

\subsection{The use of organic synthesis to acquire isoprostanoid standards}

The biosynthesis previously described, clearly demonstrates that isoprostanoids are found in biological systems as a complex mixture of isomers. In turn, this raises the question which isomer(s) is of physiological relevance or a possible biomarker? A single solution exists to efficiently answer the questions - by having the pure isomers at hand. Pure synthetic standards are often available from commercial sources, however even if the first IsoPs were discovered almost three decades ago, up to now, less than 10 pure isoprostanoid isomers are commercially available. Pure isoprostanoid sources are needed to better evaluate their role in lipid biology. Through total synthesis, research on isoprostanoids has been significantly advanced in the last decade $[25,26]$.

Throughout the years, the organic chemists' community developed several synthetic strategies that can be assembled together in three main concepts (Scheme 8). To clearly understand this chemistry part of this review, the structure of those isoprostanoids should be perceived. They are composed of one main cyclic core, which can possess different substituents, and to this core are attached two different lateral chains.

The first concept of the synthesis to be applied for these metabolites is to design the full structure of the molecule in a linear fashion and proceed to the formation of the core at the end of the synthesis (route no. 1, Scheme 8). The groups of Taber [27-35] and Durand/Rossi $[36,37]$ used this strategy (Scheme 9). This approach has the advantage to mimic the biosynthesis and the chemist can expect that the late cyclization is accomplishable. However, toward the final intermediate the route is long, often difficult and at the end can be used for a limited variety of compounds.

Then, a second concept was developed. The idea is to realize the cyclization with one of the chains already present in the structure and add the second lateral chain thereafter (route no. 2, Scheme 8). Few groups used this strategy, such as Jahn [38,39] (Scheme 10) and Carreira [40], Weng/Lu [41] for the synthesis of epoxy-IsoPs. The biomimetic side is conserved while convergence and flexibility are brought on.

Finally, today's most preferred strategy is to synthesize the core and introduce the lateral chains one after the other (route nos. 2 and 3, Scheme 8). This strategy was largely applied by Larock/Lee [42], Rokach [43-46], Zanoni [47,48], Durand/Rossi [49-51], Vionnet/Renaud [52], Snapper [53-56] and Galano/Oger [57]. In the three examples shown in Scheme 11, different core structures are prepared before the

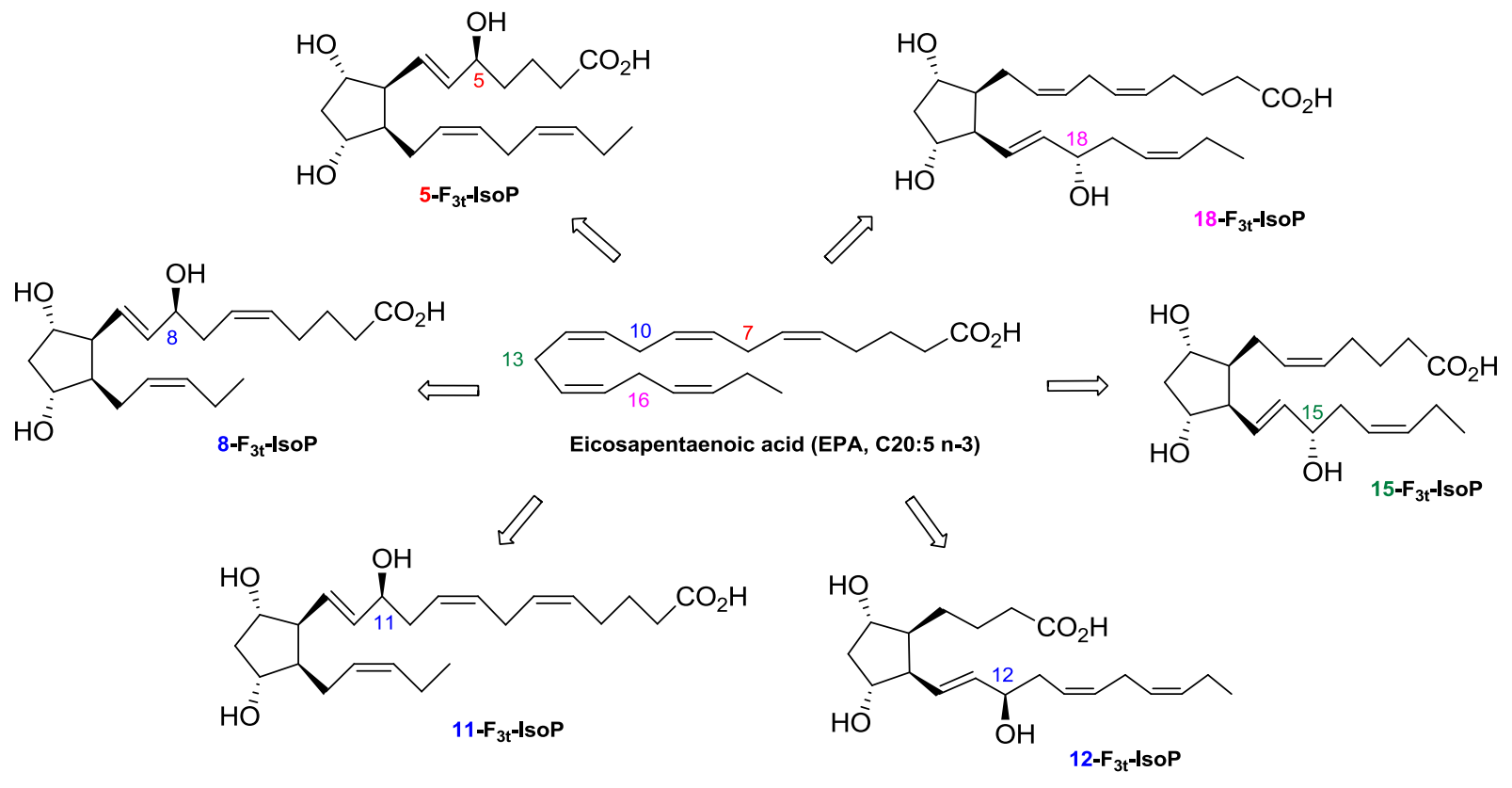

Scheme 5. $\mathrm{F}_{3}$-isoprostane isomers derived from eicosapentaenoic acid. IsoP: isoprostane. 


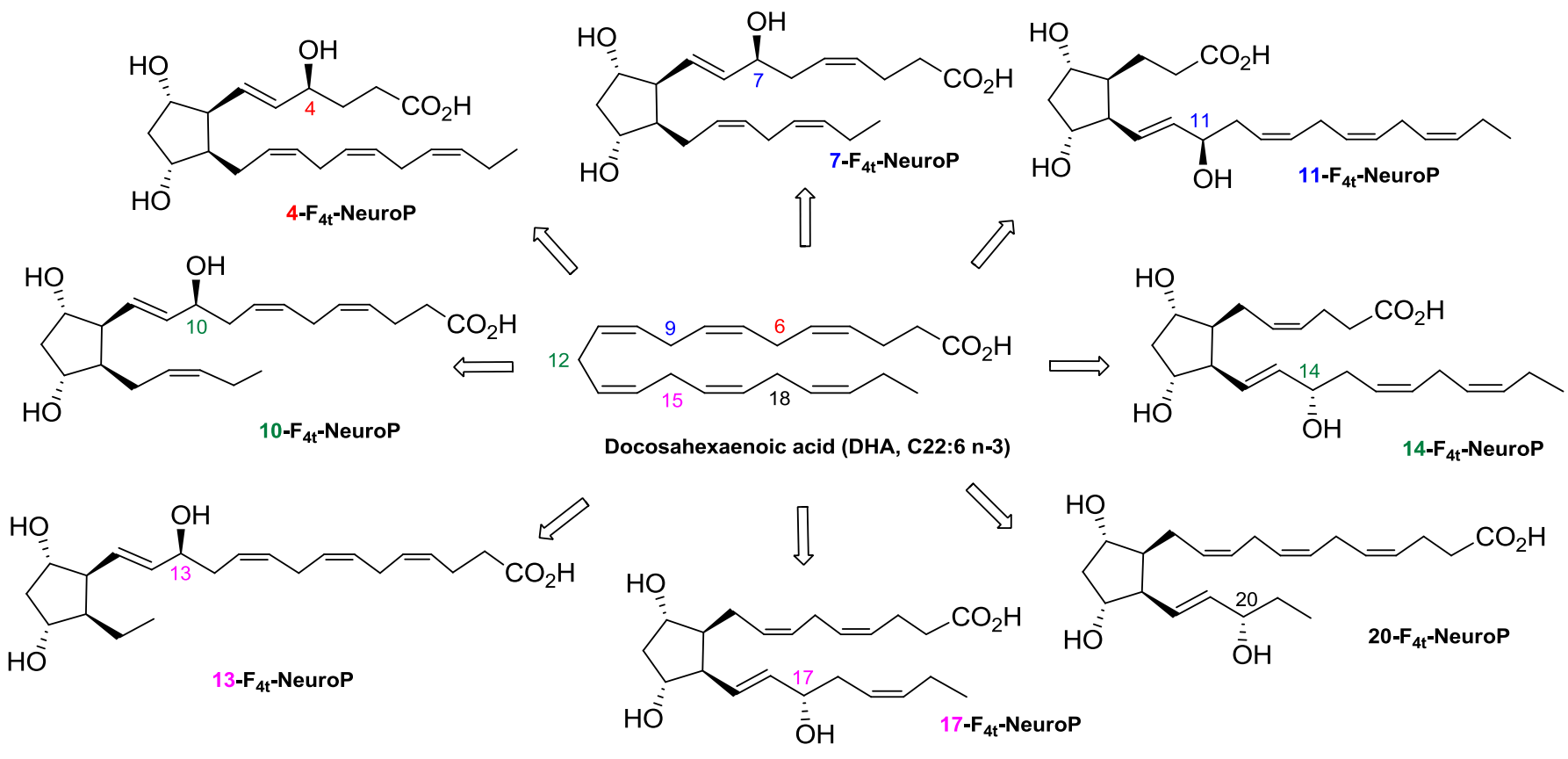

Scheme 6. Neuroprostane (NeuroP) isomers derived from docosahexaenoic acid.

introduction of the first lateral chain, bicyclic (Zanoni, Snapper, Galano/Oger) or with the desired cyclopentane ring and two side groups (the side chains introduced in Galano/Oger Scheme) already in place. This approach is far from the biosynthetic route but has the major advantage to be flexible and will give access to a wide range of isoprostanoids i.e. different types (A, D, E, F and J), different stereochemistry's (cis or trans IsoPs) and allow starting from only one common intermediate. This strategy was also used for the synthesis of epoxyIsoPs by the groups of Acharya/Kobayashi, Jung, Zhong [58-63].

To date, chemists are still developing syntheses of isoprostanoids to gain access to all the families together with their isomers and epimers. The research in the field of isoprostanoids is focused in their use as biomarkers and their biological activities.

\subsection{Metabolism}

While the metabolism of the IsoPs, particularly $15-\mathrm{F}_{2 \mathrm{t}}$-IsoP has been described in detail [64], the metabolism of the NeuroPs and PhytoPs is much less studied. As shown in Fig 1, the main metabolic reactions are $\beta$-oxidation and reduction, respectively. The NeuroPs, $4-\mathrm{F}_{4 \mathrm{t}} \mathrm{-NeuroP}$ and $10-\mathrm{F}_{4 \mathrm{t}}$-NeuroP have been investigated in human and animal studies related to neurological disorders $[23,65]$, and interestingly, $4-\mathrm{F}_{4 \mathrm{t}^{-}}$ NeuroP was found mainly in the circulation and to a lesser extend in the urine whereas $10-\mathrm{F}_{4 \mathrm{t}}$-NeuroP were detectable in both body fluids (Fig 1). The metabolism of PhytoPs is even less well studied where only two reports were found in the literature. Karg's group identified that ingestion of plant oils by healthy males displayed slow absorption and excretion of PhytoPs and likewise, Barden et al. showed that ingestion of ALA (flaxseed oil) augmented $\mathrm{F}_{1}$-PhytoP production in healthy human plasma but not in urine $[8,11]$. Although not yet experimentally proven, it is expected that the metabolism of NeuroPs and PhytoPs proceeds along the same pathways as observed for the IsoPs. As postulated in Fig 1 (dotted lines) $\beta$-oxidation and reduction would lead to 2,3,4,5-tetranor-10- $\mathrm{F}_{4 \mathrm{t}}$-NeuroP and 2,3,4,5,6,7-hexanor-10- $\mathrm{F}_{4 \mathrm{t}}$-NeuroP and $2,3,4,5,6,7$-hexanor- $16-\mathrm{F}_{1}$-PhytoP, three possible urinary metabolites. However, more work will be needed to map the metabolism and excretion routes of the NeuroPs and PhytoPs, helping to establish their use as possible new oxidative stress biomarkers.

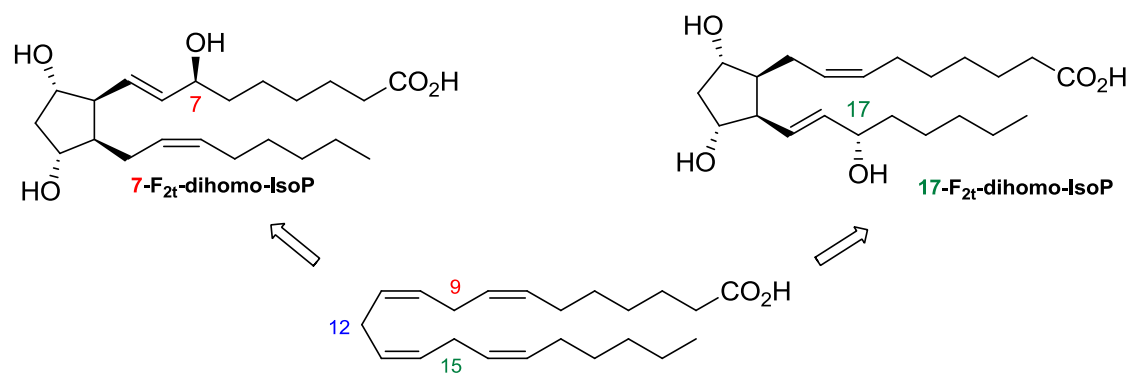

Scheme 7. $\mathrm{F}_{2}$-dihomo-isoprostane (dihomo-IsoP) isomers derived from adrenic acid.

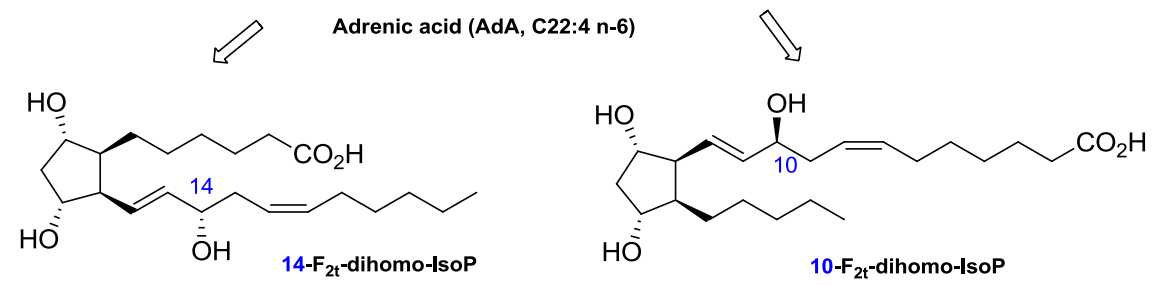



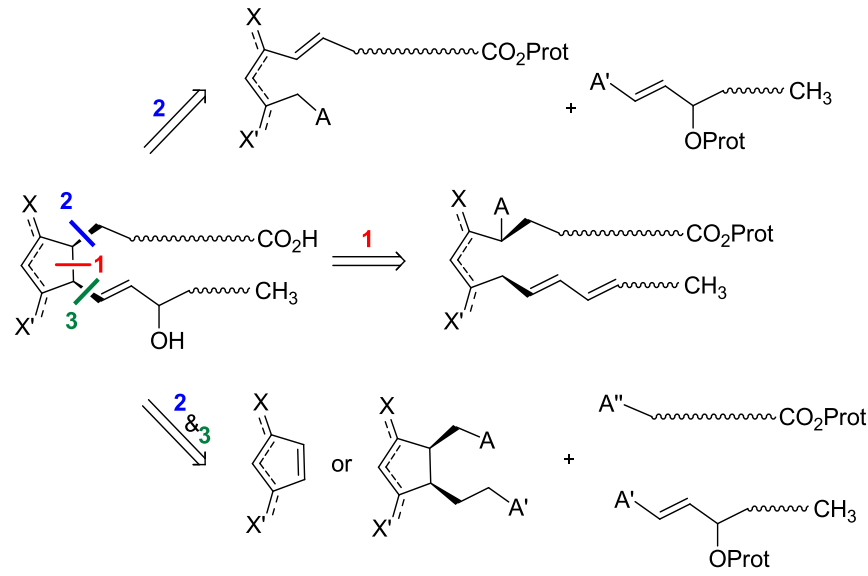

$A, A^{\prime}, A^{\prime \prime}=$ activating groups

$\mathrm{X}=\mathrm{O}, \mathrm{OH}, \mathrm{O}-$ protected

Prot $=$ protection

Scheme 8. Retrosynthetic analysis of the three main synthetic strategies developed for isoprostanoids.

\section{Isoprostanoid analysis}

Morrow's group presented their study about, "one fresh plasma $v s$ several months stored plasma" where they observed an increase in the formation of compounds that also occurred in other biological fluids $[1,66]$. This study marks the discovery of the racemic prostaglandin diastereoisomers which they later termed isoprostanes [67]. In recent years, a wide variety of non-enzymatic metabolites were identified, including AA, AdA, ALA, EPA, docosapentaenoic (DPA) and DHA derived autoxidation products $[68,69]$. From an analytical chemistry point of view, several characteristics make the analysis of isoprostanoids a challenging task. Firstly, to distinguish between enzymatic and non-enzymatic products, isomeric compounds including, diastereomers as well as geometric and positional isomers need to be distinguished [70]. Secondly, the usually low abundance of isoprostanoids demands high analytical sensitivity and thirdly, artefactual formation of isoprostanoids during sample treatment remains an important concern [71]. Several analytical procedures and techniques have arisen over the years; the most important ones will be discussed below.

\subsection{Sample collection}

As mentioned before, due to the high reactivity of the bis-allylic hydrogen atoms, the double bonds found in PUFAs are prone to autoxidation and/or photodegradation, leading to generation of non- enzymatic metabolites including isoprostanoids. In turn, artefactual isoprostanoid formation during bio-sampling, storage, extraction and derivatization is of great concern. It is therefore advised to take several precautions: i) during storage, oxygen should be eliminated from the samples by flushing the samples with nitrogen or argon, ii) a suitable antioxidant such as butylated hydroxytoluene (BHT), triphenylphosphine (TPP), diethylene tri-amine pentaacetic acid (DTPA) and glutathione (GSH) should be used, iii) in order to avoid the ex vivo enzymatic production of eicosanoids, a small amount of a cyclooxygenase inhibitor e.g. indomethacin is commonly added [72] and iv) most importantly all biological fluids and tissues must be kept at ultra-low temperature (i.e. $-80^{\circ} \mathrm{C}$ ) to ensure long term stability (6 months for plasma, several years for urine), ideally avoiding any freeze-thaw cycle. A detailed procedure for sample collection and storage has recently been described by Barden et al. [71].

\subsection{Sample preparation and extraction}

Sample preparation vastly depends on the investigated matrix as well as the subsequently used analysis technique. In general, isoprostanoids can either be extracted from the matrix itself, or after a comprehensive lipid extraction step, following well established protocols such as Folch (chloroform/methanol, 2:1, v/v), Bligh and Dyer (chloroform/methanol, 1:2, v/v) or methyl tert-butyl ether (MTBE) extraction [73-75] are analyzed. For tissues, total (free and bound) isoprostanoids needs to be hydrolysed to release esterified isoprostanoids from the matrix [76]. Hydrolysis is usually performed by adding a methanolic or ethanolic alkaline solution to the sample, followed by gentle heating (e.g. $1 \mathrm{M} \mathrm{KOH}$ in methanol for $30 \mathrm{~min}$ at $37^{\circ} \mathrm{C}$ ) [77]. In the case of urinary samples, isoprostanoids are present in free form thus hydrolysis is not required, nevertheless some authors recommend pretreatment with glucuronidase to cleave glucuronide bound analytes and to reduce matrix effects [78].

The extraction of isoprostanoids from biological matrices usually involves solid phase extraction (SPE). Typical protocols include the use of reversed phase materials [79] and in case of urinary samples, weakanion exchange materials [80]. The latter allows for dedicated washing procedures resulting in clean extracts, even from a highly challenging matrix including urine samples. Another popular extraction procedure, that has particularly been used in combination with gas chromatography mass spectrometry (GC-MS) is thin layer chromatography (TLC) [81]. Other less frequently applied techniques, include: combined SPE and TLC [82] as well as immunoaffinity based techniques, that can be useful for $15-\mathrm{F}_{2 \mathrm{t}}$-IsoP analysis, but not for omega-3 PUFA derived isoprostanoids, due to a lack of specific immobilized antibodies [83].

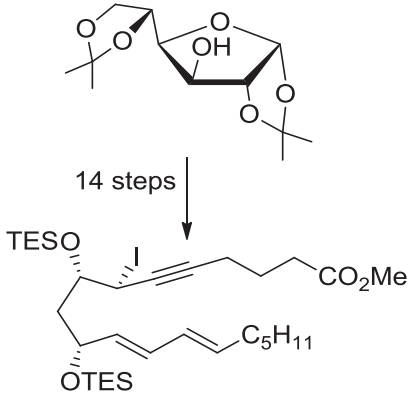

Work of Durand/Rossi and coll.
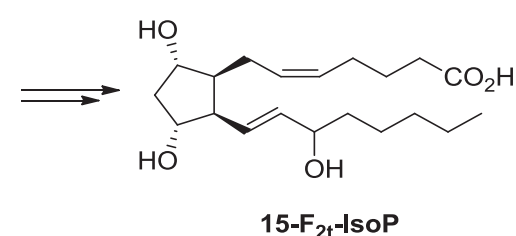

$15-F_{2 t}$-IsoP

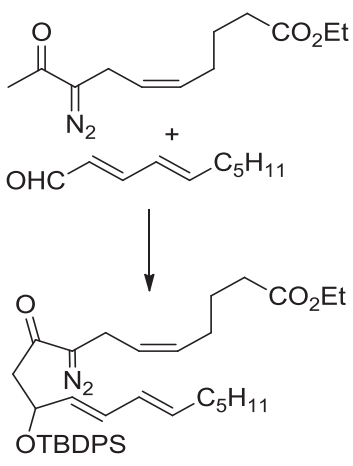

Work of Taber and coll.

Scheme 9. Examples of biomimetic synthetic approaches. 
<smiles>COC(=O)CC(C)=CC=CC=O</smiles><smiles>COC(=O)CC(O)CC([Se-])C=CC=Cc1ccccc1</smiles><smiles>C#C[C@H]1[C@@H](O)[C@@H](/C=C/C(O)CCCCC)[C@H](C/C=C/CCCC(=O)O)[C@H]1O</smiles>

Scheme 10. One chain containing structure prior to cyclization synthetic strategy.

\subsection{Analysis}

In principle, the analysis of isoprostanoids can be divided into immunological assays and mass spectrometry based techniques. However, enzyme linked immunosorbent assays (ELISA kits) and radioimmunoassays (RIA) are limited to a single component, $15-\mathrm{F}_{2 \mathrm{t}}$-IsoP and besides their intrinsic issue regarding specificity, it also presents severe limitations.

$15-\mathrm{F}_{2 \mathrm{t}}$-IsoP

Mass spectrometry based analysis is usually carried out in combination with either gas or liquid chromatography (GC-MS, LC-MS) [81]. Both, GC-MS and LC-MS present specific advantages and disadvantages, which will briefly be discussed below. An excellent in-depth discussion has recently been described by Tsikas and Zoerner [84].

\subsubsection{Derivatization}

For GC-MS analysis, derivatization is mandatory. It is common to

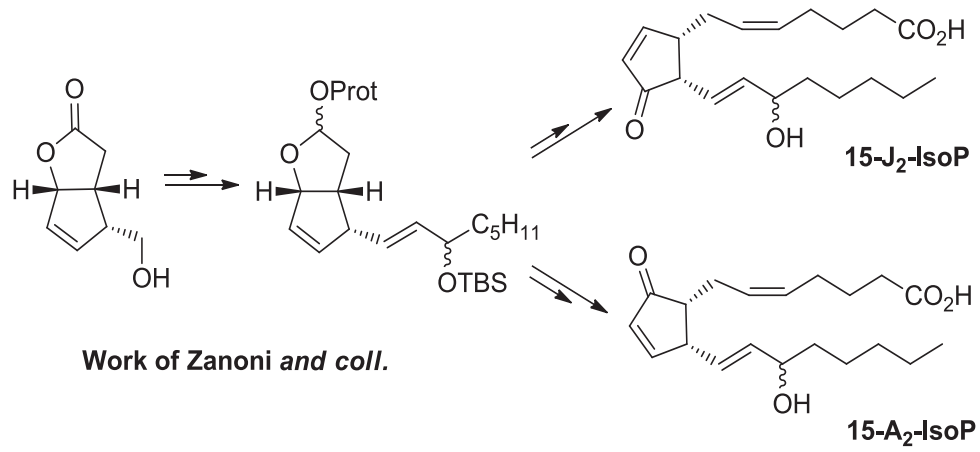

Scheme 11. Examples of approaches for isoprostanoid without pre-introduction of the two chains.

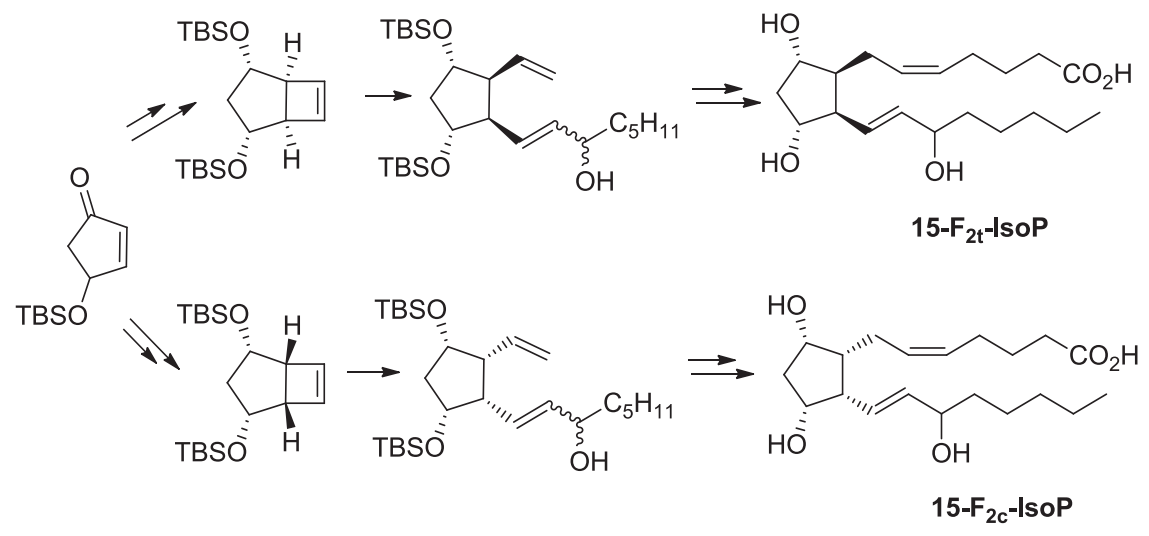

Work of Snapper and coll.

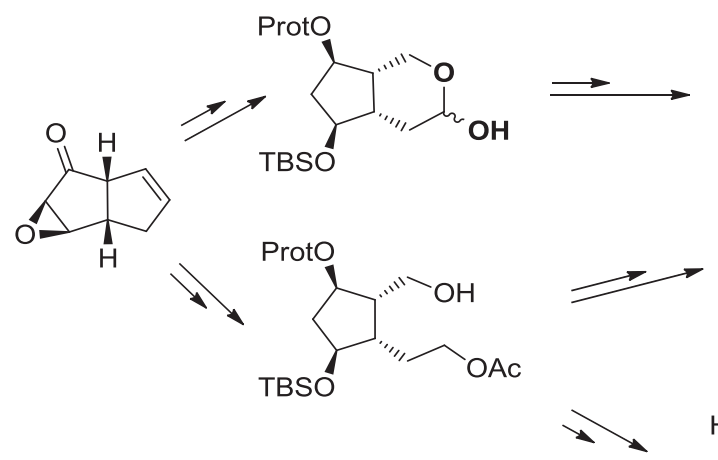

Work of Galano/Oger and coll.<smiles>CCCCCCC(O)/C=C/[C@H]1[C@H](O)C[C@H](O)[C@@H]1C/C=C/CCCC(=O)O</smiles><smiles>CCCCCC(O)/C=C/C1C(=O)C[C@@H](O)[C@@H]1C/C=C/CCCC(=O)O</smiles><smiles>CCC/C=C\C/C=C/C[C@H]1[C@@H](O)C[C@@H](O)[C@@H]1/C=C/[C@H](O)CCC(=O)O</smiles> 


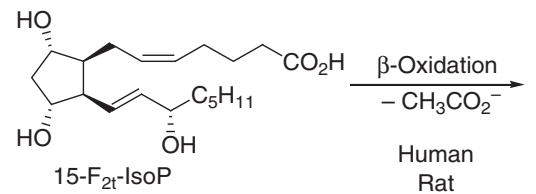

Rat
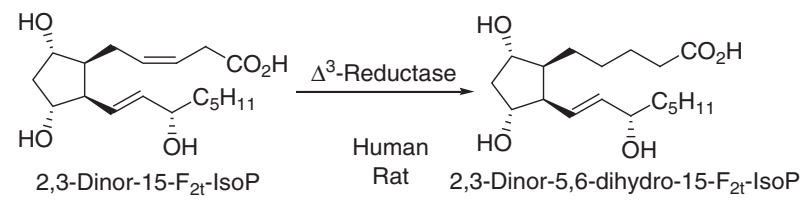
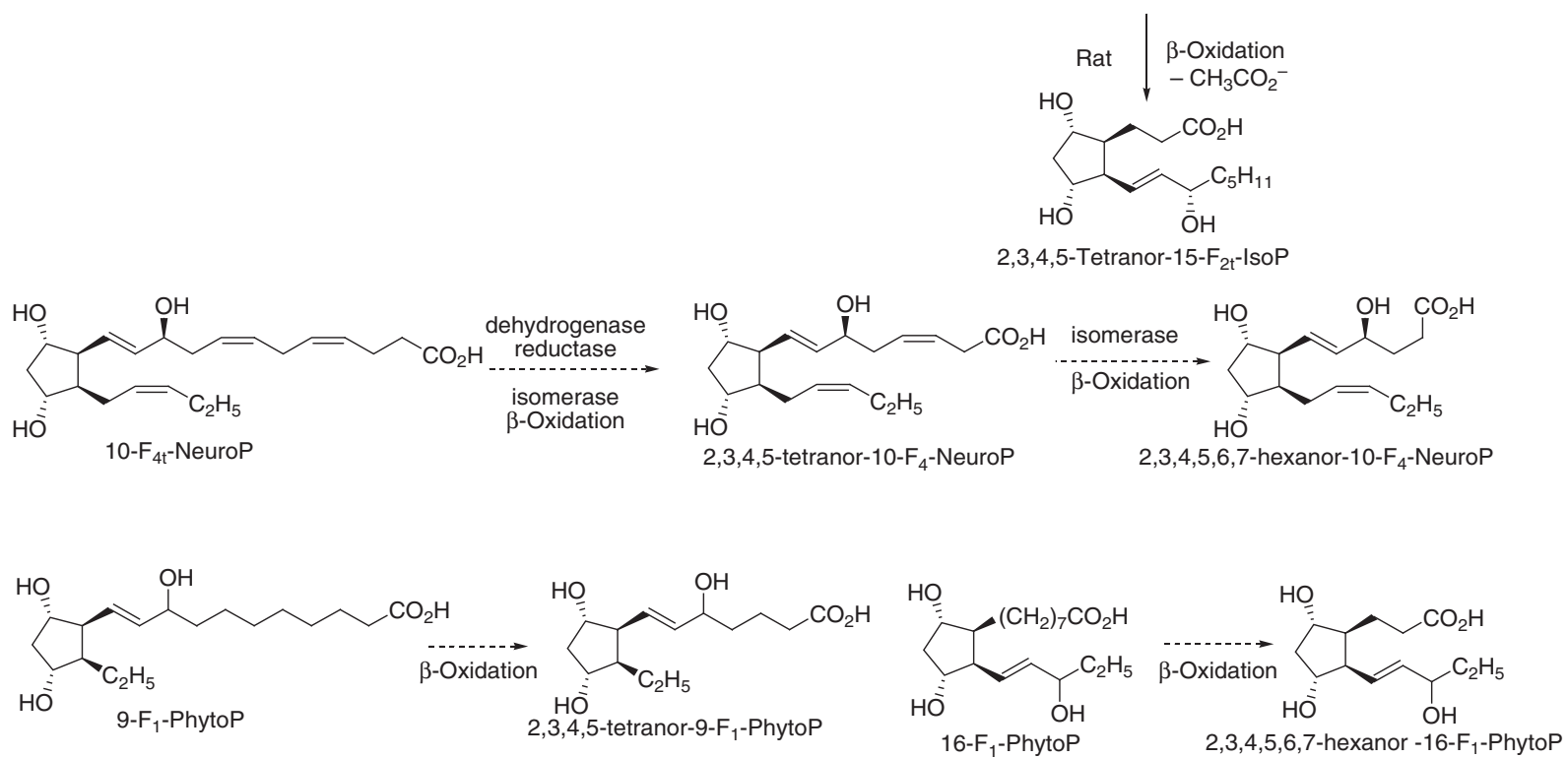

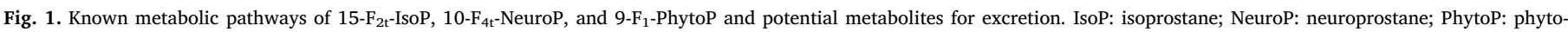
prostane.

initially convert carboxylic acid function into pentafluorobenzyl esters (PFB) by reacting the samples with pentafluorobenzyl bromide (PFBBr) using a base catalyst such as $N, N^{\prime}$-diisopropylethylamine. Subsequently, hydroxyl functions are silylated with either bis-(trimethylsilyl)trifluoroacetamide (BSTFA) or $N$-methyl- $N$-trimethylsilyltrifluoroacetamide with or without the use of a catalyst $[70,85]$. Although derivatization is not mandatory for LC based isoprostanoid analysis, some groups addressed the possibility of facilitating electro-spray ionization in positive mode (ESI + ) using dedicated derivatization strategies as well as using bromine labelled reagents for isotope pattern recognition $[86,87]$. However, to date, no study could clearly prove the benefits of isoprostanoid derivatization prior LC-MS analysis [88]. The reasons for this might be the additional steps needed, kinetic limitation due to the very low analyte concentrations, increased matrix effects caused by residual derivatization reagents as well as over the years increased mass spectrometer sensitivity in negative mode ESI (ESI-), allowing the analysis of native isoprostanoids.

\subsection{2. $G C-M S$ and $G C-M S / M S$ analysis}

Traditionally, isoprostanoids have been analyzed using GC-MS [81]. Even though the GC based analysis requires sample derivatization, the resolving power of the GC is unprecedented. Mainly due to a virtually absent eddy diffusion, GC analysis achieves very high plate numbers and in turn, is ideally suited for the analysis of closely related substances, such as the isoprostanoids. With respect to MS detection, over the years several ionization techniques have been tested, however, electron-capture negative ionization (ECNI) using methane as the reagent gas, has become the gold standard. Especially in combination with PFB esters, ECNI allows the generation of molecular ions, which are usually monitored either in selected-ion monitoring (SIM) or multiple-reaction monitoring (MRM) mode, when using tandem mass spectrometry (GC-MS/MS). Particularly, the latter allows gaining selectivity by choosing analyte specific fragments [89]. Advanced protocols for GC-MS and GC-MS/MS analysis have recently been published by Tsikas et al. [90].

\subsection{3. $L C-M S$ and $L C-M S / M S$ analysis}

Due to the isoprostanoids low abundance and the high selectivity needed for proper isoprostanoid analysis, LC is usually coupled to tandem mass spectrometer (LC-MS/MS), making use of MRM on triplequadrupole or quadrupole linear ion trap (QTrap) type instruments. With respect to the commonly applied ionization techniques, some authors have described the use of atmospheric pressure chemical ionization in the negative mode (APCI-) [91]. However, by far, the most frequently applied technique is ESI-.

LC-MS/MS has the advantage to select analyte characteristic fragment ions, for example related to the position of hydroxyl function within the molecule [92]. A complete set of fragmentation and ion transitions from the precursors of the known isoprostanes are compiled in Table 1 . While carefully chosen MRM transitions allow to readily classify isoprostanes belonging to the $5,8,12$ or 15 -series, the identification of geometric isomers as well as enantiomers, demands chromatographic separation as well as the availability of synthetic standards. An example for the characteristic fragmentation of positional NeuroP isomers are $4-\mathrm{F}_{4 \mathrm{t}}$-NeuroP and $10-\mathrm{F}_{4 \mathrm{t}}$-NeuroP which can be distinguished based on the MRM transitions $m / z$ 377- $>101$ for $4-\mathrm{F}_{4 \mathrm{t}^{-}}$ NeuroP and $m / z$ 377- $>153$ for 10- $\mathrm{F}_{4 \mathrm{t}}$-NeuroP [93] (Table 1).

An upcoming technique which in the future might facilitate the separation of isomers, closely eluting in the LC is ion-mobility-spectrometry [94]. However, to the best of our knowledge applications in the field of isoprostanoid analysis remain very limited [95]. It should be noted that not all the isoprostanoids have been evaluated in biological specimen and analyses of PhytoPs are mainly conducted in food and plant samples whereas those of IsoPs and NeuroPs are mainly assessed in mammalian tissues and fluid. What is lacking is the evaluation of PhytoPs in mammalian tissues since ALA is an essential PUFA required in mammals. Furthermore, it should be highlighted that not all mammalian specimen may contain all the isoprostanoids $[96,97]$ and this may depend on the metabolism and the experimental model evaluated [79].

As reference material, a list of the most abundant MS/MS transitions 
Table 1

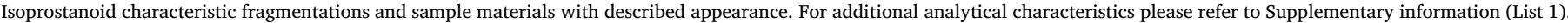

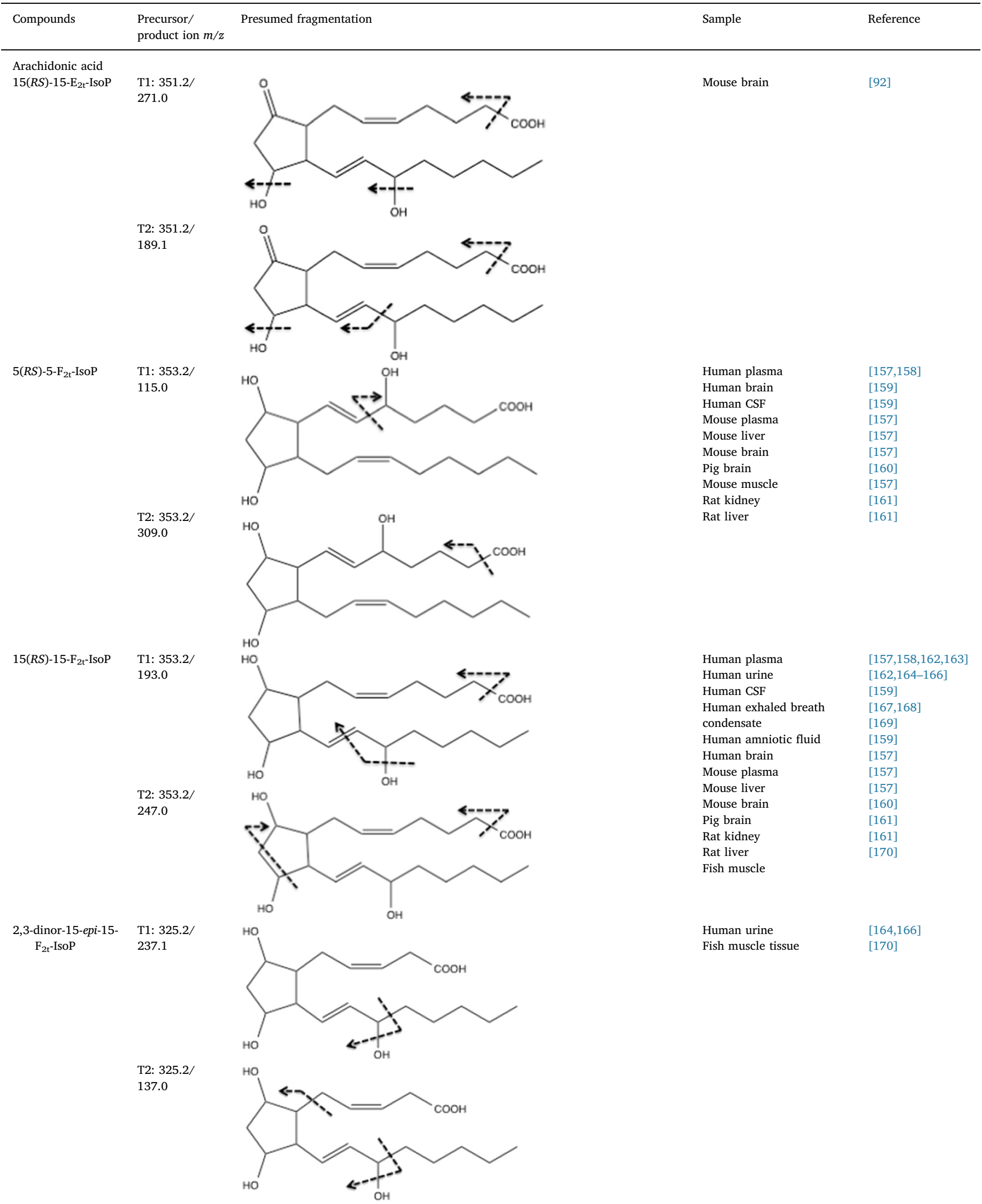


Table 1 (continued)

\begin{tabular}{|c|c|c|c|c|}
\hline Compounds & $\begin{array}{l}\text { Precursor/ } \\
\text { product ion } m / z\end{array}$ & Presumed fragmentation & Sample & Reference \\
\hline \multirow[t]{2}{*}{$\begin{array}{l}\text { ent-2,3-dinor-5,6 } \\
\text { dihydro-15- } \mathrm{F}_{2 \mathrm{t}^{-}} \\
\text {IsoP }\end{array}$} & $\begin{array}{l}\text { T1: } 327.2 / \\
283.2\end{array}$ & но & $\begin{array}{l}\text { Human urine } \\
\text { Fish muscle tissue } \\
\text { Mouse urine }\end{array}$ & $\begin{array}{l}{[164]} \\
{[170]} \\
{[157]}\end{array}$ \\
\hline & $\begin{array}{l}\text { T2: } 327.2 / \\
209.1\end{array}$ & & & \\
\hline \multicolumn{5}{|l|}{ Adrenic acid } \\
\hline \multirow[t]{2}{*}{$\begin{array}{l}\text { 7(RS)-7- } \mathrm{F}_{2 \mathrm{t}} \text {-dihomo- } \\
\text { IsoP }\end{array}$} & $\begin{array}{l}\text { T1: 381.3/ } \\
143.0\end{array}$ & & $\begin{array}{l}\text { Human urine } \\
\text { Pig brain }\end{array}$ & $\begin{array}{l}{[171]} \\
{[160]}\end{array}$ \\
\hline & $\begin{array}{l}\text { T2: } 381.3 / \\
363.1\end{array}$ & & & \\
\hline \multirow[t]{2}{*}{$\begin{array}{l}\text { 17(RS)-17- } \mathrm{F}_{2 \mathrm{t}^{-}} \\
\quad \text { dihomo-IsoP }\end{array}$} & $\begin{array}{l}\text { T1: } 381.3 / \\
319.2\end{array}$ & HO & $\begin{array}{l}\text { Human urine } \\
\text { Pig brain tissue } \\
\text { Mouse brain }\end{array}$ & $\begin{array}{l}{[171]} \\
{[160]} \\
{[157]}\end{array}$ \\
\hline & $\begin{array}{l}\text { T2: } 381.3 / \\
263.1\end{array}$ & & & \\
\hline
\end{tabular}


Table 1 (continued)

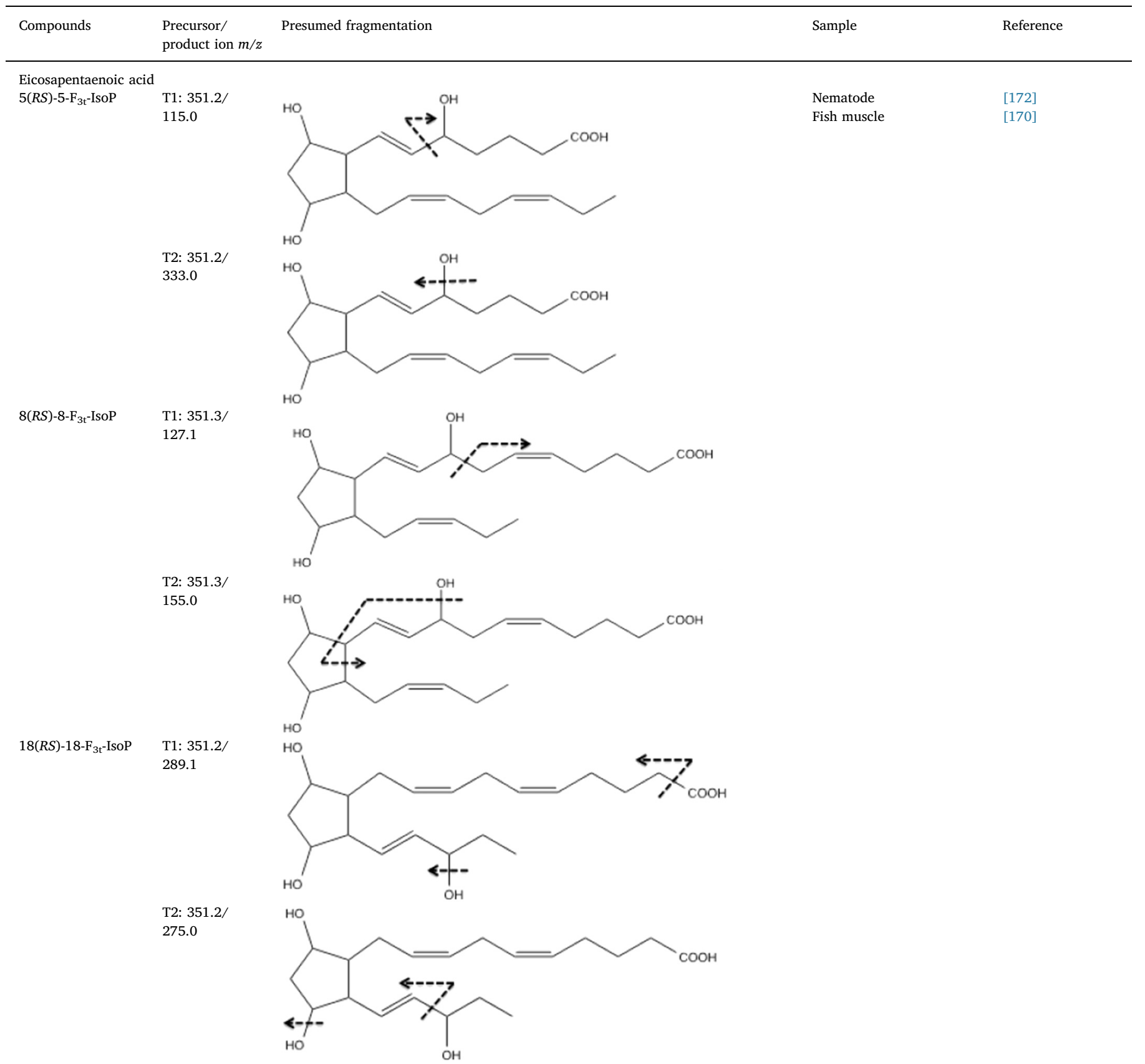

Docosahexaenoic acid

T1: 379.2/

101.0

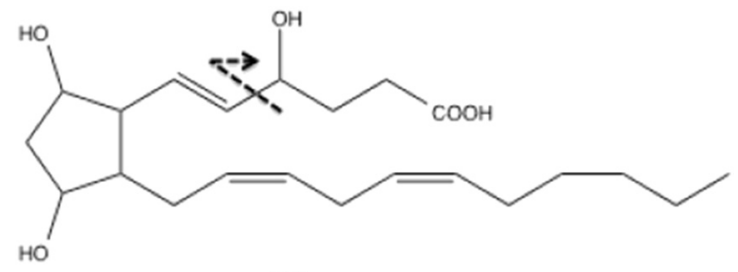

T2: $379.2 /$

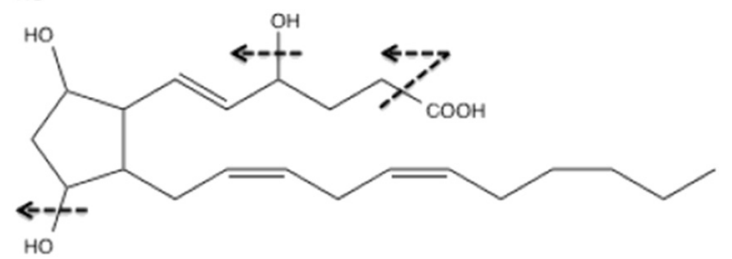

Human urine 
Table 1 (continued)

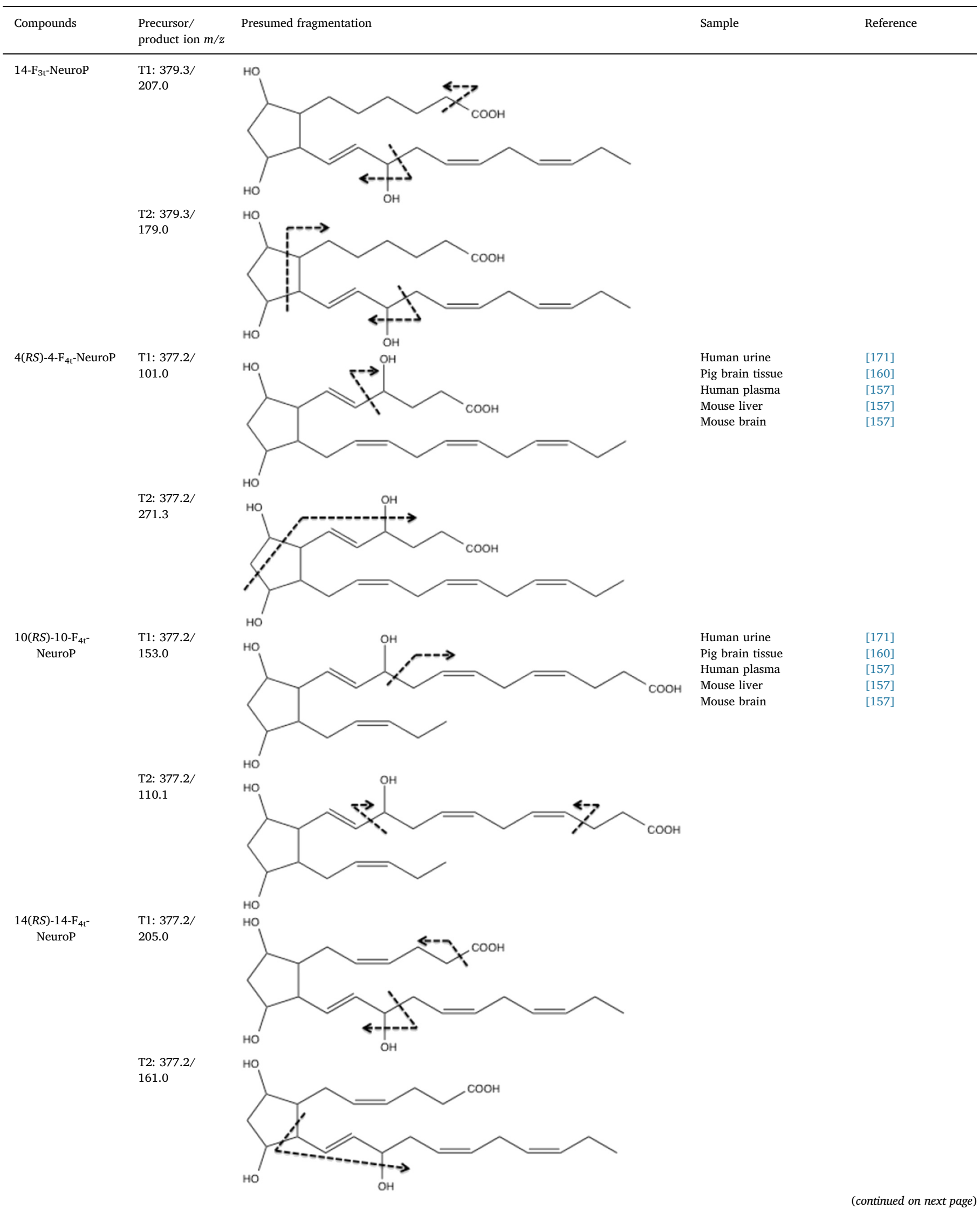


Table 1 (continued)

\begin{tabular}{|c|c|c|c|c|}
\hline Compounds & $\begin{array}{l}\text { Precursor/ } \\
\text { product ion } m / z\end{array}$ & Presumed fragmentation & Sample & Reference \\
\hline \multirow[t]{2}{*}{$\begin{array}{c}20(R S)-20-\mathrm{F}_{4 \mathrm{t}^{-}} \\
\text {NeuroP }\end{array}$} & $\begin{array}{l}\text { T1: } 377.3 / \\
315.2\end{array}$ & & & \\
\hline & $\begin{array}{l}\text { T2: } 377.3 / \\
323.0\end{array}$ & & & \\
\hline
\end{tabular}

$\alpha$-Linolenic acid $16(R S)-16-\mathrm{A}_{1 \mathrm{t}^{-}}$

PhytoP

9(RS)-9-D 1 -PhytoP
T1: 307.2/

289.2

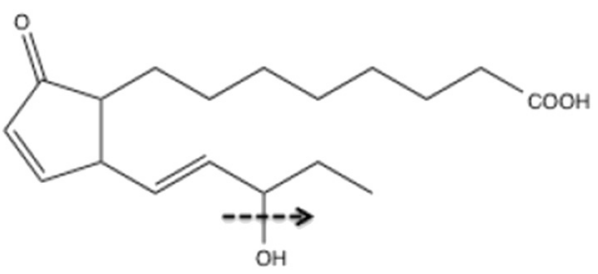

T2: 307.2/

249.0

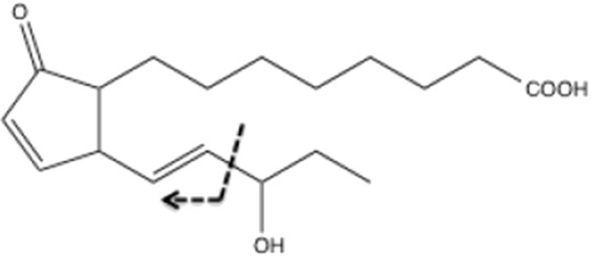

T1: 307.2/

235.0

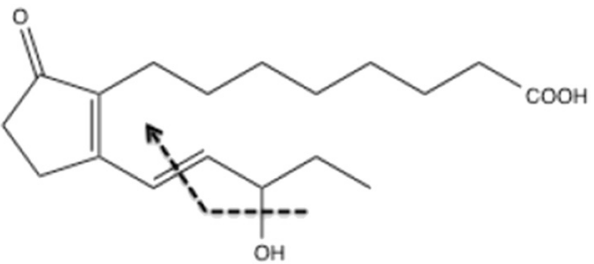

T2: 307.2/

223.0

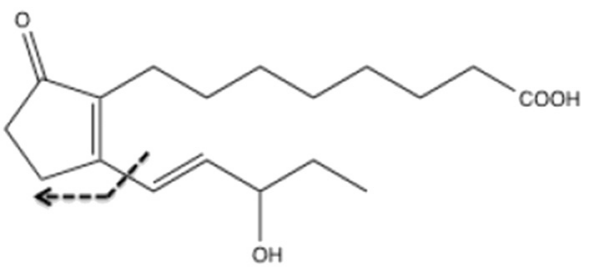

$1: 325.0$

289.0

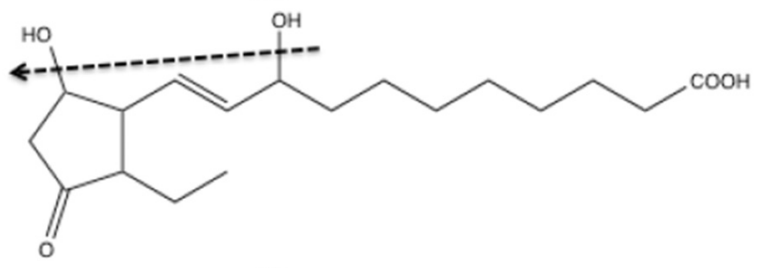

T2: 325.0

123.0

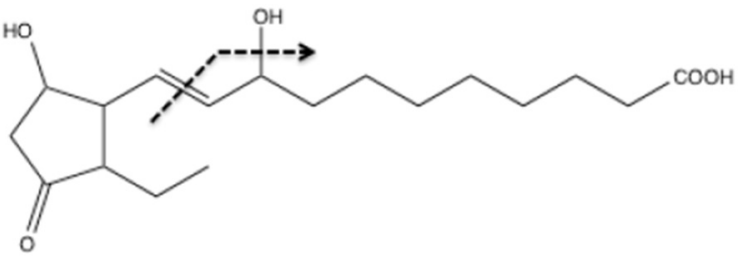

Plant cell culture: $N$.

tabacum, G. $\max , R$.

serpentina, A. tenuis

Walnut, Macadamia, Pecan

Almonds

[173]

oil, Sunflower oil

Passiflora edulis Sims. Shell [175]

Macroalgae

[176]

Melon leaves

[177] $\begin{array}{ll}\text { Walnut, Macadamia, Pecan } & {[173]} \\ \text { Almonds } & {[113]} \\ \text { Olive oil, Sunflower oil } & {[174]} \\ \text { Passiflora edulis Sims. Shell } & {[175]} \\ \text { Macroalgae } & {[176]} \\ \text { Melon leaves } & {[177]}\end{array}$ 
Table 1 (continued)

\begin{tabular}{|c|c|c|c|c|}
\hline Compounds & $\begin{array}{l}\text { Precursor/ } \\
\text { product ion } m / z\end{array}$ & Presumed fragmentation & Sample & Reference \\
\hline \multirow[t]{2}{*}{ 9(RS)-9-F $1 \mathrm{t}^{-}$-PhytoP } & $\begin{array}{l}\text { T1: } 327.2 / \\
283.2\end{array}$ & & $\begin{array}{l}\text { Walnut, Macadamia, Pecan } \\
\text { Almonds } \\
\text { Olive oil, Sunflower oil } \\
\text { Passiflora edulis Sims. Shell } \\
\text { Macroalgae } \\
\text { Melon leaves }\end{array}$ & $\begin{array}{l}{[173]} \\
{[113]} \\
{[174]} \\
{[175]} \\
{[176]} \\
{[177]}\end{array}$ \\
\hline & $\begin{array}{l}\text { T2: } 327.2 / \\
171.2\end{array}$ & & & \\
\hline \multirow[t]{2}{*}{$\begin{array}{l}\text { ent-16(RS)-16- } \mathrm{F}_{1 \mathrm{t}^{-}} \\
\text {PhytoP }\end{array}$} & $\begin{array}{l}\text { T1: } 327.2 / \\
283.2\end{array}$ & $\mathrm{HO}$ & $\begin{array}{l}\text { Walnut, Macadamia, Pecan } \\
\text { Almonds } \\
\text { Olive oil, Sunflower oil } \\
\text { Passiflora edulis Sims. Shell } \\
\text { Macroalgae } \\
\text { Melon leaves }\end{array}$ & $\begin{array}{l}{[173]} \\
{[113]} \\
{[174]} \\
{[175]} \\
{[176]} \\
{[177]}\end{array}$ \\
\hline & $\begin{array}{l}\text { T2: } 327.2 / \\
251.2\end{array}$ & & & \\
\hline \multirow[t]{2}{*}{ 9- $\mathrm{L}_{1 \mathrm{t}}$-PhytoP } & $\begin{array}{l}\mathrm{T} 1: 307.2 / \\
185.1\end{array}$ & & $\begin{array}{l}\text { Walnut, Macadamia, Pecan } \\
\text { Almonds } \\
\text { Olive oil, Sunflower oil } \\
\text { Passiflora edulis Sims. Shell } \\
\text { Macroalgae } \\
\text { Melon leaves }\end{array}$ & $\begin{array}{l}{[173]} \\
{[113]} \\
{[174]} \\
{[175]} \\
{[176]} \\
{[177]}\end{array}$ \\
\hline & $\begin{array}{l}\text { T1: } 307.2 / \\
197.0\end{array}$ & & & \\
\hline
\end{tabular}

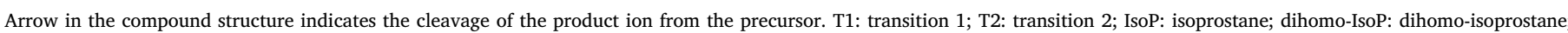
NeuroP: neuroprostane; PhytoP: phytoprostane.

for a series of isoprostanoids is provided in Table 1. In addition, an analytical database containing analytical settings and tandem mass spectra of over 20 isoprostanoids can be found in the Supplementary Information (List 1).

\section{Biomarkers in biological systems}

Since the discovery of IsoPs, $15-\mathrm{F}_{2 \mathrm{t}}$-IsoP has been labelled as the biomarker for oxidative stress in human diseases. Notably, isoprostanoid measurements have been performed in mammalian plasma, urine, cerebrospinal fluid, sputum, saliva, exhaled breath condensate, brain tissues, atherosclerotic plaques and even gastric mucosa. More recently, the use of IsoPs as biomarkers has been extended to the marine ecosystem and includes environmental studies, and should be recognized that one large part of PUFA in the human diet is obtained through our marine resources, e.g. seafood and algae. Notwithstanding, plant oil is also an important source of PUFA for the human body. Studies on the use of PhytoPs from ALA as biomarkers to evaluate plant oil oxidation from seeds, nuts, leaves, olives and wine have been reported recently. The quality of both marine and plant oils is important for general health and well-being as they are the source of essential fatty acids in our diet; without doubt, increase in oxidative stress biomarkers are good indicators for the PUFA quality. Although NeuroPs were utilized to measure oxidative stress related to diseases, this has not been fully utilized for measurement in the marine ecosystem. Yet, the extent of use as biomarkers in human studies is not large for NeuroPs compared to IsoPs, but has shown to be an important indicator in studies of oxidative stress related to neurodegeneration and neurological 
conditions. In this part, we will explore reports on the use of isoprostanoid biomarkers and their applications in different biological areas. Not all studies on human diseases and IsoP biomarkers are included, as to date (2017), > 2500 articles related to isoprostanes are found in PubMed. Only those reports on prospective studies will be highlighted.

\subsection{Isoprostanes}

\subsubsection{Human diseases}

Lipid peroxidation is a critical component of oxidative stress, which is a common biological condition in a wide range of diseases such as, but not limited to, neurological disorders, cardiovascular diseases, diabetes and renal dysfunction [69]. Since its discovery, levels of $\mathrm{F}_{2}$ IsoPs, especially $5-\mathrm{F}_{2 \mathrm{t}}$-IsoP and $15-\mathrm{F}_{2 \mathrm{t}}$-isoP, and their metabolites are routinely measured in non-invasive patient samples, predominantly in blood plasma and urine. Arachidonic acid is esterified in membranes and it is a ubiquitous component of mammalian cell membranes. Elevation of $\mathrm{F}_{2}$-IsoPs in vivo represents the presence of cellular oxidant injury. The detection and quantification of $\mathrm{F}_{2}$-IsoPs and its metabolites has been described in Section 3.

So far, ECNI-GC-MS remains the gold standard for $\mathrm{F}_{2}$-IsoPs measurement due to its superior selectivity and sensitivity. Table 2 reviews the use of 5- and 15-series $\mathrm{F}_{2}$-IsoPs and $15-\mathrm{F}_{2 \mathrm{t}}$-IsoP metabolites as biomarkers in several human diseases relating to oxidative stress. Although in vitro studies and in animal models using $\mathrm{F}_{2}$-IsoPs has remarkably shown to be a reliable oxidant injury biomarker, the same does not hold true for all oxidative stress related diseases in humans that was illustrated by van't Erve et al. [98]. After meta-analysis of studies in different disease conditions, the authors pointed out that 'total' $15-\mathrm{F}_{2 \mathrm{t}}$-IsoP should be considered before interpreting non-enzymatic oxidative damage as generation of 'free' $15-\mathrm{F}_{2 \mathrm{t}}$-IsoP can be induced by inflammation-induced prostaglandin endoperoxide synthase [98]; further studies are needed to justify this finding. Similarly, it is worth mentioning that the experimental results in Table 2 indicate, regardless of the samples used, conflicting data is noted and failed to prove that either $\mathrm{F}_{2}$-IsoPs or $15-\mathrm{F}_{2 \mathrm{t}}$-IsoP metabolites are suitable biomarkers for widespread diseases, such as Alzheimer's disease and type 2 diabetes. While few reports on less prevalent diseases like dengue fever [99] and periodontal disease [100] were distinguished, $\mathrm{F}_{2}$-IsoPs appear to be significant biomarkers of these diseases. Thus, $\mathrm{F}_{2}$-IsoPs or their metabolites might not be suitable biomarkers for all human diseases related to oxidative stress. Nonetheless, it is worth noting that the elevation of $15-\mathrm{F}_{2 \mathrm{t}}$-IsoP in cardiovascular diseases is more consistent than those in neurological disorders, and it is not surprising as $15-\mathrm{F}_{2 \mathrm{t}^{-}}$ IsoP is a vasoconstrictor. So far, although $\mathrm{F}_{2}$-IsoPs are widely recognised as the biomarker for oxidative stress, no large-scale population study has been conducted using the MS platforms and, therefore, warrants a need to validate if it is a good biomarker for all oxidative stress related diseases, including neurodegenerative diseases.

In contrast to $\mathrm{F}_{2}$-IsoPs, AdA-derived $\mathrm{F}_{2}$-dihomo-IsoPs have shown to be potential biomarkers for brain white matter related conditions, where AdA is highly abundant. Studies in patients with neurological disorders, such as Rett Syndrome [101] showed significant elevation of plasma $\mathrm{F}_{2}$-dihomo-IsoPs, and urinary $7-\mathrm{F}_{2 \mathrm{t}}$-dihomo-IsoPs. $17-\mathrm{F}_{2 \mathrm{t}}$-dihomo-IsoPs were shown to be higher in epileptic patients compared to controls [102]. In a recent report, $\mathrm{F}_{2}$-dihomo-IsoPs were used as biomarker of the neuromotor system in adult triathletes. The study showed that physical exercise exerted different responses in the type of $\mathrm{F}_{2}$-dihomo-IsoPs; urinary $17-\mathrm{F}_{2 \mathrm{t}}$-dihomo-IsoPs were elevated and $7-\mathrm{F}_{2 \mathrm{t}}$-dihomo-IsoPs decreased with increment of training load [103].

\subsubsection{Marine ecosystem and environmental stressors}

The use of $\mathrm{F}_{2}$-IsoPs as biomarker for in vivo oxidant injury is not only limited to human diseases, but were also found in marine fishes as well as humans and animals that were exposed to environmental stressors
Table 2

$15-\mathrm{F}_{2 \mathrm{t}}$-IsoPs and metabolites $\left(15-\mathrm{F}_{2 \mathrm{t}}\right.$-IsoP-M) and $5-\mathrm{F}_{2 \mathrm{t}}$-IsoPs $(+)$ used as biomarkers to indicate human disease status related to oxidative stress published up to 2016 .

\begin{tabular}{llll}
\hline Subjects & Sample & Disease & Method Reference \\
& vs \\
& control & \\
& & \\
& &
\end{tabular}

Neurological

Alzheimer's disease

Brain tissue (19)
Urine (9)
Plasma
Free (12)
Total (12
CSF (41)
Urine (56)
$15-F_{2 t^{-}}$IsoP
$15-F_{2 t^{-}}$IsoP-M
Urine $^{+}(50)$
Plasma
CSF $^{+}(50)$
Brain tissue (5)
Plasma (49)
CSF (9)
Brain tissue (6)
Brain tissue (7)
Plasma (47)
Plasma (25)
Anterior cingulate
cortex (9)
Occipital cortex
(9)

Schizophrenic

Huntington's disease

Plasma (134)

Urine (11)

Plasma

Free (20)

Total (19)

Mild cognitive impairment

Urine $^{+}(33)$

Plasma $^{+}$(33)

$\mathrm{CSF}^{+}$(33)

Plasma (47)

CSF (9)

Multiple system atrophy

Dementia

Stroke

Brain tissue (4)

$\uparrow$

GC-MS

[178]

$\leftrightarrow \quad$ GC-MS

[179]

$\leftrightarrow$

GC-MS

$\leftrightarrow$

GC-MS

C-MS

[181]

$\leftrightarrow$

GC-MS

GC-MS

GC-MS

GC-MS

GC-MS

GC-MS

GC-MS

GC-MS

Plasma (21)

Total

Free

Urine

Plasma (44)

Urine (82)

Brain injury CSF (18)

GC-MS

$\leftrightarrow$

GC-MS

GC-MS

[184]

$\leftrightarrow$

GC-MS

[182]

$\leftrightarrow$

GC-MS

hemorrhage (aSAH)

Traumatic brain injury (TBI)

\section{Epilepsy}

Down syndrome

Reversible cerebral vasoconstriction syndrome

Autism (children)

Intermittent explosive disorder

Hypoxic-ischemic encephalopathy

Neuroborreliosis
Plasma (29)

Plasma (44)

CSF (18)

Plasma (15)

CSF (15)

Urine (60)

Plasma (34)

Urine (48)

$15-F_{2 t}-$ IsoP-M

Urine (103)

Plasma (69)

Plasma (22)

Total

Free

Urine (22)

CSF (22)
Cord serum (20)
GC-MS

[191]

ELISA

LC-MS/MS [194]

LC-MS/MS

GC-MS

ELISA

ELISA

[196]

[197]

[198]

LC-MS/MS [199]

LC-MS/MS

[200] 
Table 2 (continued)

\begin{tabular}{|c|c|c|c|c|}
\hline Subjects & Sample & $\begin{array}{l}\text { Disease } \\
\text { vs } \\
\text { control }\end{array}$ & Method & Reference \\
\hline \multicolumn{5}{|l|}{ Cardiovascular } \\
\hline $\begin{array}{l}\text { Asymptomatic and } \\
\text { symptomatic } \\
\text { atherosclerosis }\end{array}$ & $\begin{array}{l}\text { Carotid plaques } \\
\text { (30) }\end{array}$ & $\uparrow$ & LC-MS/MS & [201] \\
\hline $\begin{array}{l}\text { Mild-Moderate } \\
\text { hypertension }\end{array}$ & Urine (30) & $\leftrightarrow$ & GC-MS & {$[202]$} \\
\hline \multirow[t]{6}{*}{ Hypertension } & Plasma (155) & $\leftrightarrow$ & GC-MS & [203] \\
\hline & Urine (155) & $\leftrightarrow$ & & \\
\hline & Urine (31) & $\uparrow$ & ELISA & [204] \\
\hline & Plasma (22) & $\uparrow$ & ELISA & [205] \\
\hline & Urine (22) & $\uparrow$ & & \\
\hline & Urine (116) & $\leftrightarrow$ & ELISA & {$[206]$} \\
\hline \multirow{2}{*}{$\begin{array}{l}\text { Obstructive sleep } \\
\text { apnea }\end{array}$} & Plasma (128) & $\uparrow$ & ELISA & [207] \\
\hline & Urine (86) & $\uparrow$ & LC-MS/MS & [208] \\
\hline Coronary artery disease & Urine (799) & $\uparrow$ & ELISA & [209] \\
\hline $\begin{array}{l}\text { Peripheral artery } \\
\quad \text { disease }\end{array}$ & Plasma (115) & $\uparrow$ & ELISA & [210] \\
\hline Heart failure & Plasma (36) & $\leftrightarrow$ & GC-MS & {$[211]$} \\
\hline \multicolumn{5}{|l|}{ Cancer } \\
\hline Prostate & Urine (304) & $\uparrow$ & ELISA & [212] \\
\hline $\begin{array}{l}\text { Uterine leiomyomas } \\
\text { (fibroids) }\end{array}$ & Urine (20) & $\leftrightarrow$ & ELISA & [213] \\
\hline Breast & Urine (79) & $\leftrightarrow$ & RIA & [214] \\
\hline $\begin{array}{l}\text { Esophageal } \\
\text { adenocarcinoma }\end{array}$ & $\begin{array}{l}\text { Proximal gastric } \\
\text { mucosa (24) }\end{array}$ & $\uparrow$ & ELISA & [215] \\
\hline \multicolumn{5}{|l|}{ Pulmonary dysfunction } \\
\hline Asthma & Sputum (71) & $\uparrow$ & ELISA & [216] \\
\hline \multicolumn{5}{|l|}{ Diabetes } \\
\hline \multirow[t]{13}{*}{ Type 2 DM } & Plasma (10) & $\leftrightarrow$ & ELISA & [217] \\
\hline & Urine (10) & $\uparrow$ & & \\
\hline & Plasma (20) & $\uparrow$ & ELISA & [216] \\
\hline & Plasma (21) & $\uparrow$ & LC-MS & {$[218]$} \\
\hline & Urine (21) & $\uparrow$ & & \\
\hline & Plasma (8) & $\leftrightarrow$ & GC-MS & [219] \\
\hline & Plasma (40) & $\uparrow$ & GC-MS & {$[220]$} \\
\hline & Free & $\uparrow$ & & \\
\hline & Esterified & & & \\
\hline & Urine & $\uparrow$ & & \\
\hline & $15-F_{2 t}-I s o P$ & $\uparrow$ & & \\
\hline & $15-F_{2 t}-I s o P-M$ & & & \\
\hline & $\begin{array}{l}\text { Atherosclerotic } \\
\text { plaques (5) }\end{array}$ & $\downarrow$ & GC-MS & {$[221]$} \\
\hline \multirow[t]{2}{*}{ Type 1 DM } & Plasma (38) & $\leftrightarrow$ & RIA & {$[222]$} \\
\hline & Urine (38) & $\leftrightarrow$ & & \\
\hline \multirow[t]{7}{*}{ Metabolic syndrome } & Plasma (10) & $\uparrow$ & ELISA & {$[223]$} \\
\hline & Plasma (45) & $\leftrightarrow$ & GC-MS & {$[224]$} \\
\hline & Free & $\leftrightarrow$ & & \\
\hline & Esterified & & & \\
\hline & Urine & $\leftrightarrow$ & & \\
\hline & $15-F_{2 t}-I s o P$ & $\leftrightarrow$ & & \\
\hline & $15-F_{2 t}-I s o P-M$ & & & \\
\hline \multicolumn{5}{|l|}{ Renal dysfunction } \\
\hline \multirow[t]{4}{*}{ Nephrotic syndrome } & Plasma (14) & $\leftrightarrow$ & GC-MS & [225] \\
\hline & Urine (14) & & & \\
\hline & $15-F_{2 t^{-}}-I s o P$ & $\leftrightarrow$ & & \\
\hline & $\begin{array}{l}\text { 2,3-dinor-8-iso- } \\
\text { PGF2a }\end{array}$ & $\leftrightarrow$ & & \\
\hline \multirow[t]{3}{*}{ Chronic kidney disease } & Plasma (60) & $\uparrow$ & GC-MS & {$[226]$} \\
\hline & Plasma (184) & $\uparrow$ & GC-MS & {$[227]$} \\
\hline & Plasma (87) & $\leftrightarrow$ & GC-MS & {$[228]$} \\
\hline $\begin{array}{c}\text { Acute renal graft } \\
\text { dysfunction }\end{array}$ & Serum (55) & $\uparrow$ & ELISA & [229] \\
\hline \multicolumn{5}{|l|}{ Liver } \\
\hline \multirow[t]{2}{*}{ Biliary cirrhosis } & Plasma (41) & $\uparrow$ & ELISA & [230] \\
\hline & Urine (41) & $\uparrow$ & & \\
\hline \multirow[t]{2}{*}{ Autoimmune hepatitis } & Plasma (33) & $\uparrow$ & ELISA & {$[231]$} \\
\hline & Urine (33) & $\uparrow$ & & \\
\hline \multirow[t]{3}{*}{ Alcoholic liver disease } & Plasma (24) & $\uparrow$ & ELISA & {$[232]$} \\
\hline & Urine (24) & $\uparrow$ & & \\
\hline & Plasma (63) & $\uparrow$ & ELISA & {$[233]$} \\
\hline
\end{tabular}

Table 2 (continued)

\begin{tabular}{|c|c|c|c|c|}
\hline Subjects & Sample & $\begin{array}{l}\text { Disease } \\
\text { vs } \\
\text { control }\end{array}$ & Method & Reference \\
\hline $\begin{array}{l}\text { Non-alcoholic fatty } \\
\text { liver disease }\end{array}$ & Plasma (90) & $\uparrow$ & ELISA & [233] \\
\hline \multicolumn{5}{|l|}{ Others } \\
\hline Thalassemia & $\begin{array}{l}\text { Plasma (17) } \\
\text { Urine (17) }\end{array}$ & $\begin{array}{l}\uparrow \\
\uparrow\end{array}$ & GC-MS & [234] \\
\hline Dengue fever & $\begin{array}{l}\text { Plasma (35) } \\
\text { Total } \\
\text { Free } \\
\text { Urine (19) }\end{array}$ & $\begin{array}{l}\uparrow \\
\uparrow \\
\uparrow\end{array}$ & GC-MS & [99] \\
\hline \multirow[t]{3}{*}{ Preeclampsia } & $\begin{array}{l}\text { Amniotic fluid } \\
(46)\end{array}$ & $\uparrow$ & LC-MS & [235] \\
\hline & $\begin{array}{l}\text { Plasma-Maternal } \\
\text { (23) } \\
\text { Plasma-Cord (23) }\end{array}$ & $\begin{array}{l}\leftrightarrow \\
\leftrightarrow\end{array}$ & GC-MS & [236] \\
\hline & $\begin{array}{l}\text { Plasma (33) } \\
\text { Plasma }^{+}(33)\end{array}$ & $\begin{array}{l}\leftrightarrow \\
\uparrow\end{array}$ & LC-MS/MS & [237] \\
\hline $\begin{array}{l}\text { Sepsis related to renal, } \\
\text { hepatic, and } \\
\text { coagulation failure }\end{array}$ & Plasma (15-19) & $\uparrow$ & GC-MS & [238] \\
\hline Periodontal disease & Saliva (50) & $\uparrow$ & LC-MS/MS & [100] \\
\hline Influenza A & $\begin{array}{l}\text { Plasma (35) } \\
\text { Urine (35) }\end{array}$ & $\begin{array}{l}\uparrow \\
\leftrightarrow\end{array}$ & GC-MS & [239] \\
\hline Oral lichen planus & Plasma (31) & $\uparrow$ & ELISA & [240] \\
\hline Critically ill patients & Plasma (38) & $\uparrow$ & LC-MS/MS & [241] \\
\hline $\begin{array}{l}\text { Community acquired } \\
\text { pneumonia }\end{array}$ & Serum (50) & $\uparrow$ & ELISA & [242] \\
\hline \multirow[t]{2}{*}{ Rheumatoid arthritis } & Plasma (73) & & LC-MS/MS & [243] \\
\hline & $\begin{array}{l}\text { Free } \\
\text { Esterified } \\
\text { Urine (73) }\end{array}$ & $\begin{array}{l}\downarrow \\
\uparrow \\
\uparrow\end{array}$ & & \\
\hline Paediatric sepsis & Plasma (42) & $\uparrow$ & ELISA & [244] \\
\hline $\begin{array}{l}\text { Helicobacter pylori } \\
\text { infection }\end{array}$ & $\begin{array}{l}\text { Exhaled breath } \\
\text { condensate (41) }\end{array}$ & $\uparrow$ & ELISA & [245] \\
\hline
\end{tabular}

Annotations indicate $\uparrow$ : increase, $\downarrow$ decrease and $\leftrightarrow$ no change compared to controls Numbers in the parentheses are the number of subjects in the study. ELISA: enzymelinked immunosorbent assay; GC: gas chromatography; LC: liquid chromatography; MS: mass spectrometry.

such as the ones listed in Table 3. Most of these studies were reported within the last decade, showing that the detection of $\mathrm{F}_{2}$-IsoPs is starting to receive significant attention in the environmental and marine science areas. Although not all treated marine fishes showed changes in the level of $\mathrm{F}_{2}$-IsoPs in their samples, deep-frozen catfish, $\mathrm{Fe}^{3+}$-treated Fathead minnow fish, and $\mathrm{H}_{2} \mathrm{O}_{2}$-treated medaka fish consistently exhibited an elevation of $\mathrm{F}_{2}$-IsoPs. Surprisingly, the abundance of $\mathrm{F}_{2}$-IsoPs is reduced in baked salmon as compared to raw fish [104]. Hence, the use of $\mathrm{F}_{2}$-IsoPs could also be an indication to the quality of lipids present. The feasibility of using $\mathrm{F}_{2}$-IsoPs as a lipid quality indicator is especially important because fatty fishes constitute a large portion of healthy dietary lipids, and there is a risk falling ill if bad quality lipids are ingested. Of note, $\mathrm{F}_{2}$-dihomo-IsoPs were also detected in the muscles of marine fish by Chung et al., but were not statistically different from normoxia and hypoxia treatments. However, hyperoxia-stressed medaka fish showed elevation of dihomo-isofurans, which are produced from AdA non-enzymatically under higher oxygen tension [105].

In environmental stressor studies, $\mathrm{F}_{2}$-IsoPs are elevated in human and animal subjects when exposed to air pollutants, such as wood smoke particles, ozone, fine particulate matter (PM2.5), black carbon, and diesel exhaust. Other environmental contaminants also include formaldehyde, organophosphate insecticides, plasticizer, and perfluoroctanesulfonic acid (also an industrial contaminant). Collectively, the exact mechanism of action by these stressors to induce lipid peroxidation remains largely unknown, but has shown to cause discomfort and other complications along with oxidative stress. Remarkably, special attention should be paid to the potential role of $15-\mathrm{F}_{2 \mathrm{t}}$-IsoP as a wastewater biomarker for the assessment of community public health 


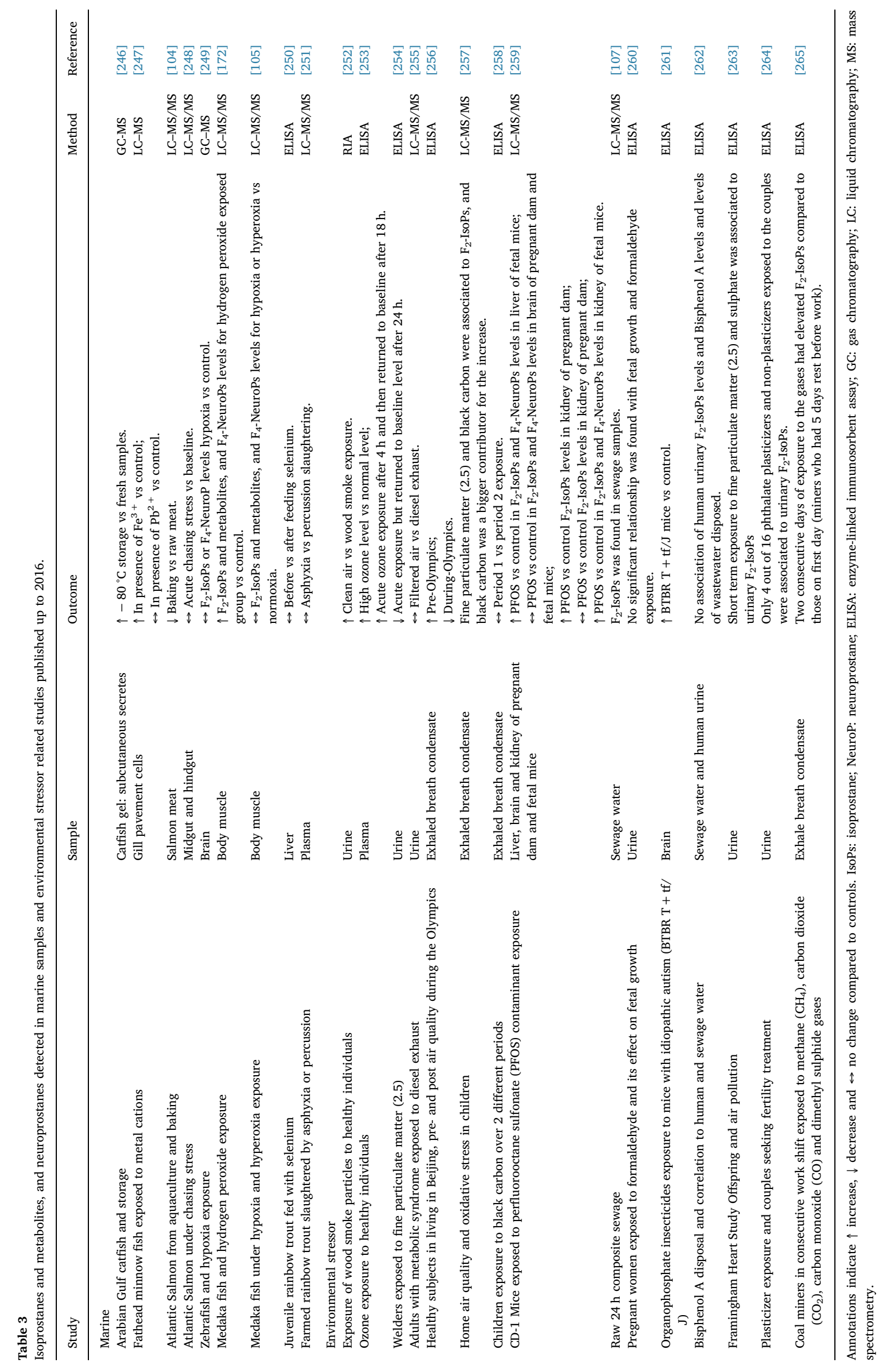


due to its high stability [106]. Recently, Ryu et al. have successfully detected $15-\mathrm{F}_{2 \mathrm{t}}$-IsoP in sewage samples [107] and found the levels to be associated with trans-3'-hydroxycotinine, a tobacco metabolite in the same samples [108]. While the concept of $15-\mathrm{F}_{2 \mathrm{t}}$-IsoP as the biomarker for community health assessment has only just been propositioned, the findings indicate tobacco smoking is habitual in the community of the sewage origin. Nevertheless, longer periods of monitoring are required to prove on its suitability as well as the stability in the sewage as it is highly exposed to microbial pathogens.

\subsection{Phytoprostanes}

PhytoPs are predominantly found in higher plants as mammals do not endogenously produce ALA due to the lack of delta-12 and delta-15 desaturases which are needed for the synthesis of essential fatty acids. Identified by Mueller and co-workers, they reported the presence of two series ( 9 and 16) of $E_{1}$-PhytoPs [19] and $F_{1}$-PhytoPs [109] from plant cultures. Other PhytoPs discovered include $\mathrm{A}_{1^{-}}, \mathrm{B}_{1^{-}}, \mathrm{D}_{1^{-}}$, and $\mathrm{L}_{1^{-}}$PhytoPs. Among which $A_{1}$ - and $B_{1}$-PhytoPs are produced in low abundance in plants, where $A_{1}$-PhytoPs are readily isomerised into $B_{1}$-PhytoPs under extreme $\mathrm{pH}$ or high temperature. $\mathrm{D}_{1}$ - and $\mathrm{F}_{1}$-PhytoPs are the most dominant PhytoPs in plants [110]. Plants constitutively produce ROS as signalling molecules for cell growth and development, but also significantly intensify PhytoP production under stress. Therefore, PhytoPs serve as an excellent biomarker for oxidative degradation of plant food. In addition, PhytoP can be esterified to galactolipid and was detected in Arabidopsis thaliana plant by Feussner's group [111].

The Gil-Izquierdo group has shown that PhytoPs of walnuts are sensitive biomarkers towards improper storage conditions (e.g. nonvacuum versus vacuum packed, and storing at $24^{\circ} \mathrm{C}$ versus $4{ }^{\circ} \mathrm{C}$ ) [112]. This suggests that the quality of plant oils could potentially be assessed by the measurement of PhytoPs. Also, Table 4 illustrates the wide variety of PhytoPs found in different plant samples, like peppermint leave, melon leave, almond cultivars, red wine, and dietary oils (e.g. sunflower, flaxseed, olive, soybean, rapeseed, walnut and grape seed oils). More importantly, olive oil being low in ALA showed the presence of $\mathrm{F}_{1}$-PhytoPs in plasma and urine after consumption, but the biological activity to date, are not fully explored. Because Mediterranean diet contains high amount of extra virgin olive oil further investigation regarding the PhytoPs bioactivities is of great importance.

\subsection{Neuroprostanes}

The formation of neuroprostanes (NeuroPs) proceeds via the nonenzymatic oxygenation of DHA. They are widely used as a specific biomarker for oxidative damage of brain tissues. Clinical and experimental results in human and animal models consistently showed that oxidative damage to the brain induces an increment of $\mathrm{F}_{4}$-NeuroPs production as depicted in Table 5. Interestingly, studies which measured different series of $\mathrm{F}_{4}$-NeuroPs showed that $10-\mathrm{F}_{4 \mathrm{t}}$-NeuroP, but rarely $4-\mathrm{F}_{4 \mathrm{t}}-\mathrm{NeuroP}$, was found in the urine samples. Probably, this implies that the excretion of $10-\mathrm{F}_{4 \mathrm{t}}$-NeuroP might be subjected to further metabolism.

In some studies, $\mathrm{A}_{4} / \mathrm{J}_{4}$-NeuroPs were similarly elevated from ROS insult. For example, rats exposed to alcohol [113] and in tick-borne encephalitis patients [114] had higher amounts of $\mathrm{A}_{4} / \mathrm{J}_{4}$-NeuroPs compared to control group brain tissue and plasma, respectively. Apparently, $\mathrm{F}_{4}$-NeuroPs appears to be a more suitable biomarker for neurodegeneration and brain injury, including the Rett Syndrome and ischemic-stroke, compared to $\mathrm{F}_{2}$-IsoPs. The advancement of quantifying $\mathrm{F}_{4}$-NeuroPs clinically for early detection of debilitating neurological disorders has been slow, mostly due to the lack of commercially available standards for human testing. Currently, only a handful of studies were conducted in neurodegenerative disease patients, and more work is required to fully understand their biological role in these diseases.
In summary, $\mathrm{F}_{4}$-NeuroPs continue to serve as a reliable biomarker for brain oxidative damages, but whether the correlation of brain $\mathrm{F}_{4-}$ NeuroPs and plasma or urine $\mathrm{F}_{4}$-NeuroPs can be established for an early detection of neurological disorder, such as neurodegenerative diseases, requires further experimental data.

\section{Bioactivities of isoprostanoids}

In addition to their application as biomarkers of oxidative stress, isoprostanoids were reported to exert biological activities. They serve as homeostatic mediators in keeping physiological functions, or involve in inflammation and immunity that are associated to the pathology of diseases. The following section reviews the current knowledge of the bioactivities of isoprostanoids, namely IsoPs, NeuroPs and PhytoPs.

\subsection{Isoprostanes}

AA derived $15-\mathrm{F}_{2 \mathrm{t}}$-IsoP was initially found to be a potent renal vasoconstrictor in the low nanomolar range [115]. In subsequent studies of $15-\mathrm{F}_{2 \mathrm{t}}$-IsoP, a similar constriction effect was observed in the vascular systems such as the liver, heart, lung [116,117], smooth muscle [2] and retina [118], in peripheral lymphatics [119] and the airways [120]. Other biological activities of $15-\mathrm{F}_{2 \mathrm{t}}$-IsoP also include inhibition of angiogenesis [121] and modulation of glutamate release from the bovine retinae [122]. The biological activities of $15-\mathrm{F}_{2 \mathrm{t}}-\mathrm{IsoP}$ are generally thought to be exerted as an agonist for the activation of thromboxane receptors (TPR), that can be abrogated by the TPR antagonist SQ29548 $[115,116,123]$. Interestingly, unlike other TPR ligands, e.g. thromboxane A2, the binding mechanism of $15-\mathrm{F}_{2 \mathrm{t}}$-IsoP to the active site of the platelet TPRs revealed a unique coordination profile as studied by site-directed mutagenesis. While $\mathrm{Phe}^{184}$ and $\mathrm{Asp}^{193}$ are shared among $15-\mathrm{F}_{2 \mathrm{t}}$-IsoP and other TPR ligands, the binding of $15-\mathrm{F}_{2 \mathrm{t}}$-IsoP to TPRs requires an additional interaction with $\mathrm{Phe}^{196}$ [124]. In the same study, the second $15-\mathrm{F}_{2 \mathrm{t}}$-IsoP binding site on platelet was identified which constitutes to a total opposite effect. This was realised via an unknown cAMP-coupled receptor that inhibits the activation of platelet [124]. Therefore, the biological activities of $15-\mathrm{F}_{2 \mathrm{t}}$-IsoP can be platelet activating or inhibiting, depending on of the active sites that is occupied. Nonetheless, not all $\mathrm{F}_{2}$-IsoPs are homogenous in their bioactivity and mode of actions. For example, $15-\mathrm{F}_{2 \mathrm{c}}$-IsoP activates the $\mathrm{PGF}_{2 \alpha}$ receptor at high concentration and induces hypertrophy of cardiomyocytes via intracellular signalling pathways different from those of $\mathrm{PGF}_{2 \alpha}[125,126]$. Also, 5- $\mathrm{F}_{2 \mathrm{t}}$-IsoP, another abundantly generated $\mathrm{F}_{2^{-}}$ IsoP isomer analogous to $15-\mathrm{F}_{2 \mathrm{t}}$-IsoP, showed no vasomotor activity $[127,128]$. Exploration into the bioactivity of 12 - and 8-series $\mathrm{F}_{2}$-IsoPs has been limited largely due to the low abundance in vivo, except for one study that revealed $12-\mathrm{F}_{2 \mathrm{t}}$-IsoP and its epimer, $12-$ epi- $12-\mathrm{F}_{2 \mathrm{t}}$-IsoP, induced pig retinal and brain vasoconstriction in a dose-dependent manner [128].

In addition to $\mathrm{F}_{2}$-IsoPs exhibiting biological activities, arachidonic acid-derived E-ring IsoPs were widely studied. For instance, $15-\mathrm{E}_{2 \mathrm{t}}-\mathrm{IsoP}$, one of the two IsoPs (together with $15-\mathrm{F}_{2 \mathrm{t}}$-IsoP) that was available for biological testing in the early years, has shown to be a renal vasoconstrictor that caused a reduction in the glomerular capillary pressure and filtration rate at low nanomolar range [129]. This $15-\mathrm{E}_{2 \mathrm{t}}$-IsoP was even more potent than $15-\mathrm{F}_{2 \mathrm{t}}$-IsoP in activation of $\mathrm{TP}$ and $\mathrm{EP}_{3}$ receptors $[130,131]$. Furthermore, $15-\mathrm{E}_{2 \mathrm{t}}-\mathrm{Iso} \mathrm{P}$ has been reported to activate intestinal epithelial cells contraction [132], stimulate the binding of monocytes to the endothelial cells [133], exert contractile activity on gastrointestinal smooth muscle [134] and act as a regulator of the airways $[135,136]$. In contrast, the analogous $15-\mathrm{D}_{2}$-IsoP has received less attention while its chemical total synthesis has been described but being rather an instable compound [137].

While $\mathrm{D}_{2^{-}}$and $\mathrm{E}_{2}$-IsoPs are not the final metabolites of the $\mathrm{AA}$ peroxidation pathway, they are subjected to dehydration for the formation of $\mathrm{J}_{2^{-}}$and $\mathrm{A}_{2}$-IsoP, respectively. Since the depiction of $\mathrm{A}_{2^{-}}$[47] 


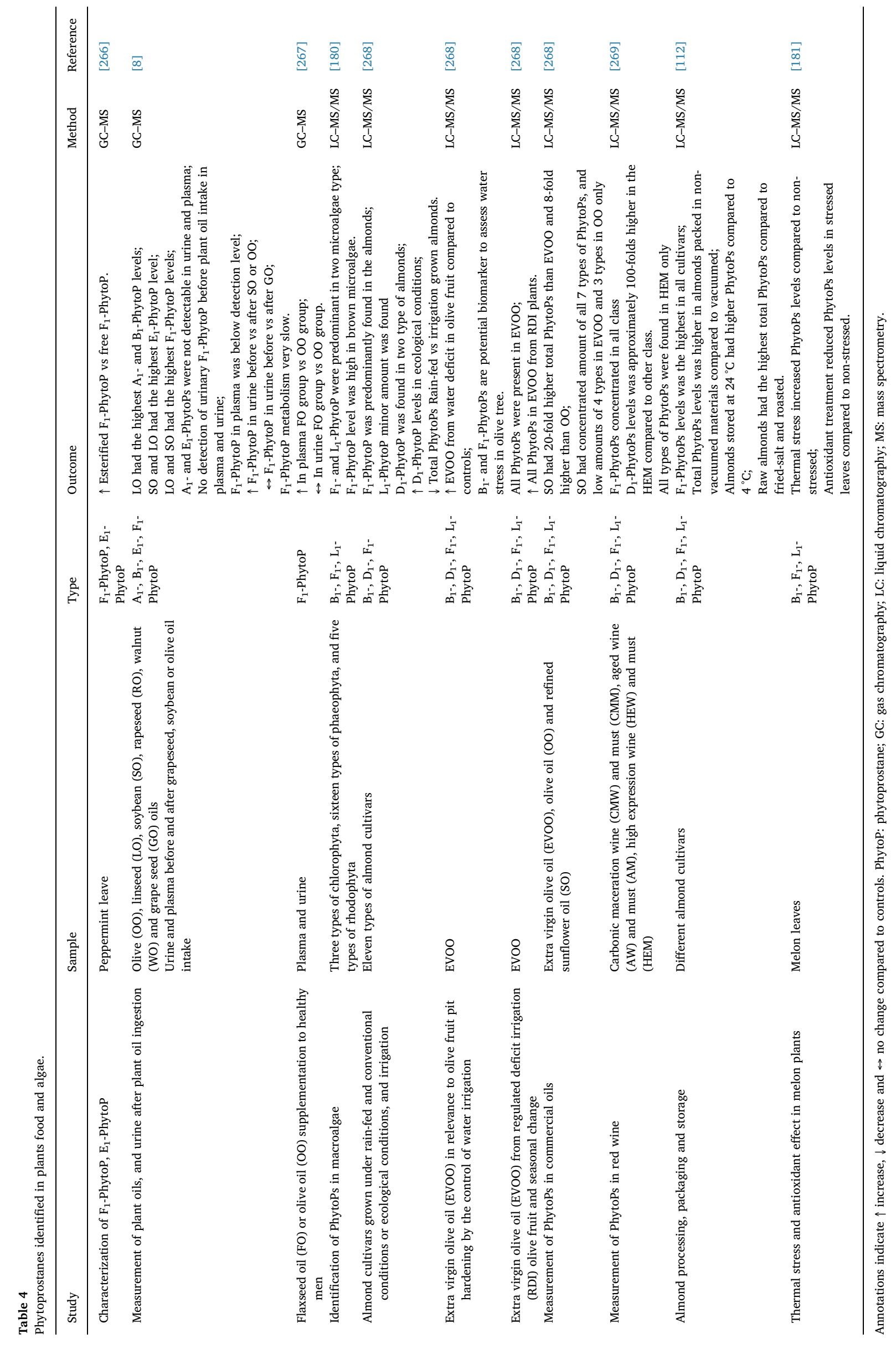


Table 5

Neuroprostanes ( $\mathrm{F}_{4}$-NeuroPs and $\mathrm{A}_{4} / \mathrm{J}_{4}$-neuroprostanes) identified and/or used as biomarkers and to indicate human disease status related to oxidative stress published up to 2016 .

\begin{tabular}{|c|c|c|c|c|}
\hline Study & Sample & Outcome & Method & Reference \\
\hline \multicolumn{5}{|l|}{ Biological studies } \\
\hline Oxidative damage to brain by kainic acid & $\begin{array}{l}\text { Rat cerebrum } \\
\text { Rat urine }\end{array}$ & $\begin{array}{l}\uparrow \text { After } 2 \mathrm{~h} \text { injection; } \\
\leftrightarrow \text { From baseline after injection. }\end{array}$ & GC-MS & [180] \\
\hline Characterization of NeuroPs using rat model & $\begin{array}{l}\text { Liver treated with } \\
\mathrm{CCl}_{4} \\
\text { Brain }\end{array}$ & $\begin{array}{l}\uparrow 4 \text { - and } 10 \text {-series NeuroPs were elevated in the } \\
\text { liver; } \\
\text { NeuroPs were identified in brain. }\end{array}$ & $\begin{array}{l}\text { LC-MS } \\
\text { GC-MS }\end{array}$ & [23] \\
\hline $\begin{array}{l}\text { Mice supplemented with fish oil (FO) and induced with or without } \\
\text { inflammation }\end{array}$ & Lung & $\begin{array}{l}\uparrow \text { FO vs control; } \\
\uparrow \text { FO + inflammation vs FO. }\end{array}$ & GC-MS & [270] \\
\hline Zebrafish under hypoxia & Brain & $\leftrightarrow$ Before vs after hypoxia. & GC-MS & [249] \\
\hline Sonoporation of Jurkat cells & Cells & $\leftrightarrow$ Sonoporated vs non-sonoporated. & LC-MS/MS & [72] \\
\hline Rats fed with alcohol with or without sweet grass (antioxidant) beverage & Brain $^{\mathrm{a}}$ & $\begin{array}{l}\uparrow \text { Alcohol vs control; } \\
\leftrightarrow \text { Sweet grass vs control; } \\
\leftrightarrow \text { Alcohol + sweet grass vs control; } \\
\downarrow \text { Alcohol + sweet grass vs alcohol. }\end{array}$ & LC-MS/MS & [113] \\
\hline $\begin{array}{l}\text { Rats liver injury by } \mathrm{CCl}_{4} \text { and supplemented with high fat diet of extra } \\
\text { virgin olive oil (EVOO) or corn oil (CO) or lard }\end{array}$ & Liver & $\begin{array}{l}\uparrow \mathrm{EVOO}, \mathrm{CO}, \text { lard vs control; } \\
\downarrow \mathrm{EVOO}+\mathrm{CCl}_{4} \text { group vs } \mathrm{CCl}_{4} \text { group; } \\
\leftrightarrow \mathrm{CO}+\mathrm{CCl}_{4} \text { or lard }+\mathrm{CCl}_{4} \text { group vs } \mathrm{CCl}_{4} \text { group. }\end{array}$ & LC-MS/MS & {$[271]$} \\
\hline \multicolumn{5}{|l|}{ Human studies } \\
\hline Healthy and smoker volunteers & Plasma & $\begin{array}{l}\uparrow \text { Smokers' vs healthy; } \\
\uparrow \text { Smokers after cigarette smoking. }\end{array}$ & GC-MS & [272] \\
\hline Healthy controls and ischemic-stroke patients & Plasma & $\uparrow$ Ischemic-stroke vs control. & GC-MS & {$[273]$} \\
\hline Type 2 diabetic patients with or without zinc supplementation & Plasma & $\begin{array}{l}\uparrow \text { Diabetic vs non-diabetic controls; } \\
\leftrightarrow \text { Diabetic placebo supplementation vs diabetic zinc } \\
\text { supplementation. }\end{array}$ & GC-MS & [220] \\
\hline Normal pregnancy and pre-eclampsia pregnancy & $\begin{array}{l}\text { Maternal and cord } \\
\text { blood plasma }\end{array}$ & $\begin{array}{l}\uparrow \text { Maternal plasma normal vs eclampsia; } \\
\uparrow \text { Cord plasma normal vs eclampsia. }\end{array}$ & GC-MS & [236] \\
\hline Female Rett syndrome patients with or without fish oil & Plasma & $\downarrow$ Before vs after fish oil supplementation. & GC-MS/MS & {$[65]$} \\
\hline Influenza A patients and healthy controls & Plasma & $\begin{array}{l}\leftrightarrow \text { Acute illness vs control; } \\
\downarrow \text { Acute illness vs } 3 \text { months post illness. }\end{array}$ & GC-MS & [239] \\
\hline $\begin{array}{l}\text { Mild Alzheimer's disease (AD), amnestic mild cognitive impairment (a- } \\
\text { MCI) and healthy control }\end{array}$ & CSF & $\begin{array}{l}\leftrightarrow \text { AD or a-MCI vs control; } \\
\leftrightarrow \text { AD vs a-MCI. }\end{array}$ & GC-MS & [184] \\
\hline $\begin{array}{l}\text { Female patients with } M E C P 2 \text { gene and Rett syndrome patients with } \\
\text { typical presentation, supplemented with or without fish oil }\end{array}$ & Plasma & $\begin{array}{l}\uparrow \text { Rett patients vs healthy controls; } \\
\downarrow \text { Rett patients before vs after supplementation. }\end{array}$ & GC-MS/MS & [274] \\
\hline Preterm infants with bronchopulmonary dysplasia (BPD) & Urine & $\leftrightarrow$ BPD vs control throughout 4 weeks gestation. & LC-MS/MS & {$[275]$} \\
\hline Epilepsy and control & Urine & $\leftrightarrow$ Epileptic group vs control. & LC-MS/MS & [102] \\
\hline Rett syndrome patients and healthy controls & Skin fibroblast & $\uparrow$ RTT patients vs control. & GC-MS/MS & [276] \\
\hline Traumatic brain injury (TBI) and controls & CSF & $\uparrow$ TBI patients post-operation vs control. & GC-MS & [191] \\
\hline Tick-borne encephalitis (TBE) patients and healthy subjects & Plasma $^{\mathrm{a}}$ & $\uparrow$ TBE vs control. & LC-MS/MS & [114] \\
\hline Athletes in altitude training (hypoxia) & Urine & $\uparrow$ Before vs after training. & LC-MS/MS & [277] \\
\hline Neuroborreliosis (NB) patients and antibiotics treatment & Urine, plasma, CSF & $\begin{array}{l}\uparrow \text { NB vs control in CSF and plasma; } \\
\downarrow \text { Before vs after in NB CSF and plasma; } \\
\text { Urine levels were not detectable. }\end{array}$ & LC-MS/MS & [200] \\
\hline Down syndromes and controls & Plasma & $\uparrow$ Down syndrome vs control. & GC-MS/MS & [278] \\
\hline Healthy females before and after wine intake & Urine & $\begin{array}{l}\downarrow \text { Before vs after and postulated to be due to } \\
\text { phenolic compound, hydroxytyrosol. }\end{array}$ & LC-MS/MS & [279] \\
\hline Epilepsy and new generation drug treatment & Urine & $\begin{array}{l}\leftrightarrow \text { Epileptic patient vs control; } \\
\uparrow \text { Epileptic patient or control vs epileptic patients } \\
\text { with new-generation antiepileptic drugs. }\end{array}$ & LC-MS/MS & [280] \\
\hline $\begin{array}{l}\text { MECP2 Duplication Syndrome (MDSS), Rett syndrome patients and } \\
\text { control }\end{array}$ & Plasma & $\begin{array}{l}\uparrow \text { MDS or Rett vs Control; } \\
\leftrightarrow \text { MDS vs Rett. }\end{array}$ & GC-MS/MS & [281] \\
\hline
\end{tabular}

${ }^{\text {a }}$ Annotation indicates $\mathrm{A}_{4} / \mathrm{J}_{4}$-neuroprostanes, otherwise it is $\mathrm{F}_{4}$-neuroprostanes. Arrows indicate $\uparrow$ increase, $\downarrow$ decrease and $\leftrightarrow$ no change compared to controls. PhytoP: phytoprostane; GC: gas chromatography; LC: liquid chromatography; MS: mass spectrometry.

and $\mathrm{J}_{2}$-IsoPs [48], their total syntheses and biological activities were investigated extensively. For example, Musiek et al. reported that 15$\mathrm{A}_{2 \mathrm{t}}$-IsoP induced caspase- 3 cleavage, leading to apoptosis in the cortical cultures at sub-micromolar concentrations [138]. Conversely, anti-inflammatory effects of $15-\mathrm{A}_{2}$-IsoPs were also reported by inhibition of the NF- $\mathrm{BB}$ pathway in lipopolysaccharide (LPS)-induced macrophages [139] and human gestational tissues [140]. Further, it was mentioned that $15-\mathrm{J}_{2}$-IsoPs, but not $15-\mathrm{A}_{2}$-IsoPs, inhibited inflammatory response via the PPAR $\gamma$ activation and induced RAW264.7 cells apoptosis in a PPAR $\gamma$-independent manner [139].

In comparison to $15-\mathrm{F}_{2 \mathrm{t}}$-IsoPs, EPA-derived IsoPs were discovered only about a decade ago and rendered as one of the least studied IsoPs. Although 5- and 18-series $\mathrm{F}_{3 \mathrm{t}}$-IsoPs are theoretically predominant over other regioisomers [141], no accounts on the bioactivity of $18-\mathrm{F}_{3 \mathrm{t}}$-IsoPs have been reported. The $5-\mathrm{F}_{3 \mathrm{t}}$-IsoPs was able to modulate the release of neurotransmitters in isolated bovine retina [142]. One report on the bioactivity of $\mathrm{F}_{3}$-IsoPs by Brooks et al. showed that $15-\mathrm{A}_{3 \mathrm{t}}$-IsoP has antiinflammatory effects on LPS-stimulated macrophages via the inhibition of NF-KB pathways, and inhibitory effect on the formation of foam cells - a major step in the pathogenesis of atherosclerosis [143]. Moreover, Gao et al. reported that $15-\mathrm{J}_{3}$-IsoPs induced Nrf2 expression by inhibiting its negative regulator Keap1 [144].

\subsection{Phytoprostanes}

Although the primary source of PhytoPs comes from plants, this section reviews their biological activities when exposed to humans. Uptake of PhytoPs in humans is predominantly through ALA-enriched diets because we do not endogenously synthesize ALA. High levels of $\mathrm{F}_{1^{-}}, \mathrm{E}_{1^{-}}, \mathrm{A}_{1^{-}}$and $\mathrm{B}_{1}$-PhytoPs have been found in vegetable oils with the concentration ranging from 0.09 to $99 \mathrm{mg} / \mathrm{L}$ [8]. In the same study, Karg et al. reported that $\mathrm{A}_{1}$-PhytoPs, and $\mathrm{B}_{1}$-PhytoPs to a lesser extent, 
inhibited dose-dependently NF- $\mathrm{BB}$ transactivation in transfected HEK 293 and LPS-activated RAW264.7 cells [8]. Also, it was reported that $\mathrm{A}_{1}$-PhytoPs, but not $\mathrm{B}_{1}$-PhytoPs, induced apoptosis in Jurkat T-cells [8]. An alternative exposure to PhytoPs is through the inhalation of pollen, of which $\mathrm{E}_{1}$-PhytoPs are the most abundant. Studies on human dendritic cells showed that $\mathrm{E}_{1}$-PhytoPs inhibited LPS-induced interleukin (IL)-12 production and enhanced T-helper 2 cell polarization [145] via a PPAR $\gamma$-dependent mechanism [146]. Recently, the study of PhytoPs has been extended to cells from the central nervous system. Minghetti and co-workers demonstrated $\mathrm{B}_{1}$-PhytoPs to be neuroprotective for undifferentiated, but not differentiated, SH-SY5Y cells from $\mathrm{H}_{2} \mathrm{O}_{2}$ insult, and promoted oligodendrocyte differentiation partly via PPAR $\gamma$ activation [9]. To date, despite the availability of $F_{1}$-PhytoPs chemical standards [51], information about their biological activity in mammals is still missing, and they remain biomarkers for oxidative damage to plants.

\subsection{Neuroprostanes}

Within the regioisomers of NeuroPs, the 4- and 20-series NeuroPs are the most abundant form of NeuroPs while, the other type can be further oxidized. Some of the NeuroP compounds and internal standards were successfully synthesized by the groups of Vidari-Zanoni [147], Taber [148] and Durand [149-151]. Remarkably, elevation of $\mathrm{F}_{4}$-NeuroPs was detected in the brain tissue of patients with neurodegenerative diseases like Alzheimer's diseases (AD) and hence it has been recognised as a reliable biomarker for neural oxidative damage. The first study on the bioactivity of NeuroP used $14-\mathrm{A}_{4}$-NeuroPs by Musiek and colleagues described $14-\mathrm{A}_{4}$-NeuroPs as a potent anti-inflammatory mediator, inhibiting NF- $\mathrm{KB}$ activation in LPS-induced macrophages [152]. Similarly, Majkova et al. showed that $\mathrm{A}_{4} / \mathrm{J}_{4}$-NeuroPs, prepared from DHA, to down-regulate PCB77-induced monocyte chemo-attractant protein-1 expression and Nrf2 activation in primary pulmonary endothelial cells [153]. It is worth noting that IsoPs/PhytoPs/NeuroPs with A- or J-ring structure displayed anti-inflammatory effects, primarily via the inhibition of NF- $\mathrm{kB}$ pathways with or without the activation of $\operatorname{PPAR} \gamma$, whereas F-ring isoprostanoids consistently failed to exhibit any beneficial effects in these models. Nevertheless, $4(R S)-4-\mathrm{F}_{4 \mathrm{t}^{-}}$ NeuroP has recently displayed cardiac anti-arrhythmic properties in vitro and in vivo via the protection of the ryanodine receptor [6]. In another follow up study by the same group, $4(R S)-4-\mathrm{F}_{4 \mathrm{t}}$-NeuroP has shown to protect ischemia-reperfusion damages by down-regulating the release of cytochrome c and caspase 3 activity [7]. In addition, 4(RS)-4$\mathrm{F}_{4 \mathrm{t}}$-NeuroP exerted anti-proliferative effects in the human breast cancer cell line (MDA-MB-231), and even more potently when encapsulated in

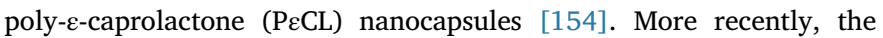
anti-inflammatory effects of $4(R S)-4-\mathrm{F}_{4 \mathrm{t}}$-NeuroP and $14-\mathrm{A}_{4 \mathrm{t}}$-NeuroP were compared with the well-known enzymatically derived anti-inflammatory lipid mediators NDP1 and PDX. The result suggested cyclopentenone NeuroPs or F-type NeuroP i.e. 4(RS)-4- $\mathrm{F}_{4 \mathrm{t}}-\mathrm{NeuroP}$ and 14$\mathrm{A}_{4 \mathrm{t}}$-NeuroP possess anti-inflammatory activities similar to the protectins in human macrophages [155]. Consequently, 4(RS)-4- $\mathrm{F}_{4 \mathrm{t}^{-}}$ NeuroP is the very first F-ring isoprostanoid compound to show bioactivities in mammalian cells, this could potentially be extrapolated into other cell types and species. This gives rise to the question whether the elevated amount of $\mathrm{F}_{4}$-NeuroPs in $\mathrm{AD}$ patients is the cause or the consequence of neurodegenerative diseases, and if these $\mathrm{F}_{4}$-NeuroPs play a role in diseases regulation.

\section{Outlook and conclusions}

Insofar, isoprostanoids have generally been used as biomarkers of oxidative damage in mammalian diseases or for plant injuries. Only for $15-\mathrm{F}_{2 \mathrm{t}}$-IsoP, metabolism and excretion has been described. However, the use of isoprostanoids as oxidative stress biomarkers in general has suffered largely from the misconception that a single isomer is suitable to reflect oxidative damage. Obviously, different cells and tissues have different PUFA composition and content. In turn, specific isoprostanoids should serve as tissue and cell specific oxidative stress markers. For example, $15-\mathrm{F}_{2 \mathrm{t}}$-IsoP might reflect blood related oxidative injury of peripheral cells and tissue, while the $4-\mathrm{F}_{4 \mathrm{t}}$-NeuroPs needs to be investigated when concerning neurological conditions. Furthermore, it was shown that isoprostanoids originating from n-3 PUFA have biological activities. Although more research needs to be conducted, protective mechanisms from muscle injuries to cancer have been described. Frequently the limitation for more detailed studies is the lack of pure compounds. This also limits the validation of analytical methods, where poor availability of the unique isoprostanoids restricts quantitative measurements. However, with the precursor and transition ions published here, and mass spectrometry spectra for LC-MS/MS, at least one can undertake qualitative evaluation of the isoprostanoids.

No doubt, there are much needs to understand about the role of isoprostanoids in marine biology. Despite being abundant in PUFA, little is known about isoprostanoids and their physiological and biological presence in marine organisms. Further, due to climate change and environmental pollution, isoprostanoids are probably released in PUFA rich marine species; whether they are detrimental or beneficial in reproduction and development is unknown.

Further, using the appropriate isoprostanoids, large human population studies need to be conducted using mass spectrometry based analysis to warrant specificity, ultimately allowing a final assessment of the usefulness of the isoprostanoids as specific oxidative damage markers [156]. Additionally, isoprostanoids such as dihomo-IsoPs and NeuroPs may be characteristic for white and grey matter damage respectively possibly presenting diseases specific biomarkers.

This review presents a comprehensive overview of isoprostanoid chemistry and biology over the past three decades hopefully helping the community to understand the role of these non-enzymatic PUFA derivatives in health and disease.

\section{Acknowledgments}

JCYL would like to thank Small Project Funding (201409176019), The University of Hong Kong for the support of this work.

\section{Appendix A. Supplementary data}

Supplementary data to this article can be found online at https:// doi.org/10.1016/j.plipres.2017.09.004.

\section{References}

[1] J.D. Morrow, K.E. Hill, R.F. Burk, T.M. Nammour, K.F. Badr, L.J. Roberts II, A series of prostaglandin F2-like compounds are produced in vivo in humans by a non-cyclooxygenase, free radical-catalyzed mechanism, Proc Natl Acad Sci U S A 87 (1990) 9383-9387.

[2] M. Fukunaga, N. Makita, L.J. Roberts II, J.D. Morrow, K. Takahashi, K.F. Badr, Evidence for the existence of F2-isoprostane receptors on rat vascular smooth muscle cells, Am J Phys 264 (1993) C1619-24.

[3] J.D. Morrow, L.J. Roberts, The isoprostanes: unique bioactive products of lipid peroxidation, Prog Lipid Res 36 (1997) 1-21.

[4] C. Chiabrando, A. Valagussa, C. Rivalta, T. Durand, A. Guy, E. Zuccato, et al., Identification and measurement of endogenous beta-oxidation metabolites of 8epi-Prostaglandin F2alpha, J Biol Chem 274 (1999) 1313-1319.

[5] B. Halliwell, C.Y. Lee, Using isoprostanes as biomarkers of oxidative stress: some rarely considered issues, Antioxid Redox Signal 13 (2010) 145-156.

[6] J. Roy, C. Oger, J. Thireau, J. Roussel, O. Mercier-Touzet, D. Faure, et al., Nonenzymatic lipid mediators, neuroprostanes, exert the antiarrhythmic properties of docosahexaenoic acid, Free Radic Biol Med 86 (2015) 269-278.

[7] J. Roy, J. Fauconnier, C. Oger, C. Farah, C. Angebault-Prouteau, J. Thireau, et al, Non-enzymatic oxidized metabolite of DHA, 4(RS)-4-F4t-neuroprostane protects the heart against reperfusion injury, Free Radic Biol Med 102 (2017) 229-239.

[8] K. Karg, V.M. Dirsch, A.M. Vollmar, J.L. Cracowski, F. Laporte, M.J. Mueller, Biologically active oxidized lipids (phytoprostanes) in the plant diet and parenteral lipid nutrition, Free Radic Res 41 (2007) 25-37.

[9] L. Minghetti, R. Salvi, M. Lavinia Salvatori, M.A. Ajmone-Cat, C. De Nuccio, S. Visentin, et al., Nonenzymatic oxygenated metabolites of alpha-linolenic acid B1- and L1-phytoprostanes protect immature neurons from oxidant injury and promote differentiation of oligodendrocyte progenitors through PPAR-gamma 
activation, Free Radic Biol Med 73 (2014) 41-50.

[10] H. Yin, N.A. Porter, J.D. Morrow, Separation and identification of F2-isoprostane regioisomers and diastereomers by novel liquid chromatographic/mass spectrometric methods, J Chromatogr B Anal Technol Biomed Life Sci 827 (2005) 157-164.

[11] J.E. Baldwin, Rules for ring closure, J Chem Soc Chem Commun (1976) 734-736.

[12] J.E. Baldwin, R.C. Thomas, L.I. Kruse, L. Silberman, Rules for ring closure: ring formation by conjugate addition of oxygen nucleophiles, J Organ Chem 42 (1977) 3846-3852.

[13] T.J. Montine, K.S. Montine, E.E. Reich, E.S. Terry, N.A. Porter, J.D. Morrow, Antioxidants significantly affect the formation of different classes of isoprostanes and neuroprostanes in rat cerebral synaptosomes, Biochem Pharmacol 65 (2003) 611-617.

[14] A.D. Watson, G. Subbanagounder, D.S. Welsbie, K.F. Faull, M. Navab, M.E. Jung, et al., Structural identification of a novel pro-inflammatory epoxyisoprostane phospholipid in mildly oxidized low density lipoprotein, J Biol Chem 274 (1999) 24787-24798.

[15] J.A. Berliner, N. Leitinger, S. Tsimikas, The role of oxidized phospholipids in atherosclerosis, J Lipid Res 50 (Suppl) (2009) S207-12.

[16] P. Bretscher, J. Egger, A. Shamshiev, M. Trotzmuller, H. Kofeler, E.M. Carreira, et al., Phospholipid oxidation generates potent anti-inflammatory lipid mediators that mimic structurally related pro-resolving eicosanoids by activating Nrf2, EMBO Mol Med 7 (2015) 593-607.

[17] R.J. Waugh, R.C. Murphy, Mass spectrometric analysis of four regioisomers of F2isoprostanes formed by free radical oxidation of arachidonic acid, J Am Soc Mass Spectrom 7 (1996) 490-499.

[18] R.J. Waugh, J.D. Morrow, L.J. Roberts II, R.C. Murphy, Identification and relative quantitation of F2-isoprostane regioisomers formed in vivo in the rat, Free Radic Biol Med 23 (1997) 943-954.

[19] S. Parchmann, M.J. Mueller, Evidence for the formation of dinor isoprostanes E1 from alpha-linolenic acid in plants, J Biol Chem 273 (1998) 32650-32655.

[20] J.A. Lawson, S. Kim, W.S. Powell, G.A. FitzGerald, J. Rokach, Oxidized derivatives of omega-3 fatty acids: identification of IPF3 alpha-VI in human urine, J Lipid Res 47 (2006) 2515-2524.

[21] J. Nourooz-Zadeh, E.H. Liu, E. Anggard, B. Halliwell, F4-isoprostanes: a novel class of prostanoids formed during peroxidation of docosahexaenoic acid (DHA), Biochem Biophys Res Commun 242 (1998) 338-344.

[22] L.J. Roberts II, T.J. Montine, W.R. Markesbery, A.R. Tapper, P. Hardy, S. Chemtob, et al., Formation of isoprostane-like compounds (neuroprostanes) in vivo from docosahexaenoic acid, J Biol Chem 273 (1998) 13605-13612.

[23] H. Yin, E.S. Musiek, L. Gao, N.A. Porter, J.D. Morrow, Regiochemistry of neuroprostanes generated from the peroxidation of docosahexaenoic acid in vitro and in vivo, J Biol Chem 280 (2005) 26600-26611.

[24] M. VanRollins, R.L. Woltjer, H. Yin, J.D. Morrow, T.J. Montine, F2-dihomo-isoprostanes arise from free radical attack on adrenic acid, J Lipid Res 49 (2008) 995-1005.

[25] U. Jahn, J.M. Galano, T. Durand, Beyond prostaglandins — chemistry and biology of cyclic oxygenated metabolites formed by free-radical pathways from polyunsaturated fatty acids, Angew Chem 47 (2008) 5894-5955.

[26] E. Jahn, T. Durand, J.-M. Galano, U. Jahn, Recent approaches to the total synthesis of phytoprostanes, isoprostanes and neuroprostanes as important products of lipid oxidative stress and biomarkers of disease, Chem List 108 (2014) 301-319.

[27] D.F. Taber, R.S. Hoerrner, Enantioselective rhodium-mediated synthesis of (-)-PGE2 methyl ester, J Organ Chem 57 (1992) 441-447.

[28] D.F. Taber, R.J. Herr, D.M. Gleave, Diastereoselective Synthesis of an Isoprostane: (+/-)-8-epi-PGF(2)(alpha) Ethyl Ester, J Organ Chem 62 (1997) 194-198.

[29] D.F. Taber, K. Kanai, Synthesis of the four enantiomerically-pure isomers of 15 F2t-isoprostane, Tetrahedron 54 (1998) 11767-11782.

[30] D.F. Taber, K. Kanai, A synthetic approach to 15-D2c-isoprostane ethyl ester, J Organ Chem 63 (1998) 6607-6609.

[31] D.F. Taber, K. Kanai, Synthesis of 2, 3-Dinor-5, 6-dihydro-15F2t-isoprostane, J Organ Chem 64 (1999) 7983-7987.

[32] D.F. Taber, Q. Jiang, Total synthesis of the four enantiomerically pure diastereomers of 8-F(2t)-isoprostane, J Organ Chem 66 (2001) 1876-1884.

[33] D.F. Taber, D. Teng, Total synthesis of the ethyl ester of the major urinary metabolite of prostaglandin E(2), J Organ Chem 67 (2002) 1607-1612.

[34] D.F. Taber, M. Xu, J.C. Hartnett, Synthesis of the eight enantiomerically pure diastereomers of the 12-F(2)-isoprostanes, J Am Chem Soc 124 (2002) $13121-13126$

[35] D.F. Taber, K. Kanai, R. Pina, 5-F2t-isoprostane, a human hormone? J Am Chem Soc 121 (1999) 7773-7777.

[36] A. Guy, T. Durand, J.-P. Vidal, J.-C. Rossi, Total synthesis of 15 (RS)-5, 6-dehydro8-epi-PGF $2 \alpha$ methyl ester by a biomimetic process, Tetrahedron Lett 38 (1997) 1543-1546.

[37] T. Durand, A. Guy, J.P. Vidal, J.C. Rossi, Total synthesis of (15R)- and (15S)-F(2t)isoprostanes by a biomimetic process using the cyclization of acyclic dihydroxylated octa-5,7-dienyl radicals, J Organ Chem 67 (2002) 3615-3624.

[38] U. Jahn, P. Hartmann, I. Dix, P.G. Jones, Oxidative enolate cyclizations of 6, 8nonadienoates: towards the synthesis of prostanes, Eur J Org Chem 2002 (2002) 718-735.

[39] U. Jahn, E. Dinca, Total synthesis of 15-F2t-isoprostane by using a new oxidative cyclization of distonic radical anions as the key step, Chemistry 15 (2009) 58-62.

[40] J. Egger, P. Bretscher, S. Freigang, M. Kopf, E.M. Carreira, Synthesis of epoxyisoprostanes: effects in reducing secretion of pro-inflammatory cytokines IL-6 and IL-12, Angew Chem 52 (2013) 5382-5385.

[41] J. Weng, S. Wang, L.J. Huang, Z.Y. Luo, G. Lu, Stereoselective synthesis of epoxyisoprostanes: an organocatalytic and "pot-economy" approach, Chem Commun (Camb) 51 (2015) 10170-10173.

[42] R.C. Larock, N.H. Lee, Organopalladium approaches to prostaglandins. 11. Synthesis of PGF2. alpha. and 12-epi PGF2. alpha. by the controlled, one step, palladium-promoted, intermolecular coupling of three different alkenes, J Am
Chem Soc 113 (1991) 7815-7816.

[43] S.W. Hwang, M. Adiyaman, S. Khanapure, L. Schio, J. Rokach, Total Synthesis of 8-epi-PGF2. alpha. A novel strategy for the synthesis of isoprostanes, J Am Chem Soc 116 (1994) 10829-10830.

[44] Z. Pudukulathan, S. Manna, S.-W. Hwang, S.P. Khanapure, J.A. Lawson, G.A. FitzGerald, et al., Diels - Alder approach to isoprostanes. total synthesis of iPF2 $\alpha-V$, J Am Chem Soc 120 (1998) 11953-11961.

[45] J. Rokach, The isoprostanes, a new class of natural products: synthesis and biosynthesis, Synthesis 1998 (1998) 569-580.

[46] S.H. Jacobo, C.T. Chang, G.J. Lee, J.A. Lawson, W.S. Powell, D. Pratico, et al., Total synthesis of 8,12-iso-iPF3alpha-VI, an EPA-derived isoprostane: stereoselective introduction of the fifth asymmetric center, J Organ Chem 71 (2006) 1370-1379.

[47] G. Zanoni, A. Porta, G. Vidari, First total synthesis of A(2) isoprostane, J Organ Chem 67 (2002) 4346-4351.

[48] G. Zanoni, A. Porta, F. Castronovo, G. Vidari, First total synthesis of JS isoprostane, J Organ Chem 68 (2003) 6005-6010.

[49] B. Rondot, T. Durand, J.P. Girard, J.C. Rossi, L. Schio, S.P. Khanapure, et al., A free radical route to syn lactones and other prostanoid intermediates in isoprostaglandin synthesis, Tetrahedron Lett 34 (1993) 8245-8248.

[50] T. Durand, A. Guy, O. Henry, A. Roland, S. Bernad, S. El Fangour, et al., Total syntheses of iso-, neuro- and phytoprostanes: new insight in lipid chemistry, Chem Phys Lipids 128 (2004) 15-33.

[51] S. El Fangour, A. Guy, V. Despres, J.P. Vidal, J.C. Rossi, T. Durand, Total synthesis of the eight diastereomers of the syn-anti-syn phytoprostanes F1 types I and II, J Organ Chem 69 (2004) 2498-2503.

[52] J.P. Vionnet, P. Renaud, Facile Preparation of ( \pm )-12-Epiprostaglandins from 7 Oxabicyclo [2.2. 1] hept-5-en-2-one via an all-cis-formyllactone related to Corey lactone, Helvetica Chim Acta 77 (1994) 1781-1790.

[53] T.O. Schrader, M.L. Snapper, Ring opening metathesis approach to the isoprostanes, Tetrahedron Lett 41 (2000) 9685-9689.

[54] T.O. Schrader, M.L. Snapper, Stereodivergent synthesis of all 15-F(2) isoprostanes, J Am Chem Soc 124 (2002) 10998-11000.

[55] M. Shizuka, T.O. Schrader, M.L. Snapper, Synthesis of isoprostanyl phosphatidylcholine and isoprostanyl phosphatidylethanolamine, J Organ Chem 71 (2006) 1330-1334.

[56] M. Shizuka, M.L. Snapper, Selective synthesis of ent-15-epi-F2t-isoprostane and a deuterated derivative, Synthesis 2007 (2007) 2397-2403.

[57] C. Oger, Y. Brinkmann, S. Bouazzaoui, T. Durand, J.M. Galano, Stereocontrolled access to isoprostanes via a bicyclo[3.3.0]octene framework, Org Lett 10 (2008) 5087-5090.

[58] H.P. Acharya, Y. Kobayashi, Total synthesis of 2-(5,6-epoxyisoprostane A2)phosphorylcholine and elucidation of the relative configuration of the isoprostane moiety, Angew Chem 44 (2005) 3481-3484.

[59] H.P. Acharya, Y. Kobayashi, Synthesis of phosphorylcholines possessing 5, 6-or 14, 15-epoxyisoprostane A 2 at sn-2 position, Tetrahedron Lett 46 (2005) 8435-8438.

[60] M.E. Jung, J.A. Berliner, D. Angst, D. Yue, L. Koroniak, A.D. Watson, et al., Total synthesis of the epoxy isoprostane phospholipids PEIPC and PECPC, Org Lett 7 (2005) 3933-3935.

[61] M.E. Jung, J.A. Berliner, L. Koroniak, B.G. Gugiu, A.D. Watson, Improved synthesis of the epoxy isoprostane phospholipid PEIPC and its reactivity with amines, Org Lett 10 (2008) 4207-4209.

[62] H. Kawashima, Y. Kobayashi, Synthesis of the PMB ether of 5,6-epoxyisoprostane E2 through aldol reaction of the alpha-bromocyclopentanone, Org Lett 16 (2014) 2598-2601.

[63] W. Zhong, J.R. Springstead, R. Al-Mubarak, S. Lee, R. Li, B. Emert, et al., An epoxyisoprostane is a major regulator of endothelial cell function, J Med Chem 56 (2013) 8521-8532.

[64] L.J. Roberts II, K.P. Moore, W.E. Zackert, J.A. Oates, J.D. Morrow, Identification of the major urinary metabolite of the F2-isoprostane 8-iso-prostaglandin F2alpha in humans, J Biol Chem 271 (1996) 20617-20620.

[65] C. De Felice, C. Signorini, T. Durand, L. Ciccoli, S. Leoncini, M. D'Esposito, et al. Partial rescue of Rett syndrome by omega- 3 polyunsaturated fatty acids (PUFAs) oil, Genes Nutr 7 (2012) 447-458.

[66] J.D. Morrow, T.M. Harris, L.J. Roberts 2nd., Noncyclooxygenase oxidative formation of a series of novel prostaglandins: analytical ramifications for measurement of eicosanoids, Anal Biochem 184 (1990) 1-10.

[67] G.L. Milne, Q. Dai, L.J. Roberts Ii, The isoprostanes-25 years later, Biochim Biophys Acta (BBA) Mol Cell Biol Lipids 1851 (2015) 433-445.

[68] J.-M. Galano, J.C.-Y. Lee, C. Gladine, B. Comte, J.-Y. Le Guennec, C. Oger, et al., Non-enzymatic cyclic oxygenated metabolites of adrenic, docosahexaenoic, eicosapentaenoic and $\alpha$-linolenic acids; bioactivities and potential use as biomarkers, Biochim Biophys Acta (BBA) Mol Cell Biol Lipids 1851 (2015) 446-455.

[69] G.L. Milne, H. Yin, K.D. Hardy, S.S. Davies, L.J. Roberts, Isoprostane generation and function, Chem Rev 111 (2011) 5973-5996.

[70] D. Kloos, H. Lingeman, O.A. Mayboroda, A.M. Deelder, W.M.A. Niessen, M. Giera, Analysis of biologically-active, endogenous carboxylic acids based on chromatography-mass spectrometry, TrAC Trends Anal Chem 61 (2014) 17-28.

[71] A.E. Barden, E. Mas, K.D. Croft, M. Phillips, T.A. Mori, Minimizing artifactual elevation of lipid peroxidation products (F2-isoprostanes) in plasma during col lection and storage, Anal Biochem 449 (2014) 129-131.

[72] K.S. Leung, X. Chen, W. Zhong, Yu AC, C.Y. Lee, Microbubble-mediated sonoporation amplified lipid peroxidation of Jurkat cells, Chem Phys Lipids 180 (2014) 53-60.

[73] J. Folch, M. Lees, G.H.A. Sloane Stanley, simple method for the isolation and purification of total lipides from animal tissues, J Biol Chem 226 (1957) 497-509.

[74] E.G. Bligh, W.J. Dyer, A rapid method of total lipid extraction and purification, Can J Biochem Physiol 37 (1959) 911-917.

[75] V. Matyash, G. Liebisch, T.V. Kurzchalia, A. Shevchenko, D. Schwudke, Lipid extraction by methyl-tert-butyl ether for high-throughput lipidomics, J Lipid Res 49 (2008) 1137-1146. 
[76] J.M. Proudfoot, A.E. Barden, K.D. Croft, J.-M. Galano, T. Durand, V. Bultel-Poncé, et al., F2-Isoprostanes in HDL are bound to neutral lipids and phospholipids, Free Radic Res 50 (2016) 1374-1385.

[77] C.Y. Lee, S.H. Huang, A.M. Jenner, B. Halliwell, Measurement of F2-isoprostanes, hydroxyeicosatetraenoic products, and oxysterols from a single plasma sample, Free Radic Biol Med 44 (2008) 1314-1322.

[78] M.L. Langhorst, M.J. Hastings, W.H. Yokoyama, S.C. Hung, N. Cellar, K. Kuppannan, et al., Determination of F2-isoprostanes in urine by online solid phase extraction coupled to liquid chromatography with tandem mass spectrometry, J Agric Food Chem 58 (2010) 6614-6620.

[79] K.S. Leung, J.M. Galano, T. Durand, J.C. Lee, Current development in non-enzymatic lipid peroxidation products, isoprostanoids and isofuranoids, in novel biological samples, Free Radic Res 49 (2015) 816-826.

[80] J.H. Dahl, R.B. van Breemen, Rapid quantitative analysis of 8-iso-prostaglandinF2á using liquid chromatography-tandem mass spectrometry and comparison with an enzyme immunoassay method, Anal Biochem 404 (2010) 211-216.

[81] G.L. Milne, S.C. Sanchez, E.S. Musiek, J.D. Morrow, Quantification of F2-isoprostanes as a biomarker of oxidative stress, Nat Protoc 2 (2007) 221-226.

[82] W. Liu, J.D. Morrow, H. Yin, Quantification of F2-isoprostanes as a reliable index of oxidative stress in vivo using gas chromatography-mass spectrometry (GC-MS) method, Free Radic Biol Med 47 (2009) 1101-1107.

[83] D. Tsikas, E. Schwedhelm, M.T. Suchy, J. Niemann, F.M. Gutzki, V.J. Erpenbeck, et al., Divergence in urinary 8-iso-PGF(2alpha) (iPF(2alpha)-III, 15-F(2t)-IsoP) levels from gas chromatography-tandem mass spectrometry quantification after thin-layer chromatography and immunoaffinity column chromatography reveals heterogeneity of 8-iso-PGF(2alpha). Possible methodological, mechanistic and clinical implications, J Chromatogr B Anal Technol Biomed Life Sci 794 (2003) 237-255.

[84] D. Tsikas, A.A. Zoerner, Analysis of eicosanoids by LC-MS/MS and GC-MS/MS: A historical retrospect and a discussion, J Chromatogr B 964 (2014) 79-88.

[85] J.D. Morrow, L.J. Roberts II, Mass spectrometry of prostanoids: F2-isoprostanes produced by non-cyclooxygenase free radical-catalyzed mechanism, Methods Enzymol 233 (1994) 163-174.

[86] J.G. Bollinger, G.S. Naika, M. Sadilek, M.H.L.C. Gelb, ESI-MS/MS detection of FAs by charge reversal derivatization with more than four orders of magnitude improvement in sensitivity, J Lipid Res 54 (2013) 3523-3530.

[87] A. Kretschmer, M. Giera, M. Wijtmans, L. de Vries, H. Lingeman, H. Irth, et al., Derivatization of carboxylic acids with 4-APEBA for detection by positive-ion LCESI-MS(/MS) applied for the analysis of prostanoids and NSAID in urine, J Chromatogr B 879 (2011) 1393-1401.

[88] Y.Y. Lee, J.M. Galano, C. Oger, C. Vigor, R. Guillaume, J. Roy, et al., Assessment of isoprostanes in human plasma: technical considerations and the use of mass spectrometry, Lipids 51 (2016) 1217-1229.

[89] D.R. Briskey, G.R. Wilson, R.G. Fassett, J.S. Coombes, Optimized method for quantification of total $\mathrm{F}(2)$-isoprostanes using gas chromatography-tandem mass spectrometry, J Pharm Biomed Anal 90 (2014) 161-166.

[90] D. Tsikas, M.-T. Suchy, Protocols for the measurement of the F2-isoprostane, 15(S)-8-iso-prostaglandin F2á, in biological samples by GC-MS or GC-MS/MS coupled with immunoaffinity column chromatography, J Chromatogr B 1019 (2016) 191-201.

[91] M. Korecka, C.M. Clark, V.M.Y. Lee, J.Q. Trojanowski, L.M. Shaw, Simultaneous HPLC-MS-MS quantification of 8-iso-PGF2á and 8,12-iso-iPF2á in CSF and brain tissue samples with on-line cleanup, J Chromatogr B 878 (2010) 2209-2216.

[92] S.A. Brose, B.T. Thuen, M.Y.L.C. Golovko, MS/MS method for analysis of E2 series prostaglandins and isoprostanes, J Lipid Res 52 (2011) 850-859.

[93] A. Dupuy, P. Le Faouder, C. Vigor, C. Oger, J.M. Galano, C. Dray, et al., Simultaneous quantitative profiling of 20 isoprostanoids from omega-3 and omega-6 polyunsaturated fatty acids by LC-MS/MS in various biological samples, Anal Chim Acta 921 (2016) 46-58.

[94] H.S. Jónasdóttir, C. Papan, S. Fabritz, L. Balas, T. Durand, I. Hardardottir, et al, Differential mobility separation of leukotrienes and protectins, Anal Chem 87 (2015) 5036-5040

[95] J. Kapron, J. Wu, T. Mauriala, P. Clark, R.W. Purves, K.P. Bateman, Simultaneous analysis of prostanoids using liquid chromatography/high-field asymmetric waveform ion mobility spectrometry/tandem mass spectrometry, Rapid Commun Mass Spectrom 20 (2006) 1504-1510.

[96] K.S. Leung, H.F. Chan, H.H. Leung, J.M. Galano, C. Oger, T. Durand, et al., Shorttime UVA exposure to human keratinocytes instigated polyunsaturated fatty acid without inducing lipid peroxidation, Free Radic Res 51 (2017) 269-280.

[97] C. Cuyamendous, K.S. Leung, V. Bultel-Poncé, A. Guy, T. Durand, J.-M. Galano, et al., Total syntheses and in vivo quantitation of novel phytofurans derived from alpha-linolenic acid, Eur J Org Chem (2017).

[98] T.J. van 't Erve, M.B. Kadiiska, S.J. London, R.P. Mason, Classifying oxidative stress by F2-isoprostane levels across human diseases: a meta-analysis, Redox Biol 12 (2017) 582-599.

[99] C.Y. Lee, R.C. Seet, S.H. Huang, L.H. Long, B. Halliwell, Different patterns of oxidized lipid products in plasma and urine of dengue fever, stroke, and Parkinson's disease patients: cautions in the use of biomarkers of oxidative stress, Antioxid Redox Signal 11 (2009) 407-420.

[100] Y. Huang, M. Zhu, Z. Li, R. Sa, Q. Chu, Q. Zhang, et al., Mass spectrometry-based metabolomic profiling identifies alterations in salivary redox status and fatty acid metabolism in response to inflammation and oxidative stress in periodontal disease, Free Radic Biol Med 70 (2014) 223-232.

[101] C. De Felice, C. Signorini, T. Durand, C. Oger, A. Guy, V. Bultel-Ponce, et al., F2dihomo-isoprostanes as potential early biomarkers of lipid oxidative damage in Rett syndrome, J Lipid Res 52 (2011) 2287-2297.

[102] S. Medina, I.D. Miguel-Elizaga, C. Oger, J.M. Galano, T. Durand, M. MartinezVillanueva, et al., Dihomo-isoprostanes-nonenzymatic metabolites of AdA-are higher in epileptic patients compared to healthy individuals by a new ultrahigh pressure liquid chromatography-triple quadrupole-tandem mass spectrometry method, Free Radic Biol Med 79 (2015) 154-163.
[103] L.A. Garcia-Flores, S. Medina, C. Oger, J.M. Galano, T. Durand, R. Cejuela, et al., Lipidomic approach in young adult triathletes: effect of supplementation with a polyphenols-rich juice on neuroprostane and F2-dihomo-isoprostane markers, Food Funct 7 (2016) 4343-4355.

[104] S.K. Raatz, M.Y. Golovko, S.A. Brose, T.A. Rosenberger, G.S. Burr, W.R. Wolters, et al., Baking reduces prostaglandin, resolvin, and hydroxy-fatty acid content of farm-raised Atlantic salmon (Salmo salar), J Agric Food Chem 59 (2011) 11278-11286.

[105] M.L. Chung, J.M. Galano, C. Oger, T. Durand, J.C. Lee, Hyperoxia elevates adrenic acid peroxidation in marine fish and is associated with reproductive pheromone mediators, Mar Drugs 13 (2015) 2215-2232.

[106] C.G. Daughton, Using biomarkers in sewage to monitor community-wide human health: isoprostanes as conceptual prototype, Sci Total Environ 424 (2012) 16-38.

[107] Y. Ryu, M.J. Reid, K.V. Thomas, Liquid chromatography-high resolution mass spectrometry with immunoaffinity clean-up for the determination of the oxidative stress biomarker 8-iso-prostaglandin F2alpha in wastewater, J Chromatogr A 1409 (2015) 146-151.

[108] Y. Ryu, E. Gracia-Lor, R. Bade, J.A. Baz-Lomba, J.G. Bramness, S. Castiglioni, et al., Increased levels of the oxidative stress biomarker 8-iso-prostaglandin F2alpha in wastewater associated with tobacco use, Sci Rep 6 (2016) 39055.

[109] R. Imbusch, M.J. Mueller, Formation of isoprostane F(2)-like compounds (phyto prostanes F(1)) from alpha-linolenic acid in plants, Free Radic Biol Med 28 (2000) 720-726.

[110] I. Thoma, M. Krischke, C. Loeffler, M.J. Mueller, The isoprostanoid pathway in plants, Chem Phys Lipids 128 (2004) 135-148.

[111] A. Ibrahim, A.L. Schutz, J.M. Galano, C. Herrfurth, K. Feussner, T. Durand, et al., The alphabet of galactolipids in arabidopsis thaliana, Front Plant Sci 2 (2011) 95.

[112] A.M. Carrasco-Del Amor, E. Aguayo, J. Collado-Gonzalez, A. Guy, J.M. Galano, T. Durand, et al., Impact of packaging atmosphere, storage and processing conditions on the generation of phytoprostanes as quality processing compounds in almond kernels, Food Chem 211 (2016) 869-875.

[113] W. Luczaj, I. Jarocka-Karpowicz, K. Bielawska, E. Skrzydlewska, Sweet grass protection against oxidative stress formation in the rat brain, Metab Brain Dis 30 (2015) 183-190.

[114] W. Luczaj, A. Moniuszko, I. Jarocka-Karpowicz, S. Pancewicz, L. Andrisic, N. Zarkovic, et al., Tick-borne encephalitis-lipid peroxidation and its consequences, Scand J Clin Lab Invest 76 (2016) 1-9.

[115] K. Takahashi, T.M. Nammour, M. Fukunaga, J. Ebert, J.D. Morrow, L.J. Roberts 2ndet al., Glomerular actions of a free radical-generated novel prostaglandin, 8epi-prostaglandin F2 alpha, in the rat. Evidence for interaction with thromboxane A2 receptors, J Clin Invest 90 (1992) 136-141.

[116] M. Banerjee, K.H. Kang, J.D. Morrow, L.J. Roberts, J.H. Newman, Effects of a novel prostaglandin, 8-epi-PGF2 alpha, in rabbit lung in situ, Am J Phys 263 (1992) H660-3.

[117] K.H. Kang, J.D. Morrow, L.J. Roberts 2nd, J.H. Newman, M. Banerjee, Airway and vascular effects of 8-epi-prostaglandin F2 alpha in isolated perfused rat lung, J Appl Physiol (1985) 74 (1993) 460-465.

[118] I. Lahaie, P. Hardy, X. Hou, H. Hassessian, P. Asselin, P. Lachapelle, et al., A novel mechanism for vasoconstrictor action of 8-isoprostaglandin F2 alpha on retinal vessels, Am J Phys 274 (1998) R1406-16.

[119] H. Sinzinger, A. Oguogho, J. Kaliman, Isoprostane 8-epi-prostaglandin F2 alpha is a potent contractor of human peripheral lymphatics, Lymphology 30 (1997) 155-159.

[120] A. Okazawa, I. Kawikova, Z.H. Cui, B.E. Skoogh, J. Lotvall, 8-Epi-PGF2alpha induces airflow obstruction and airway plasma exudation in vivo, Am J Respir Crit Care Med 155 (1997) 436-441.

[121] R.A. Benndorf, E. Schwedhelm, A. Gnann, R. Taheri, G. Kom, M. Didie, et al., Isoprostanes inhibit vascular endothelial growth factor-induced endothelial cell migration, tube formation, and cardiac vessel sprouting in vitro, as well as angiogenesis in vivo via activation of the thromboxane $\mathrm{A}(2)$ receptor: a potential link between oxidative stress and impaired angiogenesis, Circ Res 103 (2008) 1037-1046.

[122] C.A. Opere, W.D. Zheng, J. Huang, A. Adewale, M. Kruglet, S.E. Ohia, Dual effect of isoprostanes on the release of [3H]D-aspartate from isolated bovine retinae: role of arachidonic acid metabolites, Neurochem Res 30 (2005) 129-137.

[123] J.D. Morrow, T.A. Minton, L.J. Roberts 2nd, The F2-isoprostane, 8-epi-prostaglandin F2 alpha, a potent agonist of the vascular thromboxane/endoperoxide receptor, is a platelet thromboxane/endoperoxide receptor antagonist, Prostaglandins 44 (1992) 155-163.

[124] F.T. Khasawneh, J.S. Huang, F. Mir, S. Srinivasan, C. Tiruppathi, G.C. Le Breton, Characterization of isoprostane signaling: evidence for a unique coordination profile of 8-iso-PGF(2alpha) with the thromboxane $\mathrm{A}(2)$ receptor, and activation of a separate CAMP-dependent inhibitory pathway in human platelets, Biochem Pharmacol 75 (2008) 2301-2315.

[125] P. Kunapuli, J.A. Lawson, J. Rokach, G.A. FitzGerald, Functional characterization of the ocular prostaglandin f2alpha (PGF2alpha) receptor. Activation by the isoprostane, 12-iso-PGF2alpha, J Biol Chem 272 (1997) 27147-27154.

[126] P. Kunapuli, J.A. Lawson, J.A. Rokach, J.L. Meinkoth, G.A. FitzGerald, Prostaglandin F2alpha (PGF2alpha) and the isoprostane, 8, 12-iso-isoprostane F2alpha-III, induce cardiomyocyte hypertrophy. Differential activation of downstream signaling pathways, J Biol Chem 273 (1998) 22442-22452.

[127] S. Marliere, J.L. Cracowski, T. Durand, O. Chavanon, J. Bessard, A. Guy, et al., The 5 -series $\mathrm{F}(2)$-isoprostanes possess no vasomotor effects in the rat thoracic aorta, the human internal mammary artery and the human saphenous vein, $\mathrm{Br} \mathrm{J}$ Pharmacol 135 (2002) 1276-1280.

[128] X. Hou, L.J. Roberts 2nd, F. Gobeil Jr., D. Taber, K. Kanai, D. Abran, et al., Isomerspecific contractile effects of a series of synthetic f2-isoprostanes on retinal and cerebral microvasculature, Free Radic Biol Med 36 (2004) 163-172.

[129] J.D. Morrow, T.A. Minton, C.R. Mukundan, M.D. Campbell, W.E. Zackert, V.C. Daniel, et al., Free radical-induced generation of isoprostanes in vivo. Evidence for the formation of D-ring and E-ring isoprostanes, J Biol Chem 269 
(1994) 4317-4326.

[130] L.J. Janssen, T. Tazzeo, Involvement of TP and EP3 receptors in vasoconstrictor responses to isoprostanes in pulmonary vasculature, J Pharmacol Exp Ther 301 (2002) 1060-1066.

[131] J.L. Cracowski, P. Devillier, O. Chavanon, F.A. Sietchiping-Nzepa, F. StankeLabesque, G. Bessard, Isoprostaglandin E2 type-III (8-iso-prostaglandin E2) evoked contractions in the human internal mammary artery, Life Sci 68 (2001) 2405-2413.

[132] J.L. Elmhurst, P.A. Betti, P.K. Rangachari, Intestinal effects of isoprostanes: evidence for the involvement of prostanoid EP and TP receptors, J Pharmacol Exp Ther 282 (1997) 1198-1205.

[133] J. Huber, V.N. Bochkov, B.R. Binder, N. Leitinger, The isoprostane 8-iso-PGE2 stimulates endothelial cells to bind monocytes via cyclic AMP- and p38 MAP kinase-dependent signaling pathways, Antioxid Redox Signal 5 (2003) 163-169.

[134] W. Sametz, S. Hennerbichler, S. Glaser, R. Wintersteiger, H. Juan, Characterization of prostanoid receptors mediating actions of the isoprostanes, 8-iso-PGE(2) and 8iso-PGF(2alpha), in some isolated smooth muscle preparations, Br J Pharmacol 130 (2000) 1903-1910.

[135] A. Catalli, D. Zhang, L.J. Janssen, Receptors and signaling pathway underlying relaxations to isoprostanes in canine and porcine airway smooth muscle, Am J Phys Lung Cell Mol Phys 283 (2002) L1151-9.

[136] A.P. Joy, E.A. Cowley, 8-iso-PGE2 stimulates anion efflux from airway epithelial cells via the EP4 prostanoid receptor, Am J Respir Cell Mol Biol 38 (2008) 143-152.

[137] Y. Brinkmann, C. Oger, A. Guy, T. Durand, J.M. Galano, Total synthesis of 15-D (2t)- and 15-epi-15-E(2t)-isoprostanes, J Organ Chem 75 (2010) 2411-2414.

[138] E.S. Musiek, R.S. Breeding, G.L. Milne, G. Zanoni, J.D. Morrow, B. McLaughlin, Cyclopentenone isoprostanes are novel bioactive products of lipid oxidation which enhance neurodegeneration, J Neurochem 97 (2006) 1301-1313.

[139] E.S. Musiek, L. Gao, G.L. Milne, W. Han, M.B. Everhart, D. Wang, et al., Cyclopentenone isoprostanes inhibit the inflammatory response in macrophages, $\mathrm{J}$ Biol Chem 280 (2005) 35562-35570.

[140] M. Lappas, M. Permezel, S.J. Holdsworth, G. Zanoni, A. Porta, G.E. Rice, Antiinflammatory effects of the cyclopentenone isoprostane 15-A(2)-IsoP in human gestational tissues, Free Radic Biol Med 42 (2007) 1791-1796.

[141] L. Gao, H. Yin, G.L. Milne, N.A. Porter, J.D. Morrow, Formation of F-ring isoprostane-like compounds (F3-isoprostanes) in vivo from eicosapentaenoic acid, J Biol Chem 281 (2006) 14092-14099.

[142] J. Jamil, P. Bankhele, A. Salvi, J.E. Mannix, C. Oger, A. Guy, et al., Role of the nonenzymatic metabolite of eicosapentaenoic acid, 5-epi-5-F3t-isoprostane in the regulation of [(3)H]D-aspartate release in isolated bovine retina, Neurochem Res 39 (2014) 2360-2369.

[143] J.D. Brooks, E.S. Musiek, T.R. Koestner, J.N. Stankowski, J.R. Howard, E.M. Brunoldi, et al., The fatty acid oxidation product 15-A3t-isoprostane is a potent inhibitor of NFkappaB transcription and macrophage transformation, J Neurochem 119 (2011) 604-616.

[144] L. Gao, J. Wang, K.R. Sekhar, H. Yin, N.F. Yared, S.N. Schneider, et al., Novel n-3 fatty acid oxidation products activate Nrf2 by destabilizing the association between Keap1 and Cullin3, J Biol Chem 282 (2007) 2529-2537.

[145] C. Traidl-Hoffmann, V. Mariani, H. Hochrein, K. Karg, H. Wagner, J. Ring, et al., Pollen-associated phytoprostanes inhibit dendritic cell interleukin-12 production and augment T helper type 2 cell polarization, J Exp Med 201 (2005) 627-636.

[146] S. Gilles, V. Mariani, M. Bryce, M.J. Mueller, J. Ring, T. Jakob, et al., Pollenderived E1-phytoprostanes signal via PPAR-gamma and NF-kappaB-dependent mechanisms, J Immunol 182 (2009) 6653-6658.

[147] G. Zanoni, E.M. Brunoldi, A. Porta, G. Vidari, Asymmetric synthesis of 14-A4tneuroprostane: hunting for a suitable biomarker for neurodegenerative diseases, $\mathrm{J}$ Organ Chem 72 (2007) 9698-9703.

[148] D.F. Taber, P.G. Reddy, K.O.A. Arneson, potential route to neuroprostanes and isoprostanes: preparation of the four enantiomerically pure diastereomers of 13 F4t-neuroP, J Organ Chem 73 (2008) 3467-3474.

[149] T. Durand, A. Guy, J.-P. Vidal, J. Viala, J.-C. Rossi, Total synthesis of 4(RS)-F4t isoprostane methyl ester, Tetrahedron Lett 41 (2000) 3859-3862.

[150] C. Oger, V. Bultel-Ponce, A. Guy, L. Balas, J.C. Rossi, T. Durand, et al., The handy use of Brown's P2-Ni catalyst for a skipped diyne deuteration: application to the synthesis of a [D4]-labeled F4t-neuroprostane, Chemistry 16 (2010) $13976-13980$.

[151] A. Guy, C. Oger, J. Heppekausen, C. Signorini, C. De Felice, A. Furstner, et al., Oxygenated metabolites of $\mathrm{n}-3$ polyunsaturated fatty acids as potential oxidative stress biomarkers: total synthesis of 8-F3t-IsoP, 10-F4t-NeuroP and [D4]-10-F4tNeuroP, Chemistry 20 (2014) 6374-6380.

[152] E.S. Musiek, J.D. Brooks, M. Joo, E. Brunoldi, A. Porta, G. Zanoni, et al., Electrophilic cyclopentenone neuroprostanes are anti-inflammatory mediators formed from the peroxidation of the omega- 3 polyunsaturated fatty acid docosahexaenoic acid, J Biol Chem 283 (2008) 19927-19935.

[153] Z. Majkova, J. Layne, M. Sunkara, A.J. Morris, M. Toborek, B. Hennig, Omega-3 fatty acid oxidation products prevent vascular endothelial cell activation by coplanar polychlorinated biphenyls, Toxicol Appl Pharmacol 251 (2011) 41-49.

[154] J. Roy, L.T. Oliveira, C. Oger, J.M. Galano, V. Bultel-Ponce, S. Richard, et al., Polymeric nanocapsules prevent oxidation of core-loaded molecules: evidence based on the effects of docosahexaenoic acid and neuroprostane on breast cancer cells proliferation, J Exp Clin Cancer Res 34 (2015) 155.

[155] R. Bosviel, L. Joumard-Cubizolles, G. Chinetti-Gbaguidi, D. Bayle, C. Copin, N. Hennuyer, et al., DHA-derived oxylipins, neuroprostanes and protectins, differentially and dose-dependently modulate the inflammatory response in human macrophages: putative mechanisms through PPAR activation, Free Radic Biol Med 103 (2017) 146-154.

[156] G.L. Milne, Classifying oxidative stress by F2-Isoprostane levels in human disease: the re-imagining of a biomarker, Redox Biol 12 (2017) 897-898.

[157] A. Dupuy, P. Le Faouder, C. Vigor, C. Oger, J.M. Galano, C. Dray, et al., Simultaneous quantitative profiling of 20 isoprostanoids from omega- 3 and omega-6 polyunsaturated fatty acids by LC-MS/MS in various biological samples, Anal Chim Acta 921 (2016) 46-58.

[158] J. Larose, P. Julien, J.P. Bilodeau, F2-isoprostanes analysis on plasma of pregnan women by HPLC-MS/MS using a column packed with core-shell particles, J Lipid Res 54 (2013) 1505-1511.

[159] M. Korecka, C.M. Clark, V.M. Lee, J.Q. Trojanowski, L.M. Shaw, Simultaneous HPLC-MS-MS quantification of 8-iso-PGF(2alpha) and 8,12-iso-iPF(2alpha) in CSF and brain tissue samples with on-line cleanup, J Chromatogr B Anal Technol Biomed Life Sci 878 (2010) 2209-2216.

[160] A. de la Torre, Y. Lee, C. Oger, P. Sangild, T. Durand, J. Lee, et al., Synthesis, discovery, and quantitation of dihomo-isofurans: biomarkers for in vivo adrenic acid peroxidation, Angew Chem Int Ed Eng 53 (2014) 6249-6252.

[161] T. Sicilia, A. Mally, U. Schauer, A. Pahler, W. Volkel, LC-MS/MS methods for the detection of isoprostanes (iPF2alpha-III and 8,12-iso-iPF2alpha-VI) as biomarkers of CCl4-induced oxidative damage to hepatic tissue, J Chromatogr B Anal Technol Biomed Life Sci 861 (2008) 48-55.

[162] N.E. Bastani, T.E. Gundersen, R. Blomhoff, Determination of 8-epi PGF(2alpha) concentrations as a biomarker of oxidative stress using triple-stage liquid chromatography/tandem mass spectrometry, Rapid Commun Mass Spectrom 23 (2009) 2885-2890.

[163] D.D. Shinde, K.-B. Kim, K.-S. Oh, N. Abdalla, K.-H. Liu, S.K. Bae, et al., LC-MS/MS for the simultaneous analysis of arachidonic acid and 32 related metabolites in human plasma: Basal plasma concentrations and aspirin-induced changes of eicosanoids, J Chromatogr B 911 (2012) 113-121.

[164] A.W. Taylor, R.S. Bruno, M.G. Traber, Women and smokers have elevated urinary F2-isoprostane metabolites: a novel extraction and LC-MS methodology, Lipids 43 (2008) 925-936.

[165] J.K. Prasain, A. Arabshahi, P.R. Taub, S. Sweeney, R. Moore, J.D. Sharer, et al., Simultaneous quantification of F2-isoprostanes and prostaglandins in human urine by liquid chromatography tandem-mass spectrometry, J Chromatogr B Anal Technol Biomed Life Sci 913-914 (2013) 161-168.

[166] K. Sterz, G. Scherer, J.A. Ecker, simple and robust UPLC-SRM/MS method to quantify urinary eicosanoids, J Lipid Res 53 (2012) 1026-1036.

[167] D. Pelclova, Z. Fenclova, P. KAČER, M. Kuzma, T. NAVRÁTIL, J. LEBEDOVÁ, Increased 8-isoprostane, a marker of oxidative stress in exhaled breath condensate in subjects with asbestos exposure, Ind Health 46 (2008) 484-489.

[168] M. Janicka, P. Kubica, A. Kot-Wasik, J. Kot, J. Namieśnik, Sensitive determination of isoprostanes in exhaled breath condensate samples with use of liquid chromatography-tandem mass spectrometry, J Chromatogr B 893 (2012) 144-149.

[169] C.-N. Wang, J.Y.-S. Chen, S. Sabu, Y.-L. Chang, S.-D. Chang, C.-C. Kao, et al., Elevated amniotic fluid F2-isoprostane: a potential predictive marker for preeclampsia, Free Radic Biol Med 50 (2011) 1124-1130.

[170] M.L. Chung, K.Y. Lee, C.Y. Lee, Profiling of oxidized lipid products of marine fish under acute oxidative stress, Food Chem Toxicol 53 (2013) 205-213.

[171] I. De las Heras-Gómez, S. Medina, T. Casas-Pina, L. Marín-Soler, A. Tomás, P. Martínez-Hernández, et al., Potential applications of lipid peroxidation products-F 4-neuroprostanes, F 3-neuroprostanes n-6 DPA, F 2-dihomo-isoprostanes and $\mathrm{F}$ 2-isoprostanes-in the evaluation of the allograft function in renal transplantation, Free Radical Biol Med (2017).

[172] C.F. Labuschagne, A.B. Brenkman, Current methods in quantifying ROS and oxidative damage in Caenorhabditis elegans and other model organism of aging, Ageing Res Rev (2013).

[173] A.M. Carrasco-Del Amor, E. Aguayo, J. Collado-Gonzalez, A. Guy, J.M. Galano, T. Durand, et al., Impact of processing conditions on the phytoprostanes profile of three types of nut kernels, Free Radic Res 51 (2017) 141-147.

[174] J. Collado-Gonzalez, S. Medina, T. Durand, A. Guy, J.M. Galano, A. Torrecillas et al., New UHPLC-QqQ-MS/MS method for quantitative and qualitative determination of free phytoprostanes in foodstuffs of commercial olive and sunflower oils, Food Chem 178 (2015) 212-220.

[175] S. Medina, J. Collado-Gonzalez, F. Ferreres, J. Londono-Londono, C. JimenezCartagena, A. Guy, et al., Quantification of phytoprostanes - bioactive oxylipins and phenolic compounds of Passiflora edulis Sims shell using UHPLC-QqQ-MS/MS and LC-IT-DAD-MS/MS, Food Chem 229 (2017) 1-8.

[176] M. Barbosa, J. Collado-Gonzalez, P.B. Andrade, F. Ferreres, P. Valentao, J.M. Galano, et al., Nonenzymatic alpha-linolenic acid derivatives from the sea: macroalgae as novel sources of phytoprostanes, J Agric Food Chem 63 (2015) 6466-6474.

[177] M.E. Yonny, A. Rodriguez Torresi, C. Cuyamendous, G. Reversat, C. Oger, J.M. Galano, et al., Thermal stress in melon plants: phytoprostanes and phytofurans as oxidative stress biomarkers and the effect of antioxidant supplementation, J Agric Food Chem 64 (2016) 8296-8304.

[178] D. Pratico, J. Rokach, R.K. Tangirala, Brains of aged apolipoprotein E-deficient mice have increased levels of F2-isoprostanes, in vivo markers of lipid peroxidation, J Neurochem 73 (1999) 736-741.

[179] T.J. Montine, L. Shinobu, K.S. Montine, L.J. Roberts 2nd, N.W. Kowall, M.F. Beal, et al., No difference in plasma or urinary F2-isoprostanes among patients with Huntington's disease or Alzheimer's disease and controls, Ann Neurol 48 (2000) 950.

[180] T.J. Montine, J.F. Quinn, D. Milatovic, L.C. Silbert, T. Dang, S. Sanchez, et al., Peripheral F2-isoprostanes and F4-neuroprostanes are not increased in Alzheimer's disease, Ann Neurol 52 (2002) 175-179.

[181] D. Pratico, C.M. Clark, F. Liun, J. Rokach, V.Y. Lee, J.Q. Trojanowski, Increase of brain oxidative stress in mild cognitive impairment: a possible predictor of Alzheimer disease, Arch Neurol 59 (2002) 972-976.

[182] J.P. Fessel, C. Hulette, S. Powell, L.J. Roberts 2nd, J. Zhang, Isofurans, but not F2isoprostanes, are increased in the substantia nigra of patients with Parkinson's disease and with dementia with Lewy body disease, J Neurochem 85 (2003) 645-650.

[183] M.C. Irizarry, Y. Yao, B.T. Hyman, J.H. Growdon, D. Pratico, Plasma F2A isoprostane levels in Alzheimer's and Parkinson's disease, Neurodegener Dis 4 (2007) 403-405. 
[184] H.C. Kuo, H.C. Yen, C.C. Huang, W.C. Hsu, H.J. Wei, C.L. Lin, Cerebrospinal fluid biomarkers for neuropsychological symptoms in early stage of late-onset Alzheimer's disease, Int J Neurosci 125 (2015) 747-754.

[185] D. Pratico, V. MYL, J.Q. Trojanowski, J. Rokach, G.A. Fitzgerald, Increased F2isoprostanes in Alzheimer's disease: evidence for enhanced lipid peroxidation in vivo, FASEB J 12 (1998) 1777-1783.

[186] S.K. Abbott, A.M. Jenner, A.S. Spiro, M. Batterham, G.M. Halliday, B. Garner, Fatty acid composition of the anterior cingulate cortex indicates a high susceptibility to lipid peroxidation in Parkinson's disease, J Parkinsons Dis 5 (2015) 175-185.

[187] E.E. Lee, L.T. Eyler, O.M. Wolkowitz, A.S. Martin, C. Reuter, H. Kraemer, et al., Elevated plasma F2-isoprostane levels in schizophrenia, Schizophr Res 176 (2016) 320-326.

[188] N.C. Ward, K.D. Croft, D. Blacker, G.J. Hankey, A. Barden, T.A. Mori, et al., Cytochrome P450 metabolites of arachidonic acid are elevated in stroke patients compared with healthy controls, Clin Sci (Lond) 121 (2011) 501-507.

[189] I. Zitnanova, P. Siarnik, B. Kollar, M. Chomova, P. Pazderova, L. Andrezalova, et al., Oxidative stress markers and their dynamic changes in patients after acute ischemic stroke, Oxidative Med Cell Longev 2016 (2016) 9761697.

[190] T.B. Corcoran, E. Mas, A.E. Barden, T. Durand, J.M. Galano, L.J. Roberts, et al., Are isofurans and neuroprostanes increased after subarachnoid hemorrhage and traumatic brain injury? Antioxid Redox Signal 15 (2011) 2663-2667.

[191] H.C. Yen, T.W. Chen, T.C. Yang, H.J. Wei, J.C. Hsu, C.L. Lin, Levels of F2-isoprostanes, F4-neuroprostanes, and total nitrate/nitrite in plasma and cerebrospinal fluid of patients with traumatic brain injury, Free Radic Res 49 (2015) 1419-1430.

[192] M. Ercegovac, N. Jovic, T. Simic, L. Beslac-Bumbasirevic, D. Sokic, T. Djukic, et al., Byproducts of protein, lipid and DNA oxidative damage and antioxidant enzyme activities in seizure, Seizure 19 (2010) 205-210.

[193] S. Grosso, M. Longini, A. Rodriguez, F. Proietti, B. Piccini, P. Balestri, et al., Oxidative stress in children affected by epileptic encephalopathies, J Neurol Sci 300 (2011) 103-106

[194] A.A. Tolun, P.M. Scarbrough, H. Zhang, J.A. McKillop, F. Wang, P.S. Kishnani, et al., Systemic oxidative stress, as measured by urinary allantoin and F(2)-isoprostanes, is not increased in Down syndrome, Ann Epidemiol 22 (2012) 892-894.

[195] S.P. Chen, Y.T. Chung, T.Y. Liu, Y.F. Wang, J.L. Fuh, S.J. Wang, Oxidative stress and increased formation of vasoconstricting F2-isoprostanes in patients with reversible cerebral vasoconstriction syndrome, Free Radic Biol Med 61 (2013) 243-248.

[196] P. Gorrindo, C.J. Lane, E.B. Lee, B. McLaughlin, P. Levitt, Enrichment of elevated plasma F2t-isoprostane levels in individuals with autism who are stratified by presence of gastrointestinal dysfunction, PLoS One 8 (2013) e68444.

[197] H. Qasem, L. Al-Ayadhi, A. El-Ansary, Cysteinyl leukotriene correlated with 8 isoprostane levels as predictive biomarkers for sensory dysfunction in autism, Lipids Health Dis 15 (2016) 130.

[198] E.F. Coccaro, R. Lee, D. Gozal, Elevated plasma oxidative stress markers in individuals with intermittent explosive disorder and correlation with aggression in humans, Biol Psychiatry 79 (2016) 127-135.

[199] C. Chafer-Pericas, M. Cernada, L. Rahkonen, V. Stefanovic, S. Andersson, M. Vento, Preliminary case control study to establish the correlation between novel peroxidation biomarkers in cord serum and the severity of hypoxic ischemic encephalopathy, Free Radic Biol Med 97 (2016) 244-249.

[200] A. Moniuszko-Malinowska, W. Luczaj, I. Jarocka-Karpowicz, S. Pancewicz, J. Zajkowska, L. Andrisic, et al., Lipid peroxidation in the pathogenesis of neuroborreliosis, Free Radic Biol Med 96 (2016) 255-263.

[201] Z. Mallat, T. Nakamura, J. Ohan, G. Leseche, A. Tedgui, J. Maclouf, et al., The relationship of hydroxyeicosatetraenoic acids and F2-isoprostanes to plaque instability in human carotid atherosclerosis, J Clin Invest 103 (1999) 421-427.

[202] J.-L. Cracowski, J.-P. Baguet, O. Ormezzano, J. Bessard, F. Stanke-Labesque, G. Bessard, et al., Lipid peroxidation is not increased in patients with untreated mild-to-moderate hypertension, Hypertension 41 (2003) 286-288.

[203] N.C. Ward, J.M. Hodgson, I.B. Puddey, T.A. Mori, L.J. Beilin, K.D. Croft, Oxidative stress in human hypertension: association with antihypertensive treatment, gender, nutrition, and lifestyle, Free Radic Biol Med 36 (2004) 226-232.

[204] R. Rodrigo, H. Prat, W. Passalacqua, J. Araya, C. Guichard, J.P. Bachler, Relationship between oxidative stress and essential hypertension, Hypertens Res 30 (2007) 1159-1167.

[205] B. Dolegowska, W. Blogowski, K. Kedzierska, K. Safranow, K. Jakubowska, M. Olszewska, et al., Platelets arachidonic acid metabolism in patients with essential hypertension, Platelets 20 (2009) 242-249.

[206] S.R. Horbal, W. Seffens, A.R. Davis, N. Silvestrov, G.H. Gibbons, R.C. Quarells, et al., Associations of apelin, visfatin, and urinary 8-isoprostane with severe hypertension in African Americans: the MH-GRID study, Am J Hypertens 29 (2016) $814-820$.

[207] K.C. Tan, W.S. Chow, J.C. Lam, B. Lam, W.K. Wong, S. Tam, et al., HDL dysfunction in obstructive sleep apnea, Atherosclerosis 184 (2006) 377-382.

[208] R.V. Cherneva, Z.V. Cherneva, O.B. Georgiev, D.S. Petrova, J.I. Petrova, 8-isoprostanes and resistin as markers of vascular damage in non-hypersomnolent obstructive sleep apnoea patients, Clin Physiol Funct Imaging (2016).

[209] J.Y. Kim, Y.J. Hyun, Y. Jang, B.K. Lee, J.S. Chae, S.E. Kim, et al., Lipoproteinassociated phospholipase A2 activity is associated with coronary artery disease and markers of oxidative stress: a case-control study, Am J Clin Nutr 88 (2008) 630-637.

[210] I. Fort-Gallifa, A. Garcia-Heredia, A. Hernandez-Aguilera, J.M. Simo, J. Sepulveda, V. Martin-Paredero, et al., Biochemical indices of oxidative stress and inflammation in the evaluation of peripheral artery disease, Free Radic Biol Med 97 (2016) $568-576$.

[211] C.E. Kure, F.L. Rosenfeldt, A.B. Scholey, A. Pipingas, D.M. Kaye, P.J. Bergin, et al., Relationships among cognitive function and cerebral blood flow, oxidative stress, and inflammation in older heart failure patients, J Card Fail 22 (2016) 548-559.

[212] M. Brys, A. Morel, E. Forma, A. Krzeslak, J. Wilkosz, W. Rozanski, et al., Relationship of urinary isoprostanes to prostate cancer occurrence, Mol Cell
Biochem 372 (2013) 149-153.

[213] G.A. Asare, G. Akuffo, D. Doku, B. Asiedu, S. Santa, Dynamics of urinary oxidative stress biomarkers: 8-hydroxy-2'-deoxyguanosine and 8-isoprostane in uterine leiomyomas, J Midlife Health 7 (2016) 8-14.

[214] S. Basu, H. Harris, A. Wolk, A. Rossary, F. Caldefie-Chezet, M.P. Vasson, et al, Inflammatory F2-isoprostane, prostaglandin F2alpha, pentraxin 3 levels and breast cancer risk: the Swedish Mammography Cohort, Prostaglandins Leukot Essent Fat Acids 113 (2016) 28-32.

[215] J. Kauppi, J. Rasanen, E. Sihvo, U. Nieminen, P. Arkkila, M. Ahotupa, et al., Increased oxidative stress in the proximal stomach of patients with Barrett's esophagus and adenocarcinoma of the esophagus and esophagogastric junction, Transl Oncol 9 (2016) 336-339.

[216] L.G. Wood, M.L. Garg, J.L. Simpson, T.A. Mori, K.D. Croft, P.A. Wark, et al., Induced sputum 8-isoprostane concentrations in inflammatory airway diseases, Am J Respir Crit Care Med 171 (2005) 426-430.

[217] C. Feillet-Coudray, F. Chone, F. Michel, E. Rock, P. Thieblot, Y. Rayssiguier, et al., Divergence in plasmatic and urinary isoprostane levels in type 2 diabetes, Clin Chim Acta 324 (2002) 25-30.

[218] V. Calabrese, C. Mancuso, M. Sapienza, E. Puleo, S. Calafato, C. Cornelius, et al., Oxidative stress and cellular stress response in diabetic nephropathy, Cell Stress Chaperones 12 (2007) 299-306.

[219] K. Kathir, J.M. Dennis, K.D. Croft, T.A. Mori, A.K. Lau, M.R. Adams, et al., Equivalent lipid oxidation profiles in advanced atherosclerotic lesions of carotid endarterectomy plaques obtained from symptomatic type 2 diabetic and nondiabetic subjects, Free Radic Biol Med 49 (2010) 481-486.

[220] R.C. Seet, C.Y. Lee, E.C. Lim, A.M. Quek, H. Huang, S.H. Huang, et al., Oral zinc supplementation does not improve oxidative stress or vascular function in patients with type 2 diabetes with normal zinc levels, Atherosclerosis 219 (2011) 231-239.

[221] N. Stadler, S. Heeneman, S. Voo, N. Stanley, G.I. Giles, B.P. Gang, et al., Reduced metal ion concentrations in atherosclerotic plaques from subjects with type 2 diabetes mellitus, Atherosclerosis 222 (2012) 512-518.

[222] J. Vessby, S. Basu, R. Mohsen, C. Berne, B. Vessby, Oxidative stress and antioxidant status in type 1 diabetes mellitus, J Intern Med 251 (2002) 69-76.

[223] B. Hansel, P. Giral, E. Nobecourt, S. Chantepie, E. Bruckert, M.J. Chapman, et al., Metabolic syndrome is associated with elevated oxidative stress and dysfunctional dense high-density lipoprotein particles displaying impaired antioxidative activity, J Clin Endocrinol Metab 89 (2004) 4963-4971.

[224] R.C. Seet, C.Y. Lee, E.C. Lim, A.M. Quek, S.H. Huang, C.M. Khoo, et al., Markers of oxidative damage are not elevated in otherwise healthy individuals with the metabolic syndrome, Diabetes Care 33 (2010) 1140-1142.

[225] G. Dogra, N. Ward, K.D. Croft, T.A. Mori, P.H. Barrett, S.E. Herrmann, et al., Oxidant stress in nephrotic syndrome: comparison of $\mathrm{F}(2)$-isoprostanes and plasma antioxidant potential, Nephrol Dial Transplant 16 (2001) 1626-1630.

[226] B.P. Oberg, E. McMenamin, F.L. Lucas, E. McMonagle, J. Morrow, T.A. Ikizler, et al., Increased prevalence of oxidant stress and inflammation in patients with moderate to severe chronic kidney disease, Kidney Int 65 (2004) 1009-1016.

[227] L.F. Ramos, A. Shintani, T.A. Ikizler, J. Himmelfarb, Oxidative stress and inflammation are associated with adiposity in moderate to severe CKD, J Am Soc Nephrol 19 (2008) 593-599.

[228] J.L. Gamboa, FTt Billings, M.T. Bojanowski, L.A. Gilliam, C. Yu, B. Roshanravan, et al., Mitochondrial dysfunction and oxidative stress in patients with chronic kidney disease, Phys Rep 4 (2016).

[229] S. Carrillo-Ibarra, J.I. Cerrillos-Gutierrez, A. Escalante-Nunez, E. Rojas-Campos, B. Gomez-Navarro, S. Sifuentes-Franco, et al., The oxidative and inflammatory state in patients with acute renal graft dysfunction treated with tacrolimus, Oxidative Med Cell Longev 2016 (2016) 5405847.

[230] A. Aboutwerat, P.W. Pemberton, A. Smith, P.C. Burrows, R.F. McMahon, S.K. Jain, et al., Oxidant stress is a significant feature of primary biliary cirrhosis, Biochim Biophys Acta 1637 (2003) 142-150.

[231] P.W. Pemberton, A. Aboutwerat, A. Smith, P.C. Burrows, R.F. McMahon, T.W. Warnes, Oxidant stress in type I autoimmune hepatitis: the link between necroinflammation and fibrogenesis? Biochim Biophys Acta 1689 (2004) 182-189.

[232] P.W. Pemberton, A. Smith, T.W. Warnes, Non-invasive monitoring of oxidant stress in alcoholic liver disease, Scand J Gastroenterol 40 (2005) 1102-1108.

[233] J. Raszeja-Wyszomirska, K. Safranow, M. Milkiewicz, P. Milkiewicz, A. Szynkowska, E. Stachowska, Lipidic last breath of life in patients with alcoholic liver disease, Prostaglandins Other Lipid Mediat 99 (2012) 51-56.

[234] C. Matayatsuk, C.Y. Lee, R.W. Kalpravidh, P. Sirankapracha, P. Wilairat, S. Fucharoen, et al., Elevated F2-isoprostanes in thalassemic patients, Free Radic Biol Med 43 (2007) 1649-1655.

[235] C.N. Wang, J.Y. Chen, S. Sabu, Y.L. Chang, S.D. Chang, C.C. Kao, et al., Elevated amniotic fluid $\mathrm{F}(2)$-isoprostane: a potential predictive marker for preeclampsia, Free Radic Biol Med 50 (2011) 1124-1130.

[236] A.E. Barden, T.B. Corcoran, E. Mas, T. Durand, J.M. Galano, L.J. Roberts, et al., Is there a role for isofurans and neuroprostanes in pre-eclampsia and normal pregnancy? Antioxid Redox Signal 16 (2012) 165-169.

[237] J.F. Bilodeau, S. Qin Wei, J. Larose, K. Greffard, V. Moisan, F. Audibert, et al., Plasma F2-isoprostane class VI isomers at 12-18 weeks of pregnancy are associated with later occurrence of preeclampsia, Free Radic Biol Med 85 (2015) 282-287.

[238] L.B. Ware, J.P. Fessel, A.K. May, L.J. Roberts 2nd, Plasma biomarkers of oxidant stress and development of organ failure in severe sepsis, Shock 36 (2011) 12-17.

[239] M.P. Ng, J.C. Lee, W.M. Loke, L.L. Yeo, A.M. Quek, E.C. Lim, et al., Does influenza A infection increase oxidative damage? Antioxid Redox Signal 21 (2014) 1025-1031.

[240] M. Amirchaghmaghi, S.I. Hashemy, B. Alirezaei, F. Jahed Keyhani, S. Kargozar, S. Vasigh, et al., Evaluation of plasma isoprostane in patients with oral lichen planus, J Dent (Shiraz) 17 (2016) 21-25.

[241] S. Lasocki, P. Piednoir, C. Couffignal, E. Rineau, G. Dufour, T. Lefebvre, et al., Does IV iron induce plasma oxidative stress in critically ill patients? A comparison with healthy volunteers, Crit Care Med 44 (2016) 521-530. 
[242] L. Loffredo, R. Cangemi, L. Perri, E. Catasca, C. Calvieri, R. Carnevale, et al., Impaired flow-mediated dilation in hospitalized patients with community-acquired pneumonia, Eur J Intern Med 36 (2016) 74-80.

[243] W. Luczaj, E. Gindzienska-Sieskiewicz, I. Jarocka-Karpowicz, L. Andrisic, S. Sierakowski, N. Zarkovic, et al., The onset of lipid peroxidation in rheumatoid arthritis: consequences and monitoring, Free Radic Res 50 (2016) 304-313.

[244] V. Molina, B. von Dessauer, R. Rodrigo, C. Carvajal, Oxidative stress biomarkers in pediatric sepsis: a prospective observational pilot study, Redox Rep (2016) 1-8.

[245] Z. Yildirim, B. Bozkurt, D. Ozol, F. Armutcu, R. Akgedik, H. Karamanli, et al, Increased exhaled 8-isoprostane and interleukin-6 in patients with Helicobacter pylori infection, Helicobacter 21 (2016) 389-394.

[246] J.M. Al-Hassan, M. Ali, M. Thomson, C.R. Pace-Asciak, Detection of 8-epi prostaglandin F2alpha in an extract of epidermal secretion of the catfish from the Arabian Gulf, Prostaglandins Leukot Essent Fat Acids 59 (1998) 325-328.

[247] E.G. Spokas, S. Harshman, G.M. Cohen, C. Jiang, J.M. Levine, A.R. Rodriguez, et al., Release of the lipid peroxidation marker 8-epi-prostaglandin F2 alpha from isolated gill pavement cells, Environ Toxicol Chem 27 (2008) 1569-1575.

[248] R.E. Olsen, A. Svardal, T. Eide, A. Wargelius, Stress and expression of cyclooxygenases (cox1, cox2a, cox2b) and intestinal eicosanoids, in Atlantic salmon, Salmo salar L, Fish Physiol Biochem 38 (2012) 951-962.

[249] S.H. Moussavi Nik, K. Croft, T.A. Mori, M. Lardelli, The comparison of methods for measuring oxidative stress in zebrafish brains, Zebrafish 11 (2014) 248-254.

[250] R. Knight, V.L. Marlatt, J.A. Baker, B.P. Lo, A.M. deBruyn, J.R. Elphick, et al, Dietary selenium disrupts hepatic triglyceride stores and transcriptional networks associated with growth and Notch signaling in juvenile rainbow trout, Aquat Toxicol 180 (2016) 103-114.

[251] G. Secci, G. Parisi, G. Dasilva, I. Medina, Stress during slaughter increases lipid metabolites and decreases oxidative stability of farmed rainbow trout (Oncorhynchus mykiss) during frozen storage, Food Chem 190 (2016) 5-11.

[252] L. Barregard, G. Sallsten, P. Gustafson, L. Andersson, L. Johansson, S. Basu, et al., Experimental exposure to wood-smoke particles in healthy humans: effects on markers of inflammation, coagulation, and lipid peroxidation, Inhal Toxicol 18 (2006) 845-853.

[253] C. Chen, M. Arjomandi, J. Balmes, I. Tager, N. Holland, Effects of chronic and acute ozone exposure on lipid peroxidation and antioxidant capacity in healthy young adults, Environ Health Perspect 115 (2007) 1732-1737.

[254] A.M. Nuernberg, P.D. Boyce, J.M. Cavallari, S.C. Fang, E.A. Eisen, D.C. Christiani, Urinary 8-isoprostane and 8-OHdG concentrations in boilermakers with welding exposure, J Occup Environ Med 50 (2008) 182-189.

[255] J. Allen, C.A. Trenga, A. Peretz, J.H. Sullivan, C.C. Carlsten, J.D. Kaufman, Effect of diesel exhaust inhalation on antioxidant and oxidative stress responses in adults with metabolic syndrome, Inhal Toxicol 21 (2009) 1061-1067.

[256] W. Huang, G. Wang, Lu SE, H. Kipen, Y. Wang, M. Hu, et al., Inflammatory and oxidative stress responses of healthy young adults to changes in air quality during the Beijing Olympics, Am J Respir Crit Care Med 186 (2012) 1150-1159.

[257] G. Rosa, F. Majorin, S. Boisson, C. Barstow, M. Johnson, M. Kirby, et al., Assessing the impact of water filters and improved cook stoves on drinking water quality and household air pollution: a randomised controlled trial in Rwanda, PLoS One 9 (2014) e91011.

[258] S. De Prins, E. Dons, M. Van Poppel, L. Int Panis, F. Van de Mieroop, V. Nelen, et al., Airway oxidative stress and inflammation markers in exhaled breath from children are linked with exposure to black carbon, Environ Int 73 (2014) 440-446.

[259] Y.Y. Lee, C.K. Wong, C. Oger, T. Durand, J.M. Galano, J.C. Lee, Prenatal exposure to the contaminant perfluorooctane sulfonate elevates lipid peroxidation during mouse fetal development but not in the pregnant dam, Free Radic Res 49 (2015) 1015-1025.

[260] A. Amiri, A. Turner-Henson, The roles of formaldehyde exposure and oxidative stress in fetal growth in the second trimester, J Obstet Gynecol Neonatal Nurs 46 (2017) 51-62.

[261] A. De Felice, A. Greco, G. Calamandrei, L. Minghetti, Prenatal exposure to the organophosphate insecticide chlorpyrifos enhances brain oxidative stress and prostaglandin E2 synthesis in a mouse model of idiopathic autism, $\mathrm{J}$ Neuroinflammation 13 (2016) 149.

[262] J.M. Santos, D.A. Putt, M. Jurban, A. Joiakim, K. Friedrich, H. Kim, et al., levels in sewage wastewater effluents from metro Detroit communities, Environ Monit Assess 188 (2016) 585.
[263] W. Li, E.H. Wilker, K.S. Dorans, M.B. Rice, J. Schwartz, B.A. Coull, et al., Shortterm exposure to air pollution and biomarkers of oxidative stress: the Framingham Heart Study, J Am Heart Assoc 5 (2016).

[264] H. Wu, A. Olmsted, D.E. Cantonwine, S. Shahsavari, T. Rahil, C. Sites, et al., Urinary phthalate and phthalate alternative metabolites and isoprostane among couples undergoing fertility treatment, Environ Res 153 (2017) 1-7.

[265] Z. Zimet, M. Bilban, M. Marc Malovrh, P. Korosec, B. Poljsak, J. Osredkar, et al., 8 isoprostane as oxidative stress marker in coal mine workers, Biomed Environ Sci 29 (2016) 589-593.

[266] R. Imbusch, M.J. Mueller, Analysis of oxidative stress and wound-inducible dinor isoprostanes F(1) (phytoprostanes F(1)) in plants, Plant Physiol 124 (2000) 1293-1304.

[267] A.E. Barden, K.D. Croft, T. Durand, A. Guy, M.J. Mueller, T.A. Mori, Flaxseed oil supplementation increases plasma F1-phytoprostanes in healthy men, J Nutr 139 (2009) 1890-1895.

[268] A. Carrasco-Del Amor, J. Collado-Gonzalez, E. Aguayo, A. Guy, J. Galano, T. Durand, et al., Phytoprostanes in almonds: identification, quantification, and impact of cultivar and type of cultivation, RSC Adv 5 (2015) 51233-51241.

[269] J. Marhuenda, S. Medina, A. Diaz-Castro, P. Martinez-Hernandez, S. Arina, P. Zafrilla, et al., Dependency of phytoprostane fingerprints of must and wine on viticulture and enological processes, J Agric Food Chem 63 (2015) 9022-9028.

[270] H. Yin, W. Liu, K. Goleniewska, N.A. Porter, J.D. Morrow, R.S. Peebles Jr., Dietary supplementation of omega-3 fatty acid-containing fish oil suppresses F2-isoprostanes but enhances inflammatory cytokine response in a mouse model of ovalbumin-induced allergic lung inflammation, Free Radic Biol Med 47 (2009) 622-628.

[271] Y.Y. Lee, C. Crauste, H. Wang, H.H. Leung, J. Vercauteren, J.M. Galano, et al., Extra virgin olive oil reduced polyunsaturated fatty acid and cholesterol oxidation in rodent liver: Is this accounted by hydroxytyrosol - fatty acid conjugation? Chem Res Toxicol (2016).

[272] R.C. Seet, C.Y. Lee, W.M. Loke, S.H. Huang, H. Huang, W.F. Looi, et al, Biomarkers of oxidative damage in cigarette smokers: which biomarkers might reflect acute versus chronic oxidative stress? Free Radic Biol Med 50 (2011) 1787-1793.

[273] R.C. Seet, C.Y. Lee, B.P. Chan, V.K. Sharma, H.L. Teoh, N. Venketasubramanian, et al., Oxidative damage in ischemic stroke revealed using multiple biomarkers, Stroke 42 (2011) 2326-2329.

[274] C. Signorini, C. De Felice, S. Leoncini, T. Durand, J.M. Galano, A. Cortelazzo, et al., Altered erythrocyte membrane fatty acid profile in typical Rett syndrome: effects of omega-3 polyunsaturated fatty acid supplementation, Prostaglandins Leukot Essent Fat Acids 91 (2014) 183-193.

[275] J. Kuligowski, M. Aguar, D. Rook, I. Lliso, I. Torres-Cuevas, J. Escobar, et al., Urinary lipid peroxidation byproducts: are they relevant for predicting neonata morbidity in preterm infants? Antioxid Redox Signal 23 (2015) 178-184.

[276] C. Signorini, S. Leoncini, C. De Felice, A. Pecorelli, I. Meloni, F. Ariani, et al., Redox imbalance and morphological changes in skin fibroblasts in typical Rett syndrome, Oxidative Med Cell Longev 2014 (2014) 195935.

[277] L.A. Garcia-Flores, S. Medina, R. Cejuela, J.M. Martinez-Sanz, C. Oger J.M. Galano, et al., Assessment of oxidative stress biomarkers - neuroprostanes and dihomo-isoprostanes - in the urine of elite triathletes after two weeks of moderatealtitude training, Free Radic Res 50 (2016) 485-494.

[278] C. Manna, A. Officioso, F. Trojsi, G. Tedeschi, S. Leoncini, C. Signorini, et al. Increased non-protein bound iron in Down syndrome: contribution to lipid peroxidation and cognitive decline, Free Radic Res 50 (2016) 1422-1431.

[279] J. Marhuenda, S. Medina, P. Martinez-Hernandez, S. Arina, P. Zafrilla, J. Mulero, et al., Melatonin and hydroxytyrosol-rich wines influence the generation of DNA oxidation catabolites linked to mutagenesis after the ingestion of three types of wine by healthy volunteers, Food Funct 7 (2016) 4781-4796.

[280] S. Medina, R. Carrasco-Torres, M.I. Amor, C. Oger, J.-M. Galano, T. Durand, et al., Antiepileptic drugs affect lipid oxidative markers-neuroprostanes and F 2-dihomoisoprostanes-in patients with epilepsy: differences among first-, second-, and thirdgeneration drugs by UHPLC-QqQ-MS/MS, RSC Adv 6 (2016) 82969-82976.

[281] C. Signorini, C. De Felice, S. Leoncini, R.S. Moller, G. Zollo, S. Buoni, et al, MECP2 duplication syndrome: evidence of enhanced oxidative stress. a comparison with Rett syndrome, PLoS One 11 (2016) e0150101. 